\title{
Aspectos de Parametrizações para Inferências \\ Bayesianas Aproximadas para Modelos \\ de Componentes de Variância
}

\author{
Maria José Pegorin
}

Orientador: Prof. Dr. Jorge Alberto Achcar

Dissertação apresentada ao Instituto de Ciências Matemáticas de São Carlos - USP, como parte dos requisitos para a obtenção do Título de Mestre em Ciências - Área: "Ciências de Computação e Matemática Computacional".

USP - São Carlos

Agosto de 1995 
Aos meus irmãos

Aos meus pais, Pedro e

Conceição (in memorian) 


\section{Agradecimentos}

A conclusão dessa dissertação não seria possível sem o auxílio de algumas pessoas que de alguma forma contribuíram para esse projeto, às quais expresso os mais sinceros agradecimento.

Ao Prof. Dr. Jorge Alberto Achcar pela orientação sempre dedicada e pelo incentivo em todo decorrer do projeto de pesquisa.

Ao Conselho Nacional de Desenvolvimento Científico e Tecnológico (CNPq) pelo apoio financeiro.

Aos Profs. Dr. Josemar Rodrigues e Dr. Luis Milan pelas valiosas sugestões e comentários quando da realização de meu exame de qualificação.

Aos Profs. Dr. Dalton Francisco de Andrade e Dr. Luis Milan pelas valiosas sugestões e comentários quando da realização da minha defesa de Dissertação.

À Profa. Dra. Maria Creusa Bretas Salles pelas valiosas sugestões dadas no início do meu curso de Pós-Graduação.

Aos professores, funcionários, colegas de Pós-Graduação e a todos aqueles que de alguma maneira contribuíram para a realização desse trabalho, e em especial aos amigos Adriana, Carlos, Carlos (Fogo), Gaby, Josmar, Sadao, Silvana, Silvia e Vera, pela amizade, carinho e apoio durante este período, dentro e fora do ICMSC;

Especialmente a Eric F. Rodrigues pelo carinho, paciência e apoio. 


\section{Resumo}

Neste trabalho, desenvolvemos uma análise para modelos de componentes de variância. Assumindo diferentes densidades a priori para os parâmetros do modelo de componentes de variância com 2 ou 3 componentes de variância, exploramos 0 uso de métodos de aproximação de Laplace para obter as quantidades a posteriori de interesse. Também estudamos a importância de uma boa parametrização para a obtenção de resultados precisos. Além disso, também consideramos distribuições não-normais para os efeitos aleatórios e desenvolvemos um estudo comparativo considerando diferentes conjuntos de dados. 


\begin{abstract}
In this work, we develop a Bayesian analysis for variance component models. Assuming different prior densities for the parameters of the model with 2 or 3 variance components, we explore the use of Laplace approximation methods to find the posterior summaries of interest. We also study the importance of a good parametrization to get accurate results. We also consider non-normal distribution for the randon effects and we develop a comparative study considering different data sets.
\end{abstract}




\section{Índice}

\section{Capítulo 1}

Introdução

1.1 Planejamento de Classificação Hierárquica.

1.2 Formulação do Modelo de Efeitos Aleatórios com 3 componentes de Variância

1.2.1 Análise de Variância para o Modelo de Efeitos Aleatórios com 3 Componentes de Variância. .05

1.3 Modelo de Efeitos Aleatórios com 2 Componentes de Variância 08

1.3.1 Análise de Variância para o Modelo de Efeitos Aleatórios com 2 Componentes de Variância. .09

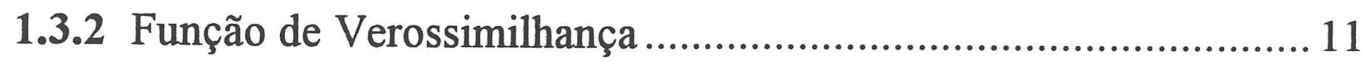

1.3.3 Estimadores de Máxima Verossimilhança................................... 12

1.4 Inferência sobre os Parâmetros $\sigma_{1}^{2}$ e $\sigma_{2}^{2}$................................................. 13

1.5 Modelo de Efeitos Aleatórios Assumindo Não-Normalidade ................ 18

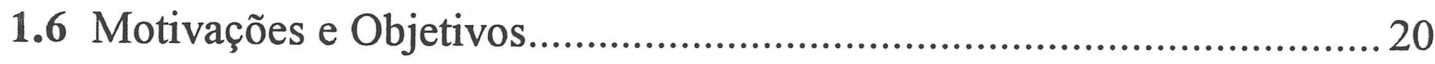

\section{Capítulo 2}

Métodos para Aproximação de Integrais.

2.1 Método de Laplace para Aproximação de Integrais

2.2 Aproximação de Laplace quando o Espaço Paramétrico Apresenta Restrições

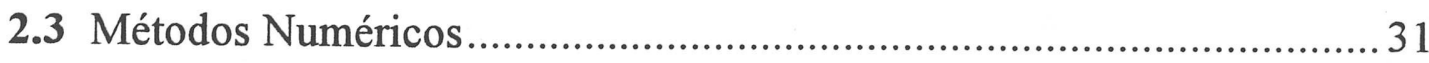

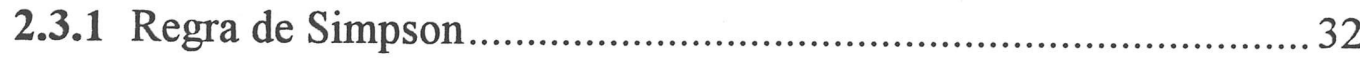

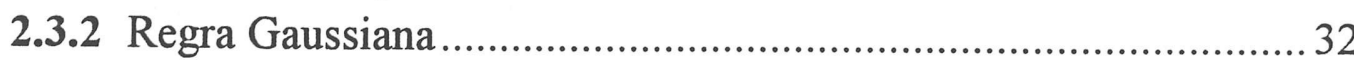

\section{Capítulo 3}

Análise Bayesiana para o Modelo de Efeitos Aleatórios com 2 Componentes de Variância

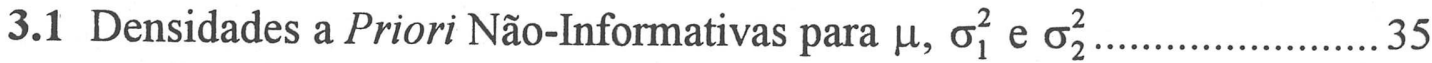

3.2 Densidades a Posteriori Conjuntas para $\mu, \sigma_{1}^{2}$ e $\sigma_{2}^{2}$ 
3.2.1 Densidades a Posteriori Marginais Conjuntas para $\sigma_{1}^{2}$ e $\sigma_{2}^{2} \ldots \ldots . .38$

3.2.2 Densidades a Posteriori Marginais Aproximadas para $\sigma_{1}^{2}$ e $\sigma_{2}^{2} \ldots 39$

3.2.3 Densidades a Posteriori Marginais para $\sigma_{2}^{2} / \sigma_{1}^{2}$....

3.3 Densidade Preditiva para a Média $Y_{J+1}$. de um Grupo Futuro................. 44

3.4 Aproximação de Laplace para Momentos a Posteriori ............................ 46

3.4.1 Aproximação de Laplace quando Ocorre o Problema de

Estimativas Clássicas Negativas................................................... 48

3.5 Fórmulas Exatas para os Momentos a Posteriori..................................51

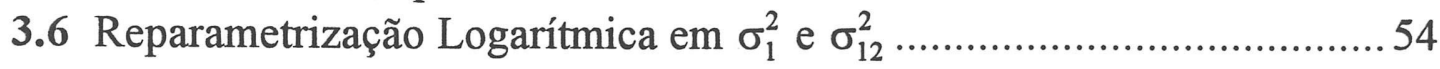

3.6.1 Aproximação de Laplace para Momentos a Posteriori

Considerando a Parametrização $\xi_{1}=\ln \left(\sigma_{1}^{2}\right)$ e

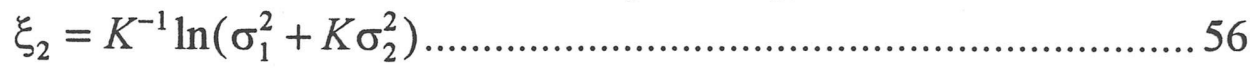

3.7 Medidas para Diagnóstico de Normalidade ...........................................58

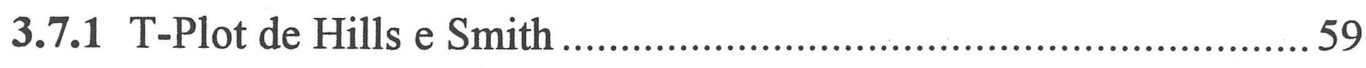

3.7.2 Terceira Derivada Padronizada .................................................6 60

\section{Capítulo 4}

Análise Bayesiana para o Modelo de Efeitos Aleatórios com 3 Componentes

de Variância

4.1 Função de Verossimilhança para o Modelo de Efeitos Aleatórios com 3 Componentes de Variância

4.2 Densidade a Priori Não-Informativa para $\mu, \sigma_{1}^{2}, \sigma_{2}^{2}$ e $\sigma_{3}^{2} \ldots \ldots \ldots \ldots \ldots \ldots \ldots . . . . . . . . .64$

4.3 Densidade a Posteriori Conjunta para $\mu, \sigma_{1}^{2}, \sigma_{2}^{2}$ e $\sigma_{3}^{2} \ldots \ldots \ldots \ldots \ldots \ldots \ldots \ldots \ldots . . . .65$

4.3.1 Densidade a Posteriori Marginal Conjunta para $\sigma_{1}^{2}, \sigma_{2}^{2}$ e $\sigma_{3}^{2} \ldots . .65$

4.3.2 Densidade a Posteriori Marginal para a Razão $\sigma_{2}^{2} / \sigma_{1}^{2} \ldots \ldots \ldots \ldots \ldots . . .66$

4.3.3 Densidade a Posteriori para Outras Funções de $\sigma_{1}^{2}, \sigma_{2}^{2}$ e $\sigma_{3}^{2} \ldots \ldots .68$

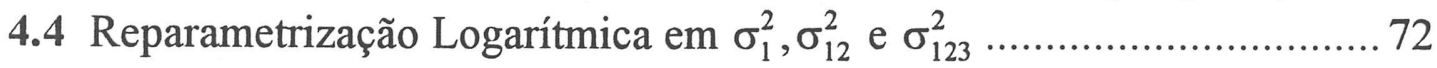

\section{Capítulo 5}

Análise Bayesiana para o Modelo de Efeitos Aleatórios Não-Normal ................... 75

5.1 Densidade a Posteriori Conjunta para $\sigma_{1}^{2}, \sigma_{1}^{2}, \phi$ e $\lambda$.............................. 76

5.2 Densidade a Posteriori Marginal Aproximada para $\phi$........................... 78

5.3 Densidade a Posteriori Marginal Aproximada para $\lambda$ com

$\phi$ Conhecido 
5.4 Uma Análise Bayesiana com $\phi$ e $\lambda$ Conhecidos 80

5.4.1 Densidade a Posteriori Marginal Aproximada para $\sigma_{1}^{2}$................ 81

5.4.2 Densidade a Posteriori Marginal Aproximada para $\sigma_{2}^{2}$ 82

5.4.3 Distribuição Preditiva Aproximada para uma Média de um Grupo Futuro $Y_{J+1}$

5.5 Uma Análise Bayesiana Assumindo $\lambda=1$ e $\mu=0$............................... 83

5.6 Densidade a Posteriori para $\theta$ 84

\section{Capítulo 6}

Exemplos de Aplicação

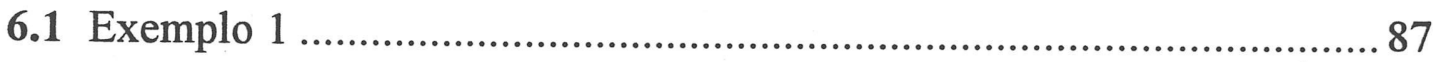

6.1.1 Análise Clássica do Problema..................................................... 88

6.1.2 Análise Bayesiana para o Exemplo 1 .........................................89

6.1.3 Comparação entre as Estimativas Obtidas.................................... 93

6.1.4 Verificação da Normalidade das Densidades a Posteriori........... 94

6.1.5 Obtenção de Momentos a Posteriori Exatos e Aproximados....... 97

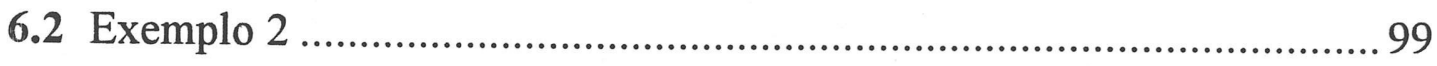

6.2.1 Análise Clássica do Problema................................................... 99

6.2.2 Análise Bayesiana para o Exemplo 2 ..................................... 100

6.2.3 Comparação entre as Estimativas Obtidas................................. 103

6.2.4 Verificação da Normalidade das Densidades a Posteriori ......... 105

6.2.5 Momentos a Posteriori Exatos e Aproximados .......................... 108

6.3 Um Exemplo Considerando o Modelo com Mistura de Normais........ 109

\section{Capítulo 7}

Conclusões e Considerações Futuras

Referências Bibliográficas.

\section{Apêndice A}

Derivadas Numéricas

\section{Apêndice B}

Obtenção de Regiões HPD 


\section{Apêndice C}

Expressões Utilizadas no Cálculo do Gráfico T-plot

Apêndice D

Alguns Programas Desenvolvidos

134 


\section{Capítulo 1}

\section{Introdução}

Um problema bastante comum num processo industrial é a existência de variabilidade nas medidas de uma característica de interesse. Geralmente os erros produzidos nessas medidas são os resultados da agregação de muitos erros.

Se a variância total da característica observada $\left(\sigma^{2}\right)$ é grande, podem ocorrer problemas relacionados ao controle da produção assim como, a obstrução da eficiência e retardamento do aperfeiçoamento do processo. Portanto, nesse caso os esforços devem ser dirigidos no sentido de reduzir $\sigma^{2}$, e para isso é necessário descobrir quais fatores estão contribuindo na variabilidade total do processo.

Para ilustrar esse problema, considere um processo químico produzindo lotes de um produto químico, o qual deve ser analisado para alguma característica de interesse. Um experimento simples consiste em retirar amostras aleatórias de cada lote e envia-las ao laboratório para serem analisadas. Desta forma, a variabilidade nos resultados refletirá uma combinação da variação da análise química, variação de amostras e variação de lotes do material.

Em outras palavras, se $\eta$ é a média verdadeira do processo e $y$ é um resultado analítico obtido, então o erro total 


$$
e=y-\eta
$$

pode ser decomposto em três componentes de erro, dados por:

1) $e_{A}$ : erro devido à análise química.

2) $e_{S}$ : erro devido à amostragem .

3) $e_{L}$ : erro devido aos lotes do produto.

Admitindo que a contribuição de cada fonte de variação (análises, amostras e lotes) ocorra de maneira aditiva, uma maneira mais simples de expressarmos o erro total (1.1) é dada por

$$
e=e_{A}+e_{S}+e_{L}
$$

Se admitimos que as variáveis $e_{A}, e_{S}$ e $e_{L}$ são independentes e com variâncias fixas $\sigma_{1}^{2}, \sigma_{2}^{2}$ e $\sigma_{3}^{2}$ respectivamente, a variância de uma medida obtida $y$, será a soma dessas três variâncias.

É importante salientar que um número menor ou maior de fontes de variação podem estar envolvidas em cada problema prático.

Usualmente, as partes que contribuem na variância total $\left(\sigma_{1}^{2}, \sigma_{2}^{2}\right.$ e $\sigma_{3}^{2}$ no exemplo) são chamadas componentes de variância (ver por exemplo, Box e Tiao 1973; ou Tiao e Tan, 1965; Tiao e Box, 1967). Descobrir como cada fonte de variação influencia na variabilidade total, implica em estimar cada componente de variância separadamente. Um experimento que torna isso possível será apresentado a seguir. 


\subsection{Planejamento de Classificação Hierárquica}

Um experimento usualmente utilizado com a finalidade de se obter estimativas dos componentes de variância é dado pelos planejamentos de classificação hierárquica (ver por exemplo, Montgomery, 1976; Box, Hunter e Hunter, 1978 ).

Esse experimento é caracterizado pelo fato de existir uma " hierarquia " entre os fatores, ou seja, os níveis de um fator são específicos à cada nível de outro fator.

Para o caso especial anterior, poderíamos considerar um planejamento de classificação hierárquica com três níveis de amostragem, o qual consistiria em selecionar aleatoriamente uma amostra de lotes, digamos $I$ lotes; para cada lote retirar $J$ amostras aleatórias de ítens e, em cada amostra, realizar repetidas análises químicas, por exemplo, $K$ análises. Na figura (1.1) verificamos a representação desse planejamento para $I=4$ lotes, $J=3$ amostras e $K=2$ análises químicas.
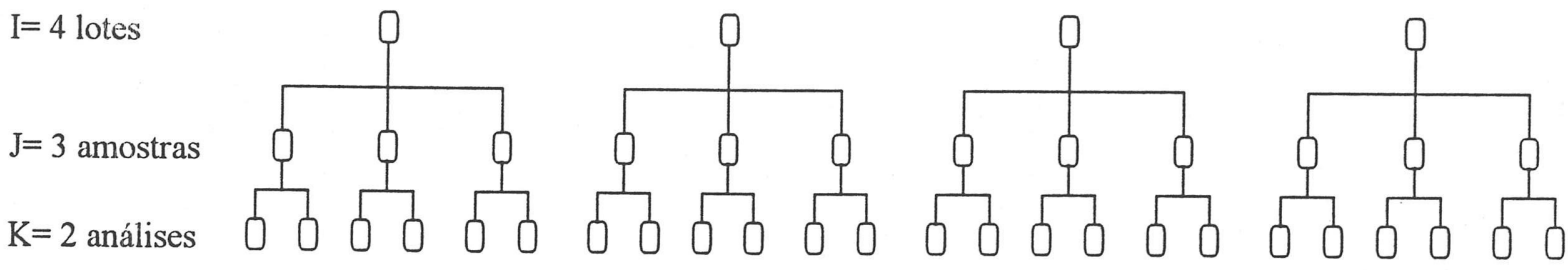

Figura 1.1 - Representação gráfica de um planejamento hierárquico com 3 níveis.

O fato do número de níveis de um fator ser igual para cada nível do outro fator torna o experimento balanceado (ver por exemplo, Montgomery, 1976).

\subsection{Formulação do Modelo de Efeitos Aleatórios com 3 Componentes de Variância}

O modelo descrito nesta seção é baseado no fato de que três fontes de variação estão envolvidas, por exemplo, lotes, amostras, e análises química. Um caso particular 
em que apenas duas fontes contribuem na variação total, será estudado com mais detalhes nas seçõ்es seguintes.

Admitindo que os erros devido a qualquer nível de amostragem contribuam de maneira aditiva no erro total, obtemos o modelo:

$$
y_{i j k}=\mu+e_{i}+e_{i j}+e_{i j k}, \quad i=1, \ldots, I ; j=1, \ldots J ; k=1, \ldots, K
$$

onde $y_{i j k}$ é um resultado obtido; $\mu$ é um parâmetro de locação comum a todas as observações; $e_{i}$ é o erro aleatório associado a lotes; $e_{i j}$ é o erro aleatório associado a amostras e $e_{i j k}$ é erro aleatório associado a análises químicas.

Em geral, considera-se que as variáveis aleatórias $e_{i j k}, e_{i j}$ e $e_{i}$ têm distribuições normais independentes e que

$$
\begin{aligned}
& E\left(e_{i j k}\right)=E\left(e_{i j}\right)=E\left(e_{i}\right)=0, \\
& \operatorname{Var}\left(e_{i j k}\right)=\sigma_{1}^{2}, \quad \operatorname{Var}\left(e_{i j}\right)=\sigma_{2}^{2} \text { e } \operatorname{Var}\left(e_{i}\right)=\sigma_{3}^{2} .
\end{aligned}
$$

Portanto, a variância de um resultado qualquer obtido é dada por

$$
\operatorname{Var}\left(y_{i j k}\right)=\sigma_{1}^{2}+\sigma_{2}^{2}+\sigma_{3}^{2}
$$

Usualmente as variáveis aleatórias $e_{i j}$ e $e_{i}$ são chamadas de efeitos aleatórios e o modelo em (1.3) é conhecido como modelo de efeitos aleatórios ( ver por exemplo, Box e Tiao, 1973). 


\subsubsection{Análise de Variância para o Modelo de Efeitos Aleatórios com 3 Componentes de Variância}

Estimadores clássicos usuais dos componentes de variância são facilmente obtidos quando agrupamos os dados em forma de quadro de análise de variância.

Daqui por diante usaremos a notação pela qual um ponto colocado no lugar do índice subscrito indica a média sobre o tal índice omitido, ou seja,

$$
y_{i j .}=\frac{1}{K} \sum_{k=1}^{K} y_{i j k}, \quad y_{i . .}=\frac{1}{J} \sum_{j=1}^{J} y_{i j .} \text { e } y_{\ldots . .}=\frac{1}{I} \sum_{i=1}^{I} y_{i . .} .
$$

De acordo com esta notação, podemos escrever a soma de quadrados total corrigida para o modelo (1.3) da forma:

$$
\sum_{i=1}^{I} \sum_{j=1}^{J} \sum_{k=1}^{K}\left(y_{i j k}-y_{\ldots . .}\right)^{2}=\sum_{i=1}^{I} \sum_{j=1}^{J} \sum_{k=1}^{K}\left[\left(y_{i . .}-y_{\ldots .}\right)+\left(y_{i j .}-y_{i . .}\right)+\left(y_{i j k}-y_{i j .}\right)\right]^{2} .
$$

Expandindo o lado direito da equação (1.4) e fazendo algumas simplificações obtemos a expressão

$$
\sum_{i=1}^{I} \sum_{j=1}^{J} \sum_{k=1}^{K}\left(y_{i j k}-y_{. . .}\right)^{2}=J K \sum_{i=1}^{I}\left(y_{i . .}-y_{. . .}\right)^{2}+K \sum_{i=1}^{I} \sum_{j=1}^{J}\left(y_{i j .}-y_{i . .}\right)^{2}+\sum_{i=1}^{I} \sum_{j=1}^{J} \sum_{k=1}^{K}\left(y_{i j k}-y_{i j .}\right)^{2}
$$

Simbolicamente, a equação (1.5) pode ser escrita na forma:

$$
S Q T=S_{3}+S_{2}+S_{1}
$$


ou seja, a soma de quadrados total ( SQT) pode ser decomposta em somas devido a três fontes de variação distintas: $S_{3}$, que é a soma de quadrados devido ao primeiro nível de amostragem (lotes, no exemplo); $S_{2}$, soma de quadrados devido ao segundo nível de amostragem (amostras) e $S_{1}$, soma de quadrados devido ao terceiro nível de amostragem (análises químicas).

O quadro de análise de variância apropriado para o modelo (1.3) é dado na tabela (1.2) (ver por exemplo, Box e Tiao, 1973; Box, Hunter e Hunter, 1978), onde $v_{1}=I J(K-1), v_{2}=I(J-1)$ e $v_{3}=I-1$, são os graus de liberdade associados a $S_{1}, S_{2}$ e $S_{3}$ respectivamente, e $m_{1}, m_{2}$ e $m_{3}$ são os quadrados médios associados às somas de quadrado.

Para obter os valores esperados das somas de quadrado recorremos a um teorema bastante conhecido na inferência clássica, dado a seguir.

Teorema 1.2. Sejam $x_{1}, x_{2}, \ldots, x_{n}, \mathrm{n}$ observações independentes de uma distribuição normal $N\left(0 ; \sigma^{2}\right)$ e seja $\bar{x}$ a média amostral e $\left(x_{i}-\bar{x}\right) \quad(i=1, \ldots, n)$ os resíduos. Então:

1) $\bar{x}$ tem distribuição normal $N\left(0 ; \sigma^{2} / n\right)$ e é independente de $\left(x_{i}-\bar{x}\right)$.

2) $\sum_{i=1}^{n}\left(x_{i}-\bar{x}\right)^{2}$ tem distribuição $\sigma^{2} \chi_{n-1}^{2}$, onde $\chi_{\mathrm{n}-1}^{2}$ é a distribuição Qui-Quadrado com $n-1$ graus de liberdade.

3) $\sum_{i=1}^{n}\left(x_{i}-\bar{x}\right)^{2}$ é uma estatística suficiente para $\sigma^{2}$.

Uma vez que estamos supondo normalidade e independência para as variáveis $e_{i}$, $e_{i j}$ e $e_{i j k}$, ou seja, $e_{i j k} \sim N\left(0, \sigma_{1}^{2}\right), e_{i j} \sim N\left(0 ; \sigma_{2}^{2}\right)$ e $e_{i} \sim N\left(0 ; \sigma_{3}^{2}\right)$, independentes, de acordo com o teorema (1.2) temos os seguintes resultados:

a) $y_{i . .} \sim N\left(\mu ; \sigma_{123}^{2} / J K\right)$,

b) $J K \sum_{i=1}^{I}\left(y_{i . .}-y_{\ldots .}\right)^{2}=v_{3} m_{3} \sim \sigma_{123}^{2} \chi_{v_{3}}^{2}$, 
c) $K \sum_{i=1}^{I} \sum_{j=1}^{J}\left(y_{i j .}-y_{i . .}\right)^{2}=v_{2} m_{2} \sim \sigma_{12}^{2} \chi_{v_{2}}^{2}$,

d) $\sum_{i=1}^{I} \sum_{j=1}^{J} \sum_{k=1}^{K}\left(y_{i j k}-y_{i j .}\right)^{2}=v_{1} m_{1} \sim \sigma_{1}^{2} \chi_{v_{1}}^{2}$,

e) As quantidades $\sum_{i=1}^{I} \sum_{j=1}^{J} \sum_{k=1}^{K}\left(y_{i j k}-y_{i j .}\right)^{2}$, e $K \sum_{i=1}^{I} \sum_{j=1}^{J}\left(y_{i j .}-y_{i . .}\right)^{2}$ são estatísticas suficientes para $\sigma_{1}^{2}$ e $\sigma_{12}^{2}$ respectivamente,

onde $\chi_{p}^{2}$ é a distribuição Qui-Quadrado com $p$ graus de liberdade e $\sigma_{12}^{2}=\sigma_{1}^{2}+K \sigma_{2}^{2}$ e $\sigma_{123}^{2}=\sigma_{1}^{2}+K \sigma_{2}^{2}+J K \sigma_{3}^{2}$.

A partir desses resultados podemos obter facilmente os valores esperados dos quadrados médios $m_{1}, m_{2}$ e $m_{3}$, e os estimadores clássicos usuais não viciados dos componentes de variância $\left(\sigma_{1}^{2}, \sigma_{2}^{2}, \sigma_{3}^{2}\right)$ são facilmente obtidos resolvendo-se as equações dos valores esperados desses quadrados médios (observar tabela 1.2), as quais fornecem os valores:

$$
\begin{gathered}
\hat{\sigma}_{1}^{2}=m_{1}, \\
\hat{\sigma}_{2}^{2}=\frac{m_{2}-m_{1}}{K}, \\
\hat{\sigma}_{3}^{2}=\frac{m_{3}-m_{2}}{J K} .
\end{gathered}
$$


Tabela (1.2) - Análise de variância para o modelo de efeitos aleatórios com 3 componentes de variância.

\begin{tabular}{|c|cccc||}
\hline Fonte de.Var. & S.Q. & G.L. & Q.M. & Valor Esp. do Q.M. \\
\hline Lotes & $S_{3}=J K \sum_{i=1}^{I}\left(y_{i . .}-y_{\ldots . .}\right)^{2}$ & $v_{3}$ & $m_{3}=S_{3} / v_{3}$ & $\sigma_{123}^{2}=\sigma_{1}^{2}+K \sigma_{2}^{2}+J K \sigma_{3}^{2}$ \\
Amostras & $S_{2}=K \sum_{i=1}^{I} \sum_{j=1}^{J}\left(y_{i j .}-y_{i . .}\right)^{2}$ & $v_{2}$ & $m_{2}=S_{2} / v_{2}$ & $\sigma_{12}^{2}=\sigma_{1}^{2}+K \sigma_{2}^{2}$ \\
Análises & $S_{1}=\sum_{i=1}^{I} \sum_{j=1}^{J} \sum_{k=1}^{K}\left(y_{i j k}-y_{i j .}\right)^{2}$ & $v_{1}$ & $m_{1}=S_{1} / v_{1}$ & $\sigma_{1}^{2}$ \\
Total & $\sum_{i=1}^{I} \sum_{j=1}^{J} \sum_{k=1}^{K}\left(y_{i j k}-y_{. . .}\right)^{2}$ & & & \\
\hline
\end{tabular}

\subsection{Modelo de Efeitos Aleatórios com 2 Componentes de Variância}

Uma situação especial que analisamos a seguir é dada quando apenas duas fontes de variação estão envolvidas num estudo. Para esse caso devemos considerar um planejamento hierárquico em dois níveis de amostragem.

Admitindo que para o primeiro nível do experimento sejam selecionadas aleatoriamente $J$ unidades amostrais e, num segundo nível, $K$ sub-unidades amostrais (ou réplicas) sejam retiradas de cada amostra, então, na suposição que amostras e subamostras variam independentemente e aditivamente contribuam com erros $e_{j}$ e $e_{j k}$ respectivamente, teremos o modelo:

$$
y_{j k}=\mu+e_{j}+e_{j k}, \quad j=1, \ldots J ; k=1, \ldots, K,
$$


onde $y_{j k}$ são as observações; $\mu$ é o parâmetro de locação comum a todas as observações; $e_{j}$ é o erro, ou efeito aleatório relacionado ao primeiro nível de amostragem e $e_{j k}$ é o erro aleatório relacionado ao segundo nível de amostragem. Observe que o modelo (1.8) é um caso especial do modelo (1.3) para $I=1$.

Analogamente ao modelo com 3 componentes de variância, as suposições usuais para o modelo (1.8) são que as variáveis aleatórias $e_{j k}$ e $e_{j}$ têm distribuições normais independentes com

$$
\begin{gathered}
E\left(e_{j k}\right)=E\left(e_{j}\right)=0, \\
\operatorname{Var}\left(e_{j k}\right)=\sigma_{1}^{2} \text { e } \operatorname{Var}\left(e_{j}\right)=\sigma_{2}^{2} .
\end{gathered}
$$

Conseqüentemente, a variância de uma observação qualquer é dada por

$$
\operatorname{Var}\left(y_{j k}\right)=\sigma_{1}^{2}+\sigma_{2}^{2}
$$

\subsubsection{Análise de Variância para o Modelo de Efeitos Aleatórios com 2 Componentes de Variância}

Usando o procedimento considerado anteriormente, a soma de quadrados total corrigida para o modelo (1.8) pode ser escrita na forma:

$$
\sum_{j=1}^{J} \sum_{k=1}^{K}\left(y_{j k}-y_{. .}\right)^{2}=K \sum_{j=1}^{J}\left(y_{j .}-y_{. .}\right)^{2}+\sum_{j=1}^{J} \sum_{k=1}^{K}\left(y_{j k}-y_{j .}\right)^{2} .
$$

Simbolicamente, a equação (1.9) pode ser expressa na forma: 


$$
S Q T=S_{2}+S_{1}
$$

onde $S_{2}$ e $S_{1}$ são as somas de quadrados devido ao primeiro e segundo nível de amostragem respectivamente.

Um quadro de análise de variância apropriado para o modelo (1.8) é apresentado na tabela (1.3) (ver por exemplo, Box e Tiao, 1973), onde $v_{1}=J(K-1)$ e $v_{2}=J-1$ são os graus de liberdade associados a $S_{1}$ e $S_{2}$ respectivamente, e $m_{1}$ e $m_{2}$ são os quadrados médios correspondentes às somas de quadrados.

Uma vez que estamos supondo normalidade e independência das variáveis $\left(e_{j}, e_{j k}\right)$ e, de acordo com os resultados do teorema (1.2), temos que:

a) As quantidades $y_{j}$. são independentes, tendo cada uma distribuição normal $N\left(\mu, \sigma_{12}^{2} / K\right)$, onde $\sigma_{12}^{2}=\sigma_{1}^{2}+K \sigma_{2}^{2}($ ver tabela 1.3$)$,

b) A quantidade $\sum_{k=1}^{K}\left(y_{j k}-y_{j}\right)^{2}$ têm distribuição $\sigma_{1}^{2} \chi_{(K-1)}^{2}$; conseqüentemente a soma $\sum_{j=1}^{J} \sum_{k=1}^{K}\left(y_{j k}-y_{j}\right)^{2}=S_{1}=m_{1} v_{1}$ tem distribuição $\sigma_{1}^{2} \chi_{\left(v_{1}\right)}^{2}$,

c) $v_{1} m_{1}$ é uma estatística suficiente para $\sigma_{1}^{2}$.

Tabela (1.3). Análise de Variância para o modelo de efeitos aleatórios com 2 componentes de variância.

\begin{tabular}{||l|cccl||}
\hline Fonte de Var. & S.Q. & G.L. & Q.M. & Valor Esp. Do Q.M. \\
\hline Amostras & $S_{2}=K \sum_{J=1}^{J}\left(y_{j .}-y_{.}\right)^{2}$ & $v_{2}$ & $m_{2}=S_{2} / v_{2}$ & $\sigma_{12}^{2}=\sigma_{1}^{2}+K \sigma_{2}^{2}$ \\
Sub-amostras & $S_{1}=\sum_{j=1}^{J} \sum_{k=1}^{K}\left(y_{j k}-y_{j}\right)^{2}$ & $v_{1}$ & $m_{1}=S_{1} / v_{1}$ & $\sigma_{1}^{2}$ \\
Total & $\sum_{j=1}^{J} \sum_{k=1}^{K}\left(y_{j k}-y_{. .}\right)^{2}$ & & & \\
\hline
\end{tabular}


Procedendo como no caso anterior (modelo com 3 componentes de variância ) obtemos estimadores clássicos usuais não viciados dos componentes de variância $\left(\sigma_{1}^{2}, \sigma_{2}^{2}\right)$, a partir da tabela de análise de variância, dados por

$$
\begin{gathered}
\hat{\sigma}_{1}^{2}=m_{1}, \\
\hat{\sigma}_{2}^{2}=\frac{m_{2}-m_{1}}{K} .
\end{gathered}
$$

\subsubsection{Função de Verossimilhança}

Para obtermos a função de verossimilhança é mais conveniente trabalharmos com os grupos de médias $y_{j}$ e os resíduos $\left(y_{j k}-y_{j .}\right)$ do que com as observações $y_{j k}$ apenas. Em termos do modelo (1.8) temos

$$
\begin{aligned}
& y_{j .}=\mu+e_{j}+e_{j .} \mathrm{e} \\
& y_{j k}-y_{j .}=e_{j k}-e_{j .},
\end{aligned}
$$

onde $e_{j .}=\frac{1}{K} \sum_{k} e_{j k}$

A função de verossimilhança para $\left(\mu, \sigma_{1}^{2}, \sigma_{12}^{2}\right)$, de acordo com os resultados decorrentes do teorema (1.2) é dada por

$$
L\left(\mu, \sigma_{1}^{2}, \sigma_{12}^{2} / y\right) \propto\left(\sigma_{1}^{2}\right)^{-\frac{\left(\eta_{1}\right)}{2}}\left(\sigma_{12}^{2}\right)^{-\frac{\left(v_{2}+1\right)}{2}} \exp \left\{-\frac{1}{2}\left[\frac{K \sum_{j=1}^{J}\left(y_{j .}-\mu\right)^{2}}{\sigma_{12}^{2}}+\frac{v_{1} m_{1}}{\sigma_{1}^{2}}\right]\right\} .
$$


Alternativamente, podemos expressar (1.12) em termos dos parâmetros $\left(\mu, \sigma_{1}^{2}, \sigma_{2}^{2}\right)$, observando que

$$
K \sum_{j=1}^{J}\left(y_{j .}-\mu\right)^{2}=v_{2} m_{2}+J K\left(y_{. .}-\mu\right)^{2}
$$

e portanto,

$$
L\left(\mu, \sigma_{1}^{2}, \sigma_{2}^{2} / y\right) \propto\left(\sigma_{1}^{2}\right)^{-\frac{\left(\nu_{1}\right)}{2}}\left(\sigma_{1}^{2}+K \sigma_{2}^{2}\right)^{-\frac{\left(\nu_{2}+1\right)}{2}} \exp \left\{-\frac{1}{2}\left[\frac{J K(y-\mu)^{2}}{\sigma_{1}^{2}+K \sigma_{2}^{2}}+\frac{v_{2} m_{2}}{\sigma_{1}^{2}+K \sigma_{2}^{2}}+\frac{v_{1} m_{1}}{\sigma_{1}^{2}}\right]\right\} .
$$

\subsubsection{Estimadores de Máxima Verossimilhança.}

A partir do momento em que coletamos os dados, toda a informação sobre os parâmetros está contida na função de verossimilhança (ou na função logverossimilhança).

O logaritmo da função de verossimilhança para $\mu, \sigma_{1}^{2}, \sigma_{2}^{2}$ é dado por

$$
l\left(\mu, \sigma_{1}^{2}, \sigma_{2}^{2} / y\right) \propto-\frac{v_{1}}{2} \ln \sigma_{1}^{2}-\frac{\left(v_{2}+1\right)}{2} \ln \left(\sigma_{1}^{2}+K \sigma_{2}^{2}\right)-\frac{1}{2}\left[\frac{J K(y . \mu-\mu)^{2}}{\sigma_{1}^{2}+K \sigma_{2}^{2}}+\frac{v_{2} m_{2}}{\sigma_{1}^{2}+K \sigma_{2}^{2}}+\frac{v_{1} m_{1}}{\sigma_{1}^{2}}\right] .
$$

As primeiras derivadas de $l\left(\mu, \sigma_{1}^{2}, \sigma_{2}^{2} / y\right)$ com respeito a $\mu, \sigma_{1}^{2}, \sigma_{2}^{2}$ são dadas por 


$$
\begin{aligned}
& \frac{\partial l\left(\mu, \sigma_{1}^{2}, \sigma_{2}^{2} / y\right)}{\partial \mu}=\frac{J K(y-\mu)}{\sigma_{1}^{2}+K \sigma_{2}^{2}} \\
& \frac{\partial l\left(\mu, \sigma_{1}^{2}, \sigma_{2}^{2} / y\right)}{\partial \sigma_{1}^{2}}=-\frac{v_{1}}{2 \sigma_{1}^{2}}-\frac{\left(v_{2}+1\right)}{2\left(\sigma_{1}^{2}+K \sigma_{2}^{2}\right)}+\frac{J K(y-\mu)^{2}}{2\left(\sigma_{1}^{2}+K \sigma_{2}^{2}\right)^{2}}+\frac{v_{2} m_{2}}{2\left(\sigma_{1}^{2}+K \sigma_{2}^{2}\right)^{2}}+\frac{v_{1} m_{1}}{2\left(\sigma_{1}^{2}\right)^{2}} \mathrm{e} \\
& \frac{\partial l\left(\mu, \sigma_{1}^{2}, \sigma_{2}^{2} / y\right)}{\partial \sigma_{2}^{2}}=-\frac{\left(v_{2}+1\right) K}{2\left(\sigma_{1}^{2}+K \sigma_{2}^{2}\right)}+\frac{J K^{2}(y-\mu)^{2}}{2\left(\sigma_{1}^{2}+K \sigma_{2}^{2}\right)^{2}}+\frac{v_{2} m_{2} K}{2\left(\sigma_{1}^{2}+K \sigma_{2}^{2}\right)^{2}} .
\end{aligned}
$$

Igualando a zero as primeiras derivadas (1.16), (1.17) e (1.18) e resolvendo o sistema de equações em $\mu, \sigma_{1}^{2}, \sigma_{2}^{2}$, obtemos os Estimadores de Máxima Verossimilhança (EMV) de $\mu, \sigma_{1}^{2}, \sigma_{2}^{2}$ os quais são:

$$
\begin{gathered}
\hat{\mu}=y_{. .,} \\
\hat{\sigma}_{1}^{2}=m_{1}, \\
\hat{\sigma}_{2}^{2}=\left(\frac{v_{2} m_{2}}{v_{2}+1}-m_{1}\right) / K .
\end{gathered}
$$

Observando (1.19) vemos que o estimador de máxima verossimilhança de $\sigma_{2}^{2}$ é um estimador viciado e que em (1.10) temos um estimador corrigido.

\subsection{Inferências sobre os Parâmetros $\sigma_{1}^{2}$ e $\sigma_{2}^{2}$}

As distribuições amostrais das somas de quadrado $S_{1}$ e $S_{2}$ do modelo de efeitos aleatórios (1.8) podem ser utilizadas para obter intervalos de confiança para $\sigma_{1}^{2}$ e para a razão $\sigma_{2}^{2} / \sigma_{1}^{2}$. 
De acordo com os resultados do teorema (1.2) temos que a densidade para

$$
\frac{\sum_{j} \sum_{i}\left(y_{j k}-y_{j .}\right)^{2}}{\sigma_{1}^{2}}=\frac{S_{1}}{\sigma_{1}^{2}}=\frac{m_{1} v_{1}}{\sigma_{1}^{2}}
$$

é a densidade de uma distribuição Qui-Quadrado com $v_{1}$ graus de liberdade. Daí, um intervalo de confiança $100(1-\alpha) \%$ para $\sigma_{1}^{2}$ é dado por

$$
\left(\frac{m_{1} v_{1}}{\chi_{v_{1}(1-\alpha / 2)}^{2}} ; \frac{m_{1} v_{1}}{\chi_{v_{1}(\alpha / 2)}^{2}}\right),
$$

onde $\chi_{v_{1}(\alpha / 2)}^{2}$ é o quantil da distribuição Qui-Quadrado dado por

$$
P\left(\chi_{v_{1}}^{2} \leq \chi_{v_{1}(\alpha / 2)}^{2}\right)=\frac{\alpha}{2}
$$

Também, de acordo com o teorema (1.2) temos que a densidade para

$$
\frac{K \sum_{j}\left(y_{j .}-y_{. .}\right)^{2}}{\sigma_{12}^{2}}=\frac{S_{2}}{\sigma_{12}^{2}}=\frac{m_{2} v_{2}}{\sigma_{12}^{2}}
$$

é a densidade de uma distribuição Qui-Quadrado com $v_{2}$ graus de liberdade. Conseqüentemente, a densidade para a razão

$$
\frac{m_{1} \sigma_{12}^{2}}{m_{2} \sigma_{1}^{2}}=\frac{m_{1}}{m_{2}}\left(1+K \frac{\sigma_{2}^{2}}{\sigma_{1}^{2}}\right),
$$


é a densidade de uma distribuição $F$ com $v_{1}$ e $v_{2}$ graus de liberdade (ver por exemplo, Mood, Graybill e Boes, 1974).

Portanto, um intervalo de confiança $100(1-\alpha) \%$ para a razão $\sigma_{2}^{2} / \sigma_{1}^{2}$ é dado por

$$
\left[\left(\frac{m_{2}}{m_{1}} F_{v_{1}, v_{2}(\alpha / 2)}-1\right) / K ;\left(\frac{m_{2}}{m_{1}} F_{v_{1}, v_{2}(1-\alpha / 2)}-1\right) / K\right]
$$

onde $F_{v_{1}, v_{2}(\alpha / 2)}$ é o quantil da distribuição $F$ dado por

$$
P\left(F_{v_{1}, v_{2}} \leq F_{v_{1}, v_{2}(\alpha / 2)}\right)=\frac{\alpha}{2} .
$$

A distribuição normal assintótica dos estimadores de máxima verossimilhança (ver por exemplo, Mood, Graybill e Boes, 1974) também pode ser utilizada para obtermos inferências sobre os parâmetros do modelo, ou mesmo para funções dos mesmos. Para amostras grandes temos que

$$
\left(\hat{\mu}, \hat{\sigma}_{1}^{2}, \hat{\sigma}_{2}^{2}\right) \stackrel{a}{\sim} N\left[\left(\mu, \sigma_{1}^{2}, \sigma_{2}^{2}\right) ; I^{-1}\left(\hat{\mu}, \hat{\sigma}_{1}^{2}, \hat{\sigma}_{2}^{2}\right)\right]
$$

onde $I\left(\mu, \sigma_{1}^{2}, \sigma_{2}^{2}\right)$ é a matriz de informação de Fisher, a qual é constituída pelas esperanças das segundas derivadas parciais de $l\left(\mu, \sigma_{1}^{2}, \sigma_{2}^{2} / y\right)$, a menos do sinal (ver por exemplo, Box e Tiao, 1973).

As segundas derivadas parciais da função log-verossimilhança (1.15) com respeito aos parâmetros $\mu, \sigma_{1}^{2}, \sigma_{2}^{2}$ são dados por 


$$
\frac{\partial^{2} l\left(\mu, \sigma_{1}^{2}, \sigma_{2}^{2} / y\right)}{\partial \mu^{2}}=-\frac{J K}{\sigma_{1}^{2}+K \sigma_{2}^{2}},
$$

$$
\frac{\partial^{2} l\left(\mu, \sigma_{1}^{2}, \sigma_{2}^{2} / y\right)}{\partial\left(\sigma_{1}^{2}\right)^{2}}=\frac{v_{1}}{2\left(\sigma_{1}^{2}\right)^{2}}+\frac{\left(v_{2}+1\right)}{2\left(\sigma_{1}^{2}+K \sigma_{2}^{2}\right)^{2}}-\frac{J K(y-\mu)^{2}}{\left(\sigma_{1}^{2}+K \sigma_{2}^{2}\right)^{3}}-\frac{v_{2} m_{2}}{\left(\sigma_{1}^{2}+K \sigma_{2}^{2}\right)^{3}}-\frac{v_{1} m_{1}}{\left(\sigma_{1}^{2}\right)^{3}},
$$

$$
\frac{\partial^{2} l\left(\mu, \sigma_{1}^{2}, \sigma_{2}^{2} / y\right)}{\partial\left(\sigma_{2}^{2}\right)^{2}}=\frac{K^{2}\left(v_{2}+1\right)}{2\left(\sigma_{1}^{2}+K \sigma_{2}^{2}\right)^{2}}-\frac{J K^{3}(y-\mu)^{2}}{\left(\sigma_{1}^{2}+K \sigma_{2}^{2}\right)^{3}}-\frac{K^{2} v_{2} m_{2}}{\left(\sigma_{1}^{2}+K \sigma_{2}^{2}\right)^{3}},
$$

$\frac{\partial^{2} l\left(\mu, \sigma_{1}^{2}, \sigma_{2}^{2} / y\right)}{\partial \mu \partial \sigma_{1}^{2}}=-\frac{J K\left(y_{. .}-\mu\right)}{\left(\sigma_{1}^{2}+K \sigma_{2}^{2}\right)^{2}}$,

$$
\frac{\partial^{2} l\left(\mu, \sigma_{1}^{2}, \sigma_{2}^{2} / y\right)}{\partial \mu \partial \sigma_{2}^{2}}=-\frac{J K^{2}\left(y_{-.}-\mu\right)}{\left(\sigma_{1}^{2}+K \sigma_{2}^{2}\right)^{2}}
$$

$$
\frac{\partial^{2} l\left(\mu, \sigma_{1}^{2}, \sigma_{2}^{2} / y\right)}{\partial \sigma_{1}^{2} \partial \sigma_{2}^{2}}=\frac{K\left(v_{2}+1\right)}{2\left(\sigma_{1}^{2}+K \sigma_{1}^{2}\right)^{2}}-\frac{J K^{2}(y-\mu)^{2}}{\left(\sigma_{1}^{2}+K \sigma_{2}^{2}\right)^{3}}-\frac{K v_{2} m_{2}}{\left(\sigma_{1}^{2}+K \sigma_{2}^{2}\right)^{3}} .
$$

Para o cálculo da esperança das expressões de (1.22) a (1.27) devemos lembrar os resultados decorrentes das suposições sobre o modelo (1.8), de onde temos que:

$$
\begin{aligned}
& E\left[-\frac{\partial^{2} l\left(\mu, \sigma_{1}^{2}, \sigma_{2}^{2} / y\right)}{\partial \mu^{2}}\right]=\frac{J K}{\sigma_{1}^{2}+K \sigma_{2}^{2}}, \\
& E\left[-\frac{\partial^{2} l\left(\mu, \sigma_{1}^{2}, \sigma_{2}^{2} / y\right)}{\partial\left(\sigma_{1}^{2}\right)^{2}}\right]=\frac{v_{1}}{2\left(\sigma_{1}^{2}\right)^{2}}+\frac{\left(v_{2}+1\right)}{2\left(\sigma_{1}^{2}+K \sigma_{2}^{2}\right)^{2}},
\end{aligned}
$$




$$
\begin{aligned}
& E\left[-\frac{\partial^{2} l\left(\mu, \sigma_{1}^{2}, \sigma_{2}^{2} / y\right)}{\partial\left(\sigma_{2}^{2}\right)^{2}}\right]=\frac{K^{2}\left(v_{2}+1\right)}{2\left(\sigma_{1}^{2}+K \sigma_{2}^{2}\right)^{2}}, \\
& E\left[-\frac{\partial^{2} l\left(\mu, \sigma_{1}^{2}, \sigma_{2}^{2} / y\right)}{\partial \sigma_{1}^{2} \partial \sigma_{2}^{2}}\right]=\frac{K\left(v_{2}+1\right)}{2\left(\sigma_{1}^{2}+K \sigma_{2}^{2}\right)^{2}}, \\
& E\left[-\frac{\partial^{2} l\left(\mu, \sigma_{1}^{2}, \sigma_{2}^{2} / y\right)}{\partial \mu \partial \sigma_{1}^{2}}\right]=E\left[-\frac{\partial^{2} l\left(\mu, \sigma_{1}^{2}, \sigma_{2}^{2}\right)}{\partial \mu \partial \sigma_{2}^{2}}\right]=0 .
\end{aligned}
$$

Portanto, a matriz de Informação de Fisher para $\mu, \sigma_{1}^{2}, \sigma_{2}^{2}$ é dada por

$$
I\left(\mu, \sigma_{1}^{2}, \sigma_{2}^{2}\right)=\left[\begin{array}{ccc}
\frac{J K}{\sigma_{1}^{2}+K \sigma_{2}^{2}} & 0 & 0 \\
& \frac{v_{1}}{2\left(\sigma_{1}^{2}\right)^{2}}+\frac{\left(v_{2}+1\right)}{2\left(\sigma_{1}^{2}+K \sigma_{2}^{2}\right)^{2}} & \frac{K\left(v_{2}+1\right)}{2\left(\sigma_{1}^{2}+K \sigma_{2}^{2}\right)^{2}} \\
\text { simétrica } & \frac{K^{2}\left(v_{2}+1\right)}{2\left(\sigma_{1}^{2}+K \sigma_{2}^{2}\right)^{2}}
\end{array}\right] .
$$

Observando a matriz de Informação de Fisher (1.28) vemos que seus elementos dependem dos parâmetros $\sigma_{1}^{2}$ e $\sigma_{2}^{2}$, implicando numa aproximação assintótica Normal, em geral, imprecisa para os estimadores de máxima verossimilhança de $\sigma_{1}^{2}$ e $\sigma_{2}^{2}$, especialmente para amostras pequenas e moderadas (ver por exemplo, Sprott, 1973, 1980):

Podemos construir intervalos de confiança para os parâmetros do modelo (1.8), considerando um coeficiente de confiança $100(1-\alpha) \%$, da seguinte forma:

$$
P\left[\hat{\sigma}_{i}^{2}-z_{\alpha / 2} \sqrt{I_{i i}^{-1}\left(\mu, \sigma_{1}^{2}, \sigma_{2}^{2}\right)} \leq \sigma_{i}^{2} \leq \hat{\sigma}_{i}^{2}+z_{\alpha / 2} \sqrt{I_{i i}^{-1}\left(\mu, \sigma_{1}^{2}, \sigma_{2}^{2}\right)}\right]=1-\alpha,
$$


onde $I_{i i}^{-1}\left(\mu, \sigma_{1}^{2}, \sigma_{2}^{2}\right)$ representa o i-ésimo elemento diagonal da matriz de variầncia e covariância assintótica de $\hat{\mu}, \hat{\sigma}_{1}^{2}$ e $\hat{\sigma}_{2}^{2}$ e $z_{\alpha / 2}$ é um percentil da distribuição normal padrão.

Podemos observar que as inferências sobre os parâmetros do modelo são todas baseadas na suposição de normalidade. Quando essas suposições não são verificadas as inferências sobre os parâmetros podem estar comprometidas. Além disso, temos que na prática $e_{j}$ representa uma flutuação vinda de uma fonte suspeita em particular, enquanto que $e_{j k}$ representa uma soma de distúrbios residuais, as quais podem estar vindo de um número de fontes independentes. Portanto, podemos esperar que existe uma tendência da distribuição de $e_{j k}$ se aproximar da distribuição normal. Por outro lado, uma fonte particular de variação pode levar a distribuição de $e_{j}$ muito diferente da normal.

Assumindo o modelo com dois componentes de variância (1.8), apresentamos a seguir um modelo alternativo ao usualmente utilizado em problemas de análise de variância.

\subsection{Modelo de Efeitos Aleatórios Assumindo Não- Normalidade}

Uma situação que pode ser mais realística que a assumida até o momento é quando consideramos que os efeitos aleatórios $e_{j}$, em (1.8), não são normalmente distribuídos. Mais especificamente, de acordo com o modelo (1.8), vamos assumir que a variável aleatória $e_{j k}$ tem distribuição normal $N\left(0, \sigma_{1}^{2}\right)$ e que o efeito aleatório $e_{j}$ tem uma distribuição mistura de normais (ver Tiao e Ali, 1971), dada pela densidade

$$
\begin{aligned}
& p\left(e_{j} / \sigma_{2}^{2}, \theta, \delta, \lambda\right)=(1-\theta) f_{N}\left(e_{j} /-\delta \theta \sigma, \sigma^{2}\right)+ \\
& +\theta f_{N}\left(e_{j} /-\delta \theta \sigma+\delta \sigma, \lambda^{2} \sigma^{2}\right),
\end{aligned}
$$


onde $-\infty<e_{j}<\infty, \sigma>0,-\infty<\delta<\infty, \lambda \geq 1,0 \leq \theta \leq 1$, e $f_{N}(x / p, q)$ indica a densidade de uma distribuição normal com média $p$ e variância $q$.

O modelo em (1.30) é um caso especial de mistura de duas distribuições normais o qual já foi considerado por vários autores (ver por exemplo, Cohen, 1967; Hasselblad, 1966; Hill, 1965);

A distribuição em (1.30) pode ser interpretada como se $e_{j}$ viesse de uma entre duas populações, um modelo central $N\left(-\delta \theta \sigma, \sigma^{2}\right)$ e um modelo alternativo $N\left(-\delta \theta \sigma+\delta \sigma, \lambda^{2} \sigma^{2}\right)$, com probabilidades $(1-\theta)$ e $\theta$ respectivamente. O modelo alternativo representa uma possível mudança na média do processo por $\delta \sigma$ e uma alteração no desvio padrão por um fator $\lambda$.

Essas distribuições têm média zero e as expressões para a variância, medidas de assimetria e curtose $\gamma_{1}$ e $\gamma_{2}$ são dadas por

$$
\operatorname{var}\left(e_{j}\right)=\sigma_{2}^{2}=\sigma^{2}\left\{1+\theta\left(\lambda^{2}-1\right)+\delta^{2} \theta(1-\theta)\right\},
$$

$\gamma_{1}=\frac{\theta(1-\theta) \delta\left\{\delta^{2}(1-2 \theta)+3\left(\lambda^{2}-1\right)\right\}}{\left\{1+\theta\left(\lambda^{2}-1\right)+\theta(1-\theta) \delta^{2}\right\}^{3 / 2}} \mathrm{e}$

$$
\gamma_{2}=\frac{3\left\{1+\theta\left(\lambda^{4}-1\right)\right\}+6 \theta(1-\theta) \delta^{2}\left\{\theta+(1-\theta) \lambda^{2}\right\}+\delta^{4} \theta(1-\theta)\left\{\theta^{3}+(1-\theta)^{3}\right\}}{\left\{1+\theta\left(\lambda^{2}-1\right)+\theta(1-\theta) \delta^{2}\right\}^{2}} .
$$

Como um caso especial de distribuição (1.30), considere $\theta=0,05$, isto é, o processo está fora de controle em $5 \%$ das vezes. Também, assumindo $|\delta|=\lambda-1$, isto é $\delta=\phi(\lambda-1), \phi=-1,1$ temos o modelo (ver Tiao e Ali, 1971):

$$
\begin{aligned}
& p\left(e_{j} / \sigma_{2}^{2}, \lambda, \phi\right)=0,95 f_{N}\left(e_{j} /-0,05 \phi(\lambda-1) \sigma, \sigma^{2}\right)+ \\
& +0,05 f_{N}\left(e_{j} / 0,95 \phi(\lambda-1) \sigma, \lambda^{2} \sigma^{2}\right) .
\end{aligned}
$$


Observe que, se $\lambda=1 \mathrm{em}$ (1.32), temos a distribuição normal para $e_{j}$ (suposição usual). Se $\lambda>1$, a distribuição é simétrica mas leptocúrtica para $\phi=0$, desviada para direita para $\phi=1$, e desviada para a esquerda para $\phi=-1$.

Para o modelo geral (1.30), Tiao e Ali (1971) mostram que a distribuição é unimodal para todo $\theta$ em $(0,1)$ se e somente se

$$
\delta^{2}<\frac{27\left(1-\lambda^{-2}\right)^{2}}{\left(1-2 \lambda^{-2}\right)\left(2+\lambda^{-2}-\lambda^{-4}\right)+2\left(1-\lambda^{-2}+\lambda^{-4}\right)^{3 / 2}} .
$$

Problemas com mistura de distribuições encontram aplicações em diversas áreas como na engenharia, medicina, geologia, e outras. As análises de dados com essas distribuições podem não ser simples devido principalmente a dois problemas: primeiramente, porque não existem fórmulas explícitas para os estimadores de vários parâmetros do modelo sendo necessária a utilização de métodos numéricos na estimação dos parâmetros; em segundo lugar, as dificuldades teóricas que surgem em certos aspectos da análise estatística revelam que alguns problemas com mistura não apresentam um comportamento padrão (ver por exemplo, Titterington, Smith e Makov, 1985).

\subsection{Motivações e Objetivos}

Os métodos clássicos usuais para análise de componentes de variância, algumas vezes, podem encontrar sérias dificuldades. Várias alternativas foram sugeridas na literatura, mas nenhuma delas convence de maneira satisfatória os pesquisadores da área.

A seguir destacamos alguns problemas comuns que podem ocorrer. 


\section{i) Estimativa negativa dos componentes de variância.}

A estimativa clássica usual de $\sigma_{2}^{2}$ (observar 1.10) para o modelo com dois componentes de variância e as estimativas de $\sigma_{2}^{2}$ e $\sigma_{3}^{2}$ (observar 1.7) para o modelo com três componentes, são obtidas pela diferença de quadrados médios e portanto, podem apresentar valores negativos, o que representaria um grande problema pois, como sabemos, esses valores são- sempre não-negativos.

\section{ii) Dificuldades com intervalos de confiança.}

Considerando o modelo com dois componentes de variância (1.8), mesmo com a suposição que $e_{j}$ e $e_{j k}$ sejam normalmente distribuídos e independentes, a distribuição amostral de $\hat{\sigma}_{2}^{2}$ é bastante complicada e depende da razão de variâncias desconhecida $\sigma_{2}^{2} / \sigma_{1}^{2}$. O problema para obtenção de intervalos de confiança para $\sigma_{2}^{2}$ é complicado e nenhum procedimento generalizado é apresentado na literatura.

Também, na estimação da razão de variâncias $\sigma_{2}^{2} / \sigma_{1}^{2}$, os intervalos de confiança comumente utilizados (1.21), baseados na distribuição amostral da razão de quadrados médios $m_{2} / m_{1}$, podem incluir valores negativos. Uma possível alternativa seria restringir os valores de $\hat{\sigma}_{2}^{2}$ a valores não negativos (ver por exemplo, Herbach, $1959 \mathrm{e}$ Thompson, 1962; 1963) mas isso invalidaria a propriedade de estimadores não-viciados e complicaria ainda mais a distribuição de $\hat{\sigma}_{2}^{2}$.

\section{iii) Não verificação das suposições}

Outra dificuldade na estrutura tradicional é a sensibilidade das inferências quando as suposições básicas de normalidade e independência não são satisfeitas. Scheffé (1961) mostrou que a não-normalidade de $e_{j}$ e a falta de independência em $e_{j k}$ causam sérios efeitos nas distribuições que são utilizadas para fazer inferências sobre os parâmetros $\sigma_{1}^{2}$ e $\sigma_{2}^{2}$. 
Todas essas dificuldades agravam-se ainda mais com o aumento do número de parâmetros no modelo.

Diante desses problemas com o teoria clássica usual, o nosso objetivo é analisar o problema de componentes de variância sob o aspecto Bayesiano.

Consideraremos a princípio, um modelo com dois componentes de variância onde manteremos as suposições de normalidade e independência dos erros e utilizaremos densidades a priori não-informativas para os parâmetros do modelo. Duas densidades a priori serão consideradas: uma introduzida por Box e Tiao (1973) e outra obtida segundo a regra de Jeffreys. Também usaremos o método de aproximação de Laplace (ver por exemplo, Tierney e Kadane, 1986) e uma modificação do mesmo que será apresentada no capítulo 2, para aproximar as integrais quando não conseguimos soluções analíticas explícitas para densidades e momentos a posteriori de interesse.

Além disso, usaremos técnicas de diagnóstico de normalidade como, o gráfico T-Plot (ver por exemplo, Hills e Smith, 1993) e medidas de curvatura (ver por exemplo, Kass e Slate, 1992) para analisar a normalidade de densidades a posteriori, considerando a parametrização original $\left(\sigma_{1}^{2}, \sigma_{2}^{2}\right)$, e também considerando outra parametrização proposta por Achcar e Smith (1990).

Alguns resultados também serão obtidos considerando o modelo com três componentes de variância.

Assumindo o modelo com distribuição mistura de normais introduzido na seção (1.5), desenvolvemos uma análise Bayesiana aproximada utilizando o método de Laplace para aproximar integrais de interesse, assumindo diferentes densidades a priori para os parâmetros do modelo.

Para ilustrar as técnicas propostas, apresentaremos no capítulo 6, alguns exemplos de aplicações onde empregamos as metodologias obtidas. Finalmente, no capítulo 7 comentamos sobre algumas conclusões e perspectivas futuras desse trabalho. 


\section{Capítulo 2}

\section{Métodos para Aproximação de Integrais}

A utilização de métodos Bayesianos em geral envolve a resolução de integrais para calcular densidades a posteriori marginais, densidades preditivas ou momentos a posteriori de interesse. Em muitas situações não há possibilidade de se obter uma forma analítica explícita para essas integrais, principalmente quando não se trabalha com densidades a priori conjugadas, exigindo o uso de métodos de aproximações de integrais.

Nesse caso, o estatístico Bayesiano pode escolher uma entre as várias estratégias existentes como: o uso de métodos numéricos (ver por exemplo, Naylor e Smith, 1982); o uso de métodos de aproximação analítica para integrais (ver por exemplo, Tierney e Kadane, 1986); o uso do método de Monte Carlo ou Gibbs sampling (ver por exemplo, Kloek e Van Dijk, 1978; ou Gelfand e Smith, 1990).

Suponha que temos o interesse em calcular momentos a posteriori para funções $g(\psi)$ da forma: 


$$
E(g(\psi) / y)=\frac{\int g(\psi) \pi(\psi) L(\psi / y) d \psi}{\int \pi(\psi) L(\psi / y) d \psi},
$$

onde $g(\psi)$ é uma função selecionada de $\psi \in R^{m}, \pi(\psi)$ é uma densidade a priori e $L(\psi / y)$ é a função de verossimilhança para $\psi$ dado um vetor de dados $y$; e densidades a posteriori da forma:

$$
\pi\left(\psi_{1} / y\right)=\int \pi\left(\psi_{1}, \psi_{2} / y\right) d \psi_{2}
$$

onde $\pi\left(\psi_{1}, \psi_{2} / y\right)$ é uma densidade a posteriori conjunta para $\psi=\left(\psi_{1}, \psi_{2}\right), \psi_{1} \in R^{k} \mathrm{e}$ $\psi_{2} \in R^{m-k}$.

Quando não existe a possibilidade de determinar $E(g(\psi) / y)$ e $\pi\left(\psi_{1} / y\right)$ analiticamente, podemos utilizar um método de aproximação para resolver o problema.

\subsection{Método de Laplace para Aproximação de Integrais}

O método de aproximação para momentos a posteriori introduzido por Tierney e Kadane (1986) é baseado nas aproximações de Laplace para o numerador e denominador de (2.1). Essas aproximações assumem que a principal contribuição para as integrais relevantes está vindo de um máximo no interior do espaço paramétrico $\Psi$. O método de Laplace para aproximação de integrais é usado para resolver integrais da forma:

$$
I=\int f(\psi) \exp \{-n h(\psi)\} d \psi
$$


onde $-n h(\psi)$ é uma função com máximo em $\hat{\psi}$ e que satisfaz algumas condições usuais de regularidade.

Para aproximar integrais da forma (2.3), o método de Laplace assume uma expansão de $h$ e $f$ em série de Taylor em torno do máximo $\hat{\psi}$ de $-h$ (ver Tierney e Kadane, 1986; ou Kass, Tierney e Kadane, 1990).

Com $\psi$ unidimensional, a aproximação de Laplace para $I$ é dada por

$$
\hat{I} \cong\left(\frac{2 \pi}{n}\right)^{1 / 2} \sigma f(\hat{\psi}) \exp \{-n h(\hat{\psi})\}
$$

onde $\sigma=\left\{h^{\prime \prime}(\hat{\psi})\right\}^{-1 / 2}$.

No caso m-dimensional, ou seja, com $\psi \in R^{m}$ a aproximação de Laplace é dada por

$$
\hat{I} \cong(2 \pi)^{m / 2}\left\{\operatorname{det}\left(n \sum_{h}^{-1}(\hat{\psi})\right)\right\}^{-1 / 2} f(\hat{\psi}) \exp \{-n h(\hat{\psi})\},
$$

onde $\sum_{h}^{-1}(\hat{\psi})$ é a matriz Hessiana de $h$ calculada em $\hat{\psi}$, dada por

$$
\sum_{h}^{-1}(\hat{\psi})=\left[\begin{array}{cccc}
\frac{\partial^{2} h}{\partial \psi_{1}^{2}} & \frac{\partial^{2} h}{\partial \psi_{1} \partial \psi_{2}} & \cdots & \frac{\partial^{2} h}{\partial \psi_{1} \partial \psi_{m}} \\
& \frac{\partial^{2} h}{\partial \psi_{2}^{2}} & \cdots & \frac{\partial^{2} h}{\partial \psi_{2} \partial \psi_{m}} \\
& & \ddots & \vdots \\
\text { simétrica } & & & \frac{\partial^{2} h}{\partial \psi_{m}^{2}}
\end{array}\right] .
$$


Para aproximar o momento a posteriori (2.1), podemos considerar $\pi(\psi) L(\psi / y)=$ $\exp \{-n h(\psi)\}$ no numerador e denominador de (2.1), com $f$ iguais a $g$ e 1 , respectivamente. Assim, teremos uma aproximação modal da forma:

$$
E\{g(\psi) / y\}=g(\hat{\psi})\left\{1+\mathrm{O}\left(n^{-1}\right)\right\}
$$

onde $\hat{\psi}$ é a moda de $\pi(\psi / y)$.

Podemos considerar outras escolhas para $f$ em (2.3), nas integrais do numerador e denominador de (2.1) a fim de obtermos aproximações mais precisas para os momentos a posteriori em (2.1).

Uma escolha que proporciona maior precisão é dada por $f=1$ em ambas integrais em (2.1), ou seja,

$$
E(g(\psi) / y)=\frac{\int e^{-n h^{*}(\psi)} d \psi}{\int e^{-n h(\psi)} d \psi}
$$

onde $g(\psi)$ é uma função positiva, $-n h(\psi)=\ln \pi(\psi)+\ln L(\psi / y)$ e $-n h^{*}(\psi)=$ $\ln g(\psi)-n h(\psi)$. Quando $f=1$ em (2.3) o método de Laplace é conhecido como método de Laplace totalmente exponencial (ver por exemplo, Tierney e Kadane, 1986; ou Tierney, Kass e Kadane, 1989).

Usando o método de Laplace em (2.7) temos:

$$
\hat{E}(g(\psi) / y) \cong\left(\frac{\sigma^{*}}{\sigma}\right) \exp \left\{-\left[h^{*}\left(\hat{\psi}^{*}\right)-h(\hat{\psi})\right]\right\}
$$


onde $\hat{\psi}$ maximiza $-n h(\psi)$, e $\hat{\psi}^{*} \operatorname{maximiza}-n h^{*}(\psi), \sigma=\left\{\operatorname{det}\left(n \sum_{h}^{-1}(\hat{\psi})\right)\right\}^{-1 / 2}$ e $\sigma^{*}=\left\{\operatorname{det}\left(n \sum_{h^{*}}^{-1}\left(\hat{\psi}^{*}\right)\right)\right\}^{-1 / 2}$. Esta aproximação satisfaz:

$$
E(g(\psi) / y)=\hat{E}(g(\psi) / y)\left\{1+\mathrm{O}\left(n^{-2}\right)\right\}
$$

Analogamente, calculamos as aproximações de Laplace para a densidade a posteriori marginal (2.2).

Usualmente a aproximação em (2.8) apresenta grande precisão, principalmente para tamanhos amostrais $n$ grandes. Apesar disso, em muitos casos essa precisão pode ser melhorada considerando-se uma reparametrização adequada (ver por exemplo, Achcar e Smith, 1990; Kass e Slate, 1992). Para amostras pequenas a escolha de uma parametrização adequada é fundamental para se obter resultados mais precisos.

Dificuldades podem surgir quando o espaço paramétrico apresenta restrições levando a um 'corte' na densidade a posteriori $\pi(\psi / y)$. Nesses casos a moda a posteriori, sem considerar a restrição, ou seja o máximo irrestrito de $\pi(\psi / y)$, pode pertencer ou não ao espaço paramétrico $\Psi$, e isso pode levar a um grande erro ou mesmo à impossibilidade de se calcular as aproximações de Laplace para (2.1) e (2.2) (ver por exemplo Achcar e Pegorin, 1995). A seguir apresentamos uma generalização do método de aproximação de Laplace para o cálculo de momentos a posteriori quando ocorre esse tipo de problema.

\subsection{Aproximação de Laplace quando o Espaço Paramétrico Apresenta Restrições}

Considere o caso totalmente exponencial, $f=1$ em (2.3), e assumir $\psi$ unidimensional com um espaço paramétrico dado por $\Psi=\{\psi,-\infty<\psi<a\}$, onde $a$ é uma constante e considere uma aproximação para a integral 


$$
I_{1}=\int_{-\infty}^{a} e^{-n h(\psi)} d \psi
$$

onde o máximo $\hat{\psi}$ de $-h$ pode estar ou não no interior do espaço paramétrico.

Expandindo $h$ em série de Taylor em torno do máximo $\hat{\psi}$ de $-h$ temos

$$
\hat{I}_{1} \cong e^{-n h(\hat{\psi})} \int_{-\infty}^{a} e^{-\frac{n h^{\prime \prime}(\hat{\psi})(\psi-\hat{\psi})^{2}}{2}} d \psi
$$

A integral em (2.10) pode ser resolvida utilizando o seguinte resultado baseado na distribuição normal,

Resultado 2.1 : $\int_{-\infty}^{a} e^{-\frac{(x-\mu)^{2}}{2 \delta^{2}}} d x=\sqrt{2 \pi} \delta \Phi\left(\frac{a-\mu}{\delta}\right)$, onde $\Phi$ denota a função de distribuição de uma distribuição normal padrão $N(0,1)$.

Portanto, em (2.10) temos a aproximação

$$
\hat{I}_{2} \cong\left(\frac{2 \pi}{n}\right)^{1 / 2} \sigma e^{-n h(\hat{\psi})} \Phi\left(\frac{a-\hat{\psi}}{\sigma}\right)
$$

onde $\sigma=\left\{h^{\prime \prime}(\hat{\psi})\right\}^{-1 / 2}$.

Observe que se $(a-\hat{\psi}) / \sigma>3$, a aproximação de Laplace em (2.11) se reduz a (2.4), com $f=1$, pois nesse caso $\Phi\left(\frac{a-\hat{\psi}}{\sigma}\right) \cong 1$. Observe também que se o máximo 
está na fronteira do espaço paramétrico, ou seja, se $a=\hat{\psi}$, temos $\Phi\left(\frac{a-\hat{\psi}}{\sigma}\right)=\Phi(0)=1 / 2$, e nesse caso a aproximação de Laplace para $I_{2}$ é dada por

$$
\hat{I}_{2} \cong\left(\frac{\pi}{2 n}\right)^{1 / 2} \sigma e^{-n h(\hat{\psi})}
$$

Para o caso de dois parâmetros com o espaço paramétrico $\Psi=\left\{\left(\psi_{1}, \psi_{2}\right)\right.$, $\left.-\infty<\psi_{1}<a_{1},-\infty<\psi_{2}<a_{2}\right\}$, com $a_{1}$ e $a_{2}$ constantes temos

$$
I_{2}=\int_{-\infty}^{a_{2}} \int_{-\infty}^{a_{1}} e^{-n h\left(\psi_{1}, \psi_{2}\right)} d \psi_{1} d \psi_{2}
$$

Procedendo de modo análogo ao caso de um parâmetro, obtemos

$$
\hat{I}_{2} \cong(2 \pi) e^{-n h\left(\hat{\psi}_{1}, \hat{\psi}_{2}\right)}\left\{\operatorname{det}\left(n \Sigma_{h}^{-1}\left(\hat{\psi}_{1}, \hat{\psi}_{2}\right)\right)\right\}^{-1 / 2} F\left(a_{1}, a_{2}\right),
$$

onde $\left(\hat{\psi}_{1}, \hat{\psi}_{2}\right)$ maximizam $-h$ e $F\left(a_{1}, a_{2}\right)$ é a função de distribuição de uma normal bivariada com vetor de médias $\left(\hat{\psi}_{1}, \hat{\psi}_{2}\right)$ e matriz de variância e covariância $n \Sigma_{h}\left(\hat{\psi}_{1}, \hat{\psi}_{2}\right)$.

A aproximação em (2.13) pode ser simplificada quando os parâmetros $\psi_{1}$ e $\psi_{2}$ são ortogonais, com $\partial^{2} h\left(\hat{\psi}_{1}, \hat{\psi}_{2}\right) / \partial \psi_{1} \partial \psi_{2}=0$ (ver por exemplo, Cox e Reid, 1987). Nesse caso a aproximação em (2.13) é dada por

$$
\hat{I}_{2} \cong(2 \pi) e^{-n h\left(\hat{\psi}_{1}, \hat{\psi}_{2}\right)} \sigma_{1} \sigma_{2} \Phi\left(\frac{a_{1}-\hat{\psi}_{1}}{\sigma_{1}}\right) \Phi\left(\frac{a_{2}-\hat{\psi}_{2}}{\sigma_{2}}\right),
$$


onde $\sigma_{1}=\left\{\frac{n \partial^{2} h\left(\hat{\psi}_{1}, \hat{\psi}_{2}\right)}{\partial \psi_{1}^{2}}\right\}^{-1 / 2}$ e $\sigma_{2}=\left\{\frac{n \partial^{2} h\left(\hat{\psi}_{1}, \hat{\psi}_{2}\right)}{\partial \psi_{2}^{2}}\right\}^{-1 / 2}$.

Resultados similares são obtidos para outros casos; por exemplo, considerando $\Psi=\left\{\left(\psi_{1}, \psi_{2}\right),-\infty<\psi_{1}<a_{1},-\infty<\psi_{2}<\infty\right\}$, temos

$$
I_{3}=\int_{-\infty}^{\infty} \int_{-\infty}^{a_{1}} e^{-n h\left(\psi_{1}, \psi_{2}\right)} d \psi_{1} d \psi_{2}
$$

e a aproximação para $I_{3}$ é dada por

$$
\hat{I}_{3} \cong(2 \pi) e^{-n h\left(\hat{\psi}_{1}, \hat{\psi}_{2}\right)}\left\{\operatorname{det}\left(n \Sigma_{h}^{-1}\left(\hat{\psi}_{1}, \hat{\Psi}_{2}\right)\right)\right\}^{-1 / 2} \Phi\left(\frac{a_{1}-\hat{\psi}_{1}}{\sigma_{1}}\right)
$$

Também considerando, $\Psi=\left\{\left(\psi_{1}, \psi_{2}\right), a_{1}<\psi_{1}<\infty,-\infty<\psi_{2}<\infty\right\}$, temos

$$
\begin{aligned}
I_{4} & =\int_{-\infty}^{\infty} \int_{a_{1}}^{\infty} e^{-n h\left(\psi_{1}, \psi_{2}\right)} d \psi_{1} d \psi_{2} \\
& \cong(2 \pi) e^{-n h\left(\hat{\psi}_{1}, \hat{\psi}_{2}\right)}\left\{\operatorname{det}\left(n \Sigma_{h}^{-1}\left(\hat{\psi}_{1}, \hat{\psi}_{2}\right)\right)\right\}^{-1 / 2}\left\{1-\Phi\left(\frac{a_{1}-\hat{\psi}_{1}}{\sigma_{1}}\right)\right\}
\end{aligned}
$$

onde $\sigma_{1}=\left\{\frac{\partial^{2} h\left(\hat{\psi}_{1}, \hat{\psi}_{2}\right) / \partial \psi_{1}^{2}}{\Delta}\right\}^{-1 / 2}, \Delta=\left(\frac{n \partial^{2} h\left(\hat{\psi}_{1}, \hat{\psi}_{2}\right)}{\partial \psi_{1}^{2}}\right)\left(\frac{n \partial^{2} h\left(\hat{\psi}_{1}, \hat{\psi}_{2}\right)}{\partial \psi_{2}^{2}}\right)-\left(\frac{n \partial^{2} h\left(\hat{\psi}_{1}, \hat{\psi}_{2}\right)}{\partial \psi_{1} \partial \psi_{2}}\right)^{2}$ em $(2.15)$ e (2.16).

Por outro lado, se $\psi_{1}$ e $\psi_{2}$ são parâmetros ortogonais em (2.15) e (2.16) temos respectivamente 


$$
\begin{gathered}
\hat{I}_{3} \cong(2 \pi) e^{-n h\left(\hat{\psi}_{1}, \hat{\psi}_{2}\right)} \sigma_{1} \sigma_{2} \Phi\left(\frac{a_{1}-\hat{\psi}_{1}}{\sigma_{1}}\right), \\
\hat{I}_{4} \cong(2 \pi) e^{-n h\left(\hat{\psi}_{1}, \hat{\psi}_{2}\right)} \sigma_{1} \sigma_{2}\left\{1-\Phi\left(\frac{a_{1}-\hat{\psi}_{1}}{\sigma_{1}}\right)\right\} .
\end{gathered}
$$

Quando $-\infty<\psi_{1}<\infty, a_{2}<\psi_{2}<\infty$, temos

$$
\begin{aligned}
I_{5} & =\int_{a_{2}}^{\infty} \int_{-\infty}^{\infty} e^{-n h\left(\psi_{1}, \psi_{2}\right)} d \psi_{1} d \psi_{2} \\
& \cong(2 \pi) e^{-n h\left(\hat{\psi}_{1}, \hat{\psi}_{2}\right)} \sigma_{1} \sigma_{2}\left\{1-\Phi\left(\frac{a_{2}-\hat{\psi}_{2}}{\sigma_{2}}\right)\right\},
\end{aligned}
$$

onde $\sigma_{1}=\left\{\frac{\partial^{2} h\left(\hat{\psi}_{1}, \hat{\psi}_{2}\right)}{\partial \psi_{1}^{2}}\right\}^{-1 / 2}$ e $\sigma_{2}=\left\{\frac{\partial^{2} h\left(\hat{\psi}_{1}, \hat{\psi}_{2}\right)}{\partial \psi_{2}^{2}}\right\}^{-1 / 2}$ nas expressões (2.17), (2.18) e (2.19).

Para o caso multiparamétrico, com $\psi \in R^{m}$, os resultados obtidos são similares.

\subsection{Métodos Numéricos}

Outra estratégia bastante explorada entre os estatísticos Bayesianos são os métodos numéricos para aproximação de integrais (ver por exemplo, Naylor e Smith; 1982), Esses métodos são mais apropriados para problemas com número pequeno de parâmetros. Entre os vários métodos existentes na bibliografia, destacamos a seguir os que foram utilizados nesse trabalho. 


\subsubsection{Regra de Simpson}

Para o caso uniparamétrico a regra de Simpson é dada por

$$
\int_{a}^{b} f(\psi) d \psi \cong \frac{h}{3}\left(f_{0}+4 f_{1}+2 f_{2}+4 f_{3}+\ldots+2 f_{2 n-2}+4 f_{2 n-1}+f_{2 n}\right)
$$

onde $f_{r}=f\left(\psi_{r}\right)$, e o intervalo finito $[a, b]$ é dividido em $2 n$ (número par) subintervalos cada um de tamanho $h$, tal que $b-a=2 n h$. O erro de (2.20) é $R_{n}=-\frac{1}{180}(b-a) h^{4} f^{(4)}(\xi), a<\xi<b$.

A regra de Simpson é apropriada quando o intervalo $[a, b]$ é finito e $f(\psi)$ é diferenciável, o que nem sempre ocorre em inferência Bayesiana, ou mesmo, $f(\psi)$ pode ser uma função muito complicada.

\subsubsection{Regra Gaussiana}

Naylor e Smith (1982) usam o procedimento de quadratura Gaussiana para resolver problemas de integração em inferência Bayesiana.

Para utilizar essa metodologia, devemos escolher uma função integrável não negativa $\omega(\psi)$, tal que

$$
\int_{a}^{b} f(\psi) d \psi=\int_{a}^{b} g_{G}(\psi) \omega(\psi) d \psi
$$

onde $g_{G}(\psi)=f(\psi) / \omega(\psi)$.

Um resultado clássico em integração numérica é que se $P_{2 n-1}(\psi)$ representa um polinômio qualquer de grau $(2 n-1)$ em $\psi$ definido em $[a, b]$, e $f_{n}(\psi)$ é uma seqüência 
de polinômios ortogonais em $[a, b]$, relativo a uma função de peso positivo $\omega(\psi)$ tal que $f_{n}(\psi)$ seja um polinômio positivo, então

$$
\int_{a}^{b} \omega(\psi) P_{2 n-1}(\psi) d \psi=\sum_{k=1}^{n} \alpha_{k} P_{2 n-1}\left(\psi_{k}\right),
$$

onde $\alpha_{k}$ são os coeficientes e $\psi_{k}^{\prime}$ s as raízes de $f_{n}(\psi)=0$. Os coeficiente são dados por

$$
\alpha_{k}=\int_{a}^{b} \frac{\omega(\psi) f_{n}(\psi) d \psi}{\left(\psi-\psi_{k}\right) f^{\prime}\left(\psi_{k}\right)}
$$

Na prática, podemos usar diferentes sistemas de polinômios ortogonais. Uma possibilidade é a classe de polinômios relativos à função de peso $\omega(\psi)=\exp \left(-\psi^{2}\right)$ no intervalo $[a, b]=[-\infty, \infty]$.

Dessa forma, em (2.22) temos a aproximação

$$
\int_{-\infty}^{\infty} e^{-\psi^{2}} g_{G}(\psi) d \psi \cong \sum_{k=1}^{n} \alpha_{k} g_{G}\left(\psi_{k}\right)+R_{n}
$$

onde $\psi_{1}, \psi_{2}, \ldots, \psi_{n}$ são as raízes da equação polinomial de Hermite $H_{n}(\psi)=0$, e

$$
\alpha_{k}=\frac{2^{n-1} n ! \sqrt{\pi}}{n^{2}\left[H_{n-1}\left(\psi_{k}\right)\right]^{2}} .
$$

Os valores das raízes $\psi_{k}$ e os coeficientes $\alpha_{k}$ são dados em tabelas (ver por exemplo, Abramowitz e Stegun, 1965, p. 924). 


\section{Capítulo 3}

\section{Análise Bayesiana para o Modelo de Efeitos Aleatórios com 2 Componentes de Variância}

Os métodos Bayesianos constituem um conjunto de técnicas alternativas à análise clássica que podem facilitar a interpretação dos resultados e que permitem a incorporação de informações sobre os parâmetros do modelo antes de observar os dados. Esse conhecimento prévio é expressado através de uma densidade de probabilidade, conhecida como densidade a priori, a qual é atribuída aos parâmetros, que sob esse ponto de vista são considerados variáveis aleatórias. O Teorema de Bayes proporciona a obtenção de uma densidade a posteriori a qual combina a informação vinda dos dados, através da função de verossimilhança, com a informação prévia, representada na densidade a priori (ver por exemplo Box e Tiao, 1973).

Nesse capítulo apresentamos uma análise Bayesiana para o modelo de efeitos aleatórios com 2 componentes de variância (1.8), assumindo densidades a priori nãoinformativas para obter as densidades e momentos a posteriori para os parâmetros de interesse $\sigma_{1}^{2}$ e $\sigma_{2}^{2}$ e para algumas funções dos mesmos. Para isso, utilizamos métodos para aproximação de integrais, descritos no capítulo 2, para obter esses resultados quando não for possível obter a sua forma exata. Também, exploramos alguns aspectos 
de reparametrização a fim de melhorar a precisão dos resultados aproximados (ver por exemplo, Achcar e Smith, 1990).

\subsection{Densidades a Priori Não-Informativas para $\mu, \sigma_{1}^{2}$ e $\sigma_{2}^{2}$}

Nesse trabalho, vamos assumir que nenhum conhecimento prévio seja dado sobre os parâmetros no início do estudo. Nesse caso toda informação relevante aos parâmetros vem dos dados e portanto devemos formular uma densidade a priori nãoinformativa. Entre as possíveis formas para atribuir uma densidade a priori nãoinformativa, vamos considerar a regra de Jeffreys (ver por exemplo, Box e Tiao, 1973), a qual determina que uma densidade a priori não-informativa é dada por

$$
\pi\left(\mu, \sigma_{1}^{2}, \sigma_{2}^{2}\right) \propto\left|\operatorname{det} I\left(\mu, \sigma_{1}^{2}, \sigma_{2}^{2}\right)\right|^{1 / 2},
$$

onde $I\left(\mu, \sigma_{1}^{2}, \sigma_{2}^{2}\right)$ é a matriz de Informação de Fisher para $\mu, \sigma_{1}^{2}$ e $\sigma_{2}^{2}$ dada em (1.28). Assim,

$$
\operatorname{det} I\left(\mu, \sigma_{1}^{2}, \sigma_{2}^{2}\right)=\frac{J K^{3}\left(v_{2}+1\right) v_{1}}{4 \sigma_{1}^{4} \sigma_{12}^{6}}
$$

onde $\sigma_{12}^{2}=\sigma_{1}^{2}+K \sigma_{2}^{2}$ (como definido na tabela 1.3). Logo, em termos dos parâmetros $\left(\mu, \sigma_{1}^{2}, \sigma_{2}^{2}\right)$, a densidade a priori não-informativa, de acordo com (3.1) e (3.2) é dada por

$$
\pi\left(\mu, \sigma_{1}^{2}, \sigma_{2}^{2}\right) \propto \sigma_{1}^{-2}\left(\sigma_{1}^{2}+K \sigma_{2}^{2}\right)^{-3 / 2} .
$$


Outra densidade a priori que consideramos é a adotada por Box e Tiao (1973), os quais baseiam-se no fato que a função de verossimilhança (1.12) pode ser vista como sendo constituída por $J$ observações independentes de uma distribuição $N\left(\mu, \sigma_{12}^{2} / K\right)$ e $J(K-1)$ observações adicionais, independentes, de uma distribuição $N\left(0, \sigma_{1}^{2}\right)$. Box e Tiao tratam o parâmetro de locação, $\mu$, separadamente dos parâmetros $\left(\sigma_{1}^{2}, \sigma_{12}^{2}\right)$ e tomam como uma densidade priori de referência uma distribuição com $\left(\mu, \log \sigma_{1}^{2}, \log \sigma_{12}^{2}\right)$ localmente uniforme e localmente independente.

Desta maneira, uma distribuição a priori não-informativa para $\left(\mu, \sigma_{1}^{2}, \sigma_{12}^{2}\right)$ é dada por

$$
\pi\left(\mu, \sigma_{1}^{2}, \sigma_{12}^{2}\right)=\pi(\mu) \pi\left(\sigma_{1}^{2}, \sigma_{12}^{2}\right),
$$

com

$$
\begin{aligned}
& \pi(\mu) \propto \text { Constante e } \\
& \pi\left(\sigma_{1}^{2}, \sigma_{12}^{2}\right) \propto \sigma_{1}^{-2} \sigma_{12}^{-2} .
\end{aligned}
$$

Como é observado por Box e Tiao (1973), a densidade a priori para os parâmetros $\left(\sigma_{1}^{2}, \sigma_{12}^{2}\right)$ em (3.5), pode ser obtida diretamente utilizando a regra de Jeffeys. De acordo com (1.28), a matriz de informação para $\left(\sigma_{1}^{2}, \sigma_{12}^{2}\right)$ é dada por

$$
I\left(\sigma_{1}^{2}, \sigma_{12}^{2}\right)=\left[\begin{array}{cc}
\frac{v_{1}}{2\left(\sigma_{1}^{2}\right)^{2}}+\frac{\left(v_{2}+1\right)}{2\left(\sigma_{12}^{2}\right)^{2}} & \frac{K\left(v_{2}+1\right)}{2\left(\sigma_{12}^{2}\right)^{2}} \\
\frac{K\left(v_{2}+1\right)}{2\left(\sigma_{12}^{2}\right)^{2}} & \frac{K^{2}\left(v_{2}+1\right)}{2\left(\sigma_{12}^{2}\right)^{2}}
\end{array}\right] .
$$

O determinante da matriz $I\left(\sigma_{1}^{2}, \sigma_{12}^{2}\right)$ é dado por

$$
\operatorname{det} I\left(\sigma_{1}^{2}, \sigma_{12}^{2}\right)=\frac{K^{2}\left(v_{2}+1\right) v_{1}}{4 \sigma_{1}^{4} \sigma_{12}^{4}}
$$


e utilizando a regra de Jeffreys para obter a densidade a priori para $\left(\sigma_{1}^{2}, \sigma_{12}^{2}\right)$, ou seja, $\pi\left(\sigma_{1}^{2}, \sigma_{12}^{2}\right) \propto\left|\operatorname{det} I\left(\sigma_{1}^{2}, \sigma_{12}^{2}\right)\right|^{1 / 2}$, obtemos a mesma densidade a priori dada em (3.5).

Em termos dos parâmetros $\mu, \sigma_{1}^{2}$ e $\sigma_{2}^{2}$, e de acordo com (3.4) e (3.5), a densidade a priori não-informativa é dada por

$$
\pi\left(\mu, \sigma_{1}^{2}, \sigma_{2}^{2}\right) \propto \sigma_{1}^{-2}\left(\sigma_{1}^{2}+K \sigma_{2}^{2}\right)^{-1}
$$

onde $\sigma_{1}^{2}, \sigma_{2}^{2}>0$.

\subsection{Densidades a Posteriori Conjuntas para $\mu, \sigma_{1}^{2}$ e $\sigma_{2}^{2}$}

Combinando a função de verossimilhança (1.14) com a densidade a priori (3.3) (ver por exemplo, Press, 1989), obtemos a densidade a posteriori conjunta para $\mu, \sigma_{1}^{2}$ e $\sigma_{2}^{2}$ a qual é dada por

$$
\begin{aligned}
\pi\left(\mu, \sigma_{1}^{2}, \sigma_{2}^{2} / y\right) & \propto\left(\sigma_{1}^{2}\right)^{-\left(v_{1}+2\right) / 2}\left(\sigma_{1}^{2}+K \sigma_{2}^{2}\right)^{-\left(v_{2}+4\right) / 2} \times \\
& \times \exp \left\{-\frac{1}{2}\left[\frac{J K(y-\mu)^{2}}{\sigma_{1}^{2}+K \sigma_{2}^{2}}+\frac{v_{2} m_{2}}{\sigma_{1}^{2}+K \sigma_{2}^{2}}+\frac{v_{1} m_{1}}{\sigma_{1}^{2}}\right]\right\},
\end{aligned}
$$

onde $-\infty<\mu<\infty$ e $\sigma_{1}^{2}, \sigma_{2}^{2}>0$ e $y$ é um vetor de dados.

Do mesmo modo, considerando a densidade a priori (3.6), obtemos a densidade a posteriori conjunta para $\mu, \sigma_{1}^{2}$ e $\sigma_{2}^{2}$ dada por 


$$
\begin{aligned}
\pi\left(\mu, \sigma_{1}^{2}, \sigma_{2}^{2} / y\right) & \propto\left(\sigma_{1}^{2}\right)^{-\left(v_{1}+2\right) / 2}\left(\sigma_{1}^{2}+K \sigma_{2}^{2}\right)^{-\left(v_{2}+3\right) / 2} \times \\
& \times \exp \left\{-\frac{1}{2}\left[\frac{J K(y-\mu)^{2}}{\sigma_{1}^{2}+K \sigma_{2}^{2}}+\frac{v_{2} m_{2}}{\sigma_{1}^{2}+K \sigma_{2}^{2}}+\frac{v_{1} m_{1}}{\sigma_{1}^{2}}\right]\right\},
\end{aligned}
$$

onde $-\infty<\mu<\infty$ e $\sigma_{1}^{2}, \sigma_{2}^{2}>0$.

\subsubsection{Densidades a Posteriori Marginais Conjuntas para $\sigma_{1}^{2}$ e $\sigma_{2}^{2}$}

Como estamos interessados em inferências apenas nos parâmetros $\sigma_{1}^{2} \mathrm{e} \sigma_{2}^{2}$, é mais conveniente obtê-las a partir das densidades a posteriori marginais para esses parâmetros. A densidade a posteriori marginal conjunta para $\sigma_{1}^{2}$ e $\sigma_{2}^{2}$ é obtida integrando a densidade a posteriori conjunta para $\mu, \sigma_{1}^{2}$ e $\sigma_{2}^{2}$ com respeito a $\mu$ ou seja,

$$
\pi\left(\sigma_{1}^{2}, \sigma_{2}^{2} / y\right)=\int_{-\infty}^{\infty} \pi\left(\mu, \sigma_{1}^{2}, \sigma_{2}^{2} / y\right) d \mu
$$

Integrando (3.7) com respeito à $\mu$, obtemos a densidade a posteriori marginal conjunta para $\sigma_{1}^{2}$ e $\sigma_{2}^{2}$, considerando uma densidade priori de Jeffreys (priori (3.3), dada por

$$
\pi\left(\sigma_{1}^{2}, \sigma_{2}^{2} / y\right) \propto\left(\sigma_{1}^{2}\right)^{-\left(\frac{v_{1}}{2}+1\right)}\left(\sigma_{1}^{2}+K \sigma_{2}^{2}\right)^{-\left(\frac{v_{2}}{2}+\frac{3}{2}\right)} \exp \left\{-\frac{1}{2}\left[\frac{v_{2} m_{2}}{\sigma_{1}^{2}+K \sigma_{2}^{2}}+\frac{v_{1} m_{1}}{\sigma_{1}^{2}}\right]\right\} .
$$

Da mesma forma, integrando (3.8) com respeito à $\mu$, obtemos a densidade a posteriori marginal conjunta para $\sigma_{1}^{2}$ e $\sigma_{2}^{2}$, considerando a priori proposta por Box e Tiao (3.6), dada por 


$$
\pi\left(\sigma_{1}^{2}, \sigma_{2}^{2} / y\right) \propto\left(\sigma_{1}^{2}\right)^{-\left(\frac{\nu_{1}}{2}+1\right)}\left(\sigma_{1}^{2}+K \sigma_{2}^{2}\right)^{-\left(\frac{\nu_{2}}{2}+1\right)} \exp \left\{-\frac{1}{2}\left[\frac{v_{2} m_{2}}{\sigma_{1}^{2}+K \sigma_{2}^{2}}+\frac{v_{1} m_{1}}{\sigma_{1}^{2}}\right]\right\}
$$

onde $\sigma_{1}^{2}, \sigma_{2}^{2}>0$ em $(3.10)$ e (3.11).

\subsubsection{Densidades a Posteriori Marginais Aproximadas para $\sigma_{1}^{2} \mathrm{e}$ $\sigma_{2}^{2}$}

Em inferência Bayesiana, as inferências são tipicamente baseadas nas densidades a posteriori marginais dos parâmetros de interesse, nesse caso $\sigma_{1}^{2}$ e $\sigma_{2}^{2}$. As densidades a posteriori marginais para $\sigma_{1}^{2}$ e $\sigma_{2}^{2}$ são obtidas integrando a densidade a posteriori marginal conjunta (3.10) (ou também, 3.11) com respeito a $\sigma_{1}^{2}$ e $\sigma_{2}^{2}$ respectivamente. Uma vez que essas integrais não possuem soluções analíticas explícitas, utilizaremos o método do Laplace para aproximação de integrais, apresentado no capítulo 2 (ver por exemplo, Tierney e Kadane, 1986), para obter uma aproximação.

A densidade a posteriori marginal para $\sigma_{1}^{2}$, considerando a densidade a priori não-informativa (3.3), é dada por

$$
\pi\left(\sigma_{1}^{2} / y\right) \propto\left(\sigma_{1}^{2}\right)^{-\left(\frac{v_{1}}{2}+1\right)} \exp \left[-\frac{v_{1} m_{1}}{2 \sigma_{1}^{2}}\right] \int \exp \left[-n h_{\sigma_{1}^{2}}\left(\sigma_{2}^{2}\right)\right] d \sigma_{2}^{2},
$$

onde,

$$
-n h_{\sigma_{1}^{2}}\left(\sigma_{2}^{2}\right)=-\frac{\left(v_{2}+3\right)}{2} \ln \left(\sigma_{1}^{2}+K \sigma_{2}^{2}\right)-\frac{v_{2} m_{2}}{2\left(\sigma_{1}^{2}+K \sigma_{2}^{2}\right)}
$$

Portanto, utilizando o método de Laplace em (3.12) obtemos a densidade a posteriori marginal aproximada para $\sigma_{1}^{2}$, dada por 


$$
\pi\left(\sigma_{1}^{2} / y\right) \propto\left(\sigma_{1}^{2}\right)^{-\left(\frac{\nu_{1}}{2}+1\right)} \exp \left[-\frac{1}{\sigma_{1}^{2}}\left(\frac{v_{1} m_{1}}{2}\right)\right] .
$$

Observe que a densidade aproximada em (3.13) é uma densidade Gama invertida cuja moda a posteriori é dada por

$$
\tilde{\sigma}_{1}^{2}=\frac{v_{1} m_{1}}{2\left(v_{1} / 2+1\right)}
$$

Para calcular a moda basta derivar o logaritmo de (3.13) em relação a $\sigma_{1}^{2}$, igualar a zero e resolver em $\sigma_{1}^{2}$.

Similarmente, considerando a densidade a priori (3.6) e utilizando a método de Laplace, a densidade a posteriori marginal aproximada para $\sigma_{1}^{2}$ coincide com (3.13).

A densidade a posteriori marginal para $\sigma_{2}^{2}$ considerando a densidade a priori (3.3), pode ser escrita na forma:

$$
\pi\left(\sigma_{2}^{2} / y\right) \propto \int \exp \left[-n h_{\sigma_{2}^{2}}\left(\sigma_{1}^{2}\right)\right] d \sigma_{1}^{2}
$$

onde

$$
-n h_{\sigma_{2}^{2}}\left(\sigma_{1}^{2}\right)=-\frac{\left(v_{1}+2\right)}{2} \ln \left(\sigma_{1}^{2}\right)-\frac{\left(v_{2}+3\right)}{2} \ln \left(\sigma_{1}^{2}+K \sigma_{2}^{2}\right)-\frac{1}{2}\left[\frac{v_{2} m_{2}}{\sigma_{1}^{2}+K \sigma_{2}^{2}}+\frac{v_{1} m_{1}}{\sigma_{1}^{2}}\right] .
$$

Usando o método de Laplace em (3.15) obtemos uma expressão para a densidade a posteriori marginal aproximada para $\sigma_{2}^{2}$ dada por 


$$
\pi\left(\sigma_{2}^{2} / y\right) \propto \frac{\left(\hat{\sigma}_{1}^{2}+K \sigma_{2}^{2}\right)^{-\left(v_{2}+3\right) / 2} \exp \left\{-v_{2} m_{2} / 2\left(\hat{\sigma}_{1}^{2}+K \sigma_{2}^{2}\right)\right\}}{\left[\frac{v_{2} m_{2}}{\left(\hat{\sigma}_{1}^{2}+K \sigma_{2}^{2}\right)^{3}}+\frac{v_{1} m_{1}}{\left(\hat{\sigma}_{1}^{2}\right)^{3}}-\frac{\left(\dot{v}_{2}+3\right)}{2\left(\hat{\sigma}_{1}^{2}+K \sigma_{2}^{2}\right)^{2}}-\frac{\left(v_{1}+2\right)}{2\left(\hat{\sigma}_{1}^{2}\right)^{2}}\right]^{1 / 2}},
$$

onde $\sigma_{2}^{2}>0$ e $\hat{\sigma}_{1}^{2}$ maximiza $-n h_{\sigma_{2}^{2}}\left(\sigma_{1}^{2}\right)$ para cada valor de $\sigma_{2}^{2}$ fixo.

Da mesma forma, utilizando a densidade a priori não-informativa (3.6) e o método de Laplace, a densidade a posteriori marginal aproximada para $\sigma_{2}^{2}$ é dada por

$$
\pi\left(\sigma_{2}^{2} / y\right) \propto \frac{\left(\hat{\sigma}_{1}^{2}+K \sigma_{2}^{2}\right)^{-\left(v_{2}+2\right) / 2} \exp \left\{-v_{2} m_{2} / 2\left(\hat{\sigma}_{1}^{2}+K \sigma_{2}^{2}\right)\right\}}{\left[\frac{v_{2} m_{2}}{\left(\hat{\sigma}_{1}^{2}+K \sigma_{2}^{2}\right)^{3}}+\frac{v_{1} m_{1}}{\left(\hat{\sigma}_{1}^{2}\right)^{3}}-\frac{\left(v_{2}+2\right)}{2\left(\hat{\sigma}_{1}^{2}+K \sigma_{2}^{2}\right)^{2}}-\frac{\left(v_{1}+2\right)}{2\left(\hat{\sigma}_{1}^{2}\right)^{2}}\right]^{1 / 2}},
$$

onde $\sigma_{2}^{2}>0$ e $\hat{\sigma}_{1}^{2}$ maximiza $-n h_{\sigma_{2}^{2}}^{*}\left(\sigma_{1}^{2}\right)$ para cada valor de $\sigma_{2}^{2}$ fixo, com

$$
-n h_{\sigma_{2}^{2}}^{*}\left(\sigma_{1}^{2}\right)=-\frac{\left(v_{1}+2\right)}{2} \ln \left(\sigma_{1}^{2}\right)-\frac{\left(v_{2}+2\right)}{2} \ln \left(\sigma_{1}^{2}+K \sigma_{2}^{2}\right)-\frac{1}{2}\left[\frac{v_{2} m_{2}}{\sigma_{1}^{2}+K \sigma_{2}^{2}}+\frac{v_{1} m_{1}}{\sigma_{1}^{2}}\right] .
$$

As modas das densidades a posteriori marginais aproximadas (3.16) e (3.17) devem ser determinadas numericamente utilizando-se, por exemplo, o método iterativo de Newton-Raphson, pois não é possível obter uma solução analítica explícita para as mesmas. 


\subsubsection{Densidades a Posteriori Marginais para $\sigma_{2}^{2} / \sigma_{1}^{2}$}

Em problemas práticos pode surgir o interesse em conhecermos o comportamento de funções dos parâmetros e não apenas cada parâmetro separadamente. Um exemplo disso, é quando temos que distribuir um número fixo de unidades amostrais entre os dois estágios de amostragem, e nesse caso é mais útil conhecermos a razão de variâncias $\sigma_{2}^{2} / \sigma_{1}^{2}$ (ver por exemplo, Box, Hunter e Hunter, 1978). Por conveniência matemática trabalhamos com a razão $\sigma_{12}^{2} / \sigma_{1}^{2}$. Para encontrar a densidade a posteriori de interesse, consideramos a transformação de variáveis:

$$
W=\frac{\sigma_{12}^{2}}{\sigma_{1}^{2}}=1+K\left(\frac{\sigma_{2}^{2}}{\sigma_{1}^{2}}\right) \text { e } V=\sigma_{1}^{2}
$$

ou seja, $\sigma_{1}^{2}=V, \sigma_{2}^{2}=V(W-1) / K$. Dessa forma, o Jacobiano da transformação é dado por

$$
J(W, V)=\left|\begin{array}{ll}
\frac{\partial \sigma_{1}^{2}}{\partial W} & \frac{\partial \sigma_{1}^{2}}{\partial V} \\
\frac{\partial \sigma_{2}^{2}}{\partial W} & \frac{\partial \sigma_{2}^{2}}{\partial V}
\end{array}\right|=\left|\begin{array}{rr}
0 & 1 \\
\frac{V}{K} & -\frac{1}{K}
\end{array}\right| .
$$

Assim, $|J(W, V)|=V K^{-1}$ e a densidade a posteriori conjunta para $W$ e $V$, utilizando a regra de transformação de variáveis (ver por exemplo, Mood, Graybill e Boes, 1974) e de acordo com (3.10), é dada por

$$
\pi(w, v / y) \propto|J| \pi\left(\sigma_{1}^{2}, \sigma_{2}^{2} / y\right) .
$$

Isto é,

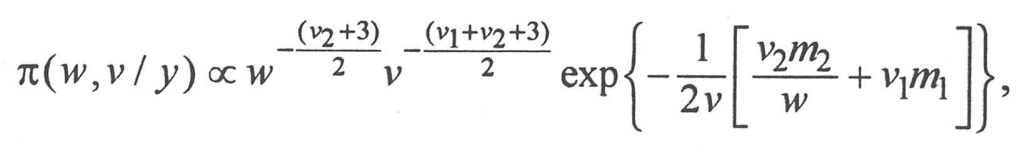

onde $w>1 \mathrm{e} v>0$. 
Várias integrais que aparecem nesse contexto podem ser facilmente calculadas com base no resultado baseado na distribuição Gama, dado a seguir (ver por exemplo, Box e Tiao, 1973, p. 145).

\section{Resultado 3.1}

$$
\int_{0}^{\infty} x^{-(p+1)} e^{-a x^{-1}} d x=a^{-p} \Gamma(p) .
$$

Integrando a densidade (3.18) com respeito a $V$, com base no resultado (3.1), obtemos a densidade a posteriori marginal para $W$, dada por

$$
\pi(w / y) \propto w^{-\frac{\left(v_{2}+3\right)}{2}}\left[\frac{v_{2} m_{2}}{w}+v_{1} m_{1}\right]^{-\frac{\left(v_{1}+v_{2}+1\right)}{2}},
$$

onde $w>1$.

A moda a posteriori de (3.19) é dada pelo valor

$$
\tilde{w}=\frac{v_{2} m_{2}\left(v_{1}-2\right)}{v_{1} m_{1}\left(v_{2}+3\right)} .
$$

Aplicando o mesmo procedimento, porém considerando a densidade a posteriori marginal conjunta (3.11), a densidade a posteriori marginal para $W$ é dada por

$$
\pi(w / y) \propto w^{-\frac{\left(v_{2}+2\right)}{2}}\left[\frac{v_{2} m_{2}}{w}+v_{1} m_{1}\right]^{-\frac{\left(v_{1}+v_{2}\right)}{2}},
$$


para $w>1$. A moda a posteriori, nesse caso, é dada por

$$
\tilde{w}=\frac{v_{2} m_{2}\left(v_{1}-2\right)}{v_{1} m_{1}\left(v_{2}+2\right)}
$$

\subsection{Densidade Preditiva para a Média $Y_{J+1}$ e de um Grupo Futuro}

A densidade preditiva para uma média $y_{J+1}$. de um grupo futuro de observações, dado um conjunto de dados $y$, é definida por

$$
\pi\left(y_{J+1} \cdot y\right)=\iiint f\left(y_{J+1} / \mu, \sigma_{1}^{2}, \sigma_{2}^{2}\right) \pi\left(\mu, \sigma_{1}^{2}, \sigma_{2}^{2} / y\right) d \mu d \sigma_{1}^{2} d \sigma_{2}^{2},
$$

onde $f\left(y_{J+1} \cdot / \mu, \sigma_{1}^{2}, \sigma_{2}^{2}\right)=\left(\frac{K}{2 \pi\left(\sigma_{1}^{2}+K \sigma_{2}^{2}\right)}\right)^{1 / 2} \exp \left\{-\frac{K\left(y_{J+1}-\mu\right)^{2}}{2\left(\sigma_{1}^{2}+K \sigma_{2}^{2}\right)}\right\}$ e $\pi\left(\mu, \sigma_{1}^{2}, \sigma_{2}^{2} / y\right)$ representa a densidade a posteriori conjunta (3.7) ou (3.8). Utilizando o seguinte resultado:

$$
v_{2} m_{2}+K\left(y_{. .}-\mu\right)^{2}+K\left(y_{J+1 .}-\mu\right)^{2}=v_{2} m_{2}+K\left(y_{J+1 .}-y_{. .}\right)^{2}+(J+1) K\left(y_{. .}-\mu\right)^{2},
$$

então, de acordo com a densidade a posteriori (3.7), temos

$$
\begin{aligned}
\pi\left(y_{J+1} \cdot / y\right) & \propto \iiint\left(\sigma_{1}^{2}\right)^{\frac{-\left(v_{1}+1\right)}{2}}\left(\sigma_{1}^{2}+K \sigma_{2}^{2}\right)^{\frac{-\left(v_{2}+5\right)}{2}} \times \\
& \times \exp \left\{-\frac{1}{2}\left[\frac{(J+1) K\left(y_{. .}-\mu\right)^{2}}{\sigma_{1}^{2}+K \sigma_{2}^{2}}+\frac{K\left(y_{J+1 \cdot}-y_{. .}\right)^{2}}{\sigma_{1}^{2}+K \sigma_{2}^{2}} \frac{v_{2} m_{2}}{\sigma_{1}^{2}+K \sigma_{2}^{2}}+\frac{v_{1} m_{1}}{\sigma_{1}^{2}}\right]\right\} d \mu d \sigma_{1}^{2} d \sigma_{2}^{2} .
\end{aligned}
$$


Integrando (3.23) com relação a $\mu$ obtemos

$$
\begin{aligned}
\pi\left(y_{J+1} . / y\right) & \left.\propto \iint\left(\sigma_{1}^{2}\right)^{\frac{-\left(v_{1}+1\right)}{2}} \sigma_{1}^{2}+K \sigma_{2}^{2}\right)^{\frac{-\left(v_{2}+4\right)}{2}} \times \\
& \times \exp \left\{-\frac{1}{2}\left[+\frac{K\left(y_{J+1}-y_{. .}\right)^{2}}{\sigma_{1}^{2}+K \sigma_{2}^{2}} \frac{v_{2} m_{2}}{\sigma_{1}^{2}+K \sigma_{2}^{2}}+\frac{v_{1} m_{1}}{\sigma_{1}^{2}}\right]\right\} d \sigma_{1}^{2} d \sigma_{2}^{2} .
\end{aligned}
$$

Utilizando o método de Laplace com $f=1$ em (2.3) (ver capítulo 2), obtemos uma aproximação para a densidade preditiva de $y_{J+1}$, de acordo com a densidade a posteriori (3.7), dada por

$$
\pi\left(y_{J+1} . / y\right) \propto\left[v_{2} m_{2}+K\left(y_{J+1}-y_{. .}\right)^{2}\right]^{-\left(\frac{v_{2}}{2}+1\right)} .
$$

De acordo com a densidade a posteriori (3.8) temos

$$
\pi\left(y_{J+1} \cdot / y\right) \propto\left[v_{2} m_{2}+K\left(y_{J+1}-y_{. .}\right)^{2}\right]^{-\left(\frac{v_{2}+1}{2}\right)},
$$

onde $-\infty<y_{J+1}<\infty, v_{2} m_{2}=S_{2}=K \sum_{j=1}^{J}\left(y_{j} .-y_{. .}\right)^{2}$ ( como definido na tabela 1.3), e com $y_{. .}=\frac{1}{(J+1)} \sum_{j=1}^{J+1} y_{j .}$ em (3.24) e (3.25). 


\subsection{Aproximação de Laplace para Momentos a Posteriori}

Nessa seção utilizamos o método de aproximação de Laplace para calcular momentos a posteriori dos parâmetros, e também de algumas funções dos mesmos. Com o objetivo de comparar a precisão dos resultados aproximados, na seção (3.5) serão obtidas as expressões exatas dos momentos a posteriori calculados aqui.

De acordo com a priori de Jeffeys (3.3), a média a posteriori para uma função positiva dos componentes de variância $g\left(\sigma_{1}^{2}, \sigma_{2}^{2}\right)$ pode ser escrita na forma:

$$
E\left\{g\left(\sigma_{1}^{2}, \sigma_{2}^{2}\right) / y\right\}=\frac{\iint \exp \left\{-n h^{*}\left(\sigma_{1}^{2}, \sigma_{2}^{2}\right)\right\} d \sigma_{1}^{2} d \sigma_{2}^{2}}{\iint \exp \left\{-n h\left(\sigma_{1}^{2}, \sigma_{2}^{2}\right)\right\} d \sigma_{1}^{2} d \sigma_{2}^{2}},
$$

onde

$$
\begin{gathered}
-n h^{*}\left(\sigma_{1}^{2}, \sigma_{2}^{2}\right)=\ln g\left(\sigma_{1}^{2}, \sigma_{2}^{2}\right)-n h\left(\sigma_{1}^{2}, \sigma_{2}^{2}\right) \mathrm{e} \\
-n h\left(\sigma_{1}^{2}, \sigma_{2}^{2}\right)=-\frac{\left(v_{1}+2\right)}{2} \ln \left(\sigma_{1}^{2}\right)-\frac{\left(v_{2}+3\right)}{2} \ln \left(\sigma_{1}^{2}+K \sigma_{2}^{2}\right)-\frac{1}{2}\left[\frac{v_{2} m_{2}}{\sigma_{1}^{2}+K \sigma_{2}^{2}}+\frac{v_{1} m_{1}}{\sigma_{1}^{2}}\right] .
\end{gathered}
$$

Usando o método de Laplace totalmente exponencial no numerador e denominador de (3.26) (ver capítulo 2), obtemos as aproximações:

$$
\begin{gathered}
\hat{E}\left(\sigma_{1}^{2} / y\right) \approx \frac{e\left(v_{1} m_{1}\right)\left(\frac{v_{1}}{2}\right)^{\frac{\left(v_{1}-3\right)}{2}}}{2\left(\frac{v_{1}}{2}+1\right)^{\frac{\left(v_{1}-1\right)}{2}}}, \\
\hat{E}\left\{\left(\sigma_{1}^{2}+K \sigma_{2}^{2}\right) / y\right\} \approx \frac{e\left(v_{2} m_{2}\right)\left(\frac{v_{2}}{2}+\frac{1}{2}\right)^{\frac{\left(v_{2}-2\right)}{2}}}{2\left(\frac{v_{2}}{2}+\frac{3}{2}\right)^{\frac{\left(v_{2}\right)}{2}}},
\end{gathered}
$$




$$
\begin{gathered}
\hat{E}\left\{\frac{\left(\sigma_{1}^{2}+K \sigma_{2}^{2}\right)}{\sigma_{1}^{2}} \mid y\right\} \approx \frac{\left(v_{2} m_{2}\right)\left(\frac{v_{1}}{2}+2\right)^{\frac{\left(v_{1}+1\right)}{2}}\left(\frac{v_{2}}{2}+\frac{1}{2}\right)^{\frac{\left(v_{2}-2\right)}{2}}}{\left(v_{1} m_{1}\right)\left(\frac{v_{1}}{2}+1\right)^{\frac{\left(v_{1}-1\right)}{2}}\left(\frac{v_{2}}{2}+\frac{3}{2}\right)^{\frac{\left(v_{2}\right)}{2}}}, \\
\hat{E}\left(\sigma_{2}^{2} / y\right) \approx \frac{K\left(v_{1} m_{1}\right)^{\frac{v_{1}}{2}}\left(v_{2} m_{2}\right)^{\frac{\left(v_{2}+1\right)}{2}} \exp \left\{\frac{\left(v_{1}+v_{2}\right)}{2}+\frac{5}{2}\right\} e^{-n h^{*}\left(\hat{\sigma}_{1}^{2}, \hat{\sigma}_{2}^{2}\right)}}{2^{\frac{\left(v_{1}+v_{2}+1\right)}{2}}\left(\frac{v_{1}}{2}+1\right)^{\frac{\left(v_{1}-1\right)}{2}}\left(\frac{v_{2}}{2}+\frac{3}{2}\right)^{\frac{v_{2}}{2}}\left\{\operatorname{det} n \Sigma_{h^{*}}\left(\hat{\sigma}_{1}^{2}, \hat{\sigma}_{2}^{2}\right)\right\}^{\frac{1}{2}}},
\end{gathered}
$$

onde $\hat{\sigma}_{1}^{2}$ e $\hat{\sigma}_{2}^{2}$ maximizam $-h^{*}$ e $\Sigma_{h^{*}}^{-1}\left(\hat{\sigma}_{1}^{2}, \hat{\sigma}_{2}^{2}\right)$ é a matriz Hessiana de $h^{*}$ calculada em $\hat{\sigma}_{1}^{2}$ e $\hat{\sigma}_{2}^{2}, \mathrm{com}$

$$
-n h^{*}\left(\sigma_{1}^{2}, \sigma_{2}^{2}\right)=\ln \left(\sigma_{2}^{2}\right)-\frac{\left(v_{1}+2\right)}{2} \ln \left(\sigma_{1}^{2}\right)-\frac{\left(v_{2}+3\right)}{2} \ln \left(\sigma_{1}^{2}+K \sigma_{2}^{2}\right)-\frac{1}{2}\left[\frac{v_{2} m_{2}}{\sigma_{1}^{2}+K \sigma_{2}^{2}}+\frac{v_{1} m_{1}}{\sigma_{1}^{2}}\right]
$$

Analogamente, é possível escrever a média a posteriori de uma função $g\left(\sigma_{1}^{2}, \sigma_{2}^{2}\right)$ de acordo com a priori de Box e Tiao (3.6), da mesma forma que em (3.26) redefinindo apenas

$$
-n h\left(\sigma_{1}^{2}, \sigma_{2}^{2}\right)=-\frac{\left(v_{1}+2\right)}{2} \ln \left(\sigma_{1}^{2}\right)-\frac{\left(v_{2}+2\right)}{2} \ln \left(\sigma_{1}^{2}+K \sigma_{2}^{2}\right)-\frac{1}{2}\left[\frac{v_{2} m_{2}}{\sigma_{1}^{2}+K \sigma_{2}^{2}}+\frac{v_{1} m_{1}}{\sigma_{1}^{2}}\right]
$$

A média a posteriori aproximada para $\sigma_{1}^{2}$ é igual para as duas densidades a priori consideradas. Os demais momentos a posteriori são dados por: 


$$
\begin{gathered}
\hat{E}\left(\left(\sigma_{1}^{2}+K \sigma_{2}^{2}\right) / y\right) \approx \frac{e\left(v_{2} m_{2}\right)\left(\frac{v_{2}}{2}\right)^{\frac{\left(v_{2}-3\right)}{2}}}{2\left(\frac{v_{2}}{2}+1\right)^{\frac{\left(v_{2}-1\right)}{2}}}, \\
\hat{E}\left\{\frac{\left(\sigma_{1}^{2}+K \sigma_{2}^{2}\right)}{\sigma_{1}^{2}} \mid y\right\} \approx \frac{\left(v_{2} m_{2}\right)\left(\frac{v_{1}}{2}+2\right)^{\frac{\left(v_{1}+1\right)}{2}}\left(\frac{v_{2}}{2}\right)^{\frac{\left(v_{2}-3\right)}{2}}}{\left(v_{1} m_{1}\right)\left(\frac{v_{1}}{2}+1\right)^{\frac{\left(v_{1}-1\right)}{2}}\left(\frac{v_{2}}{2}+1\right)^{\frac{\left(v_{2}-1\right)}{2}}}, \\
\hat{E}\left(\sigma_{2}^{2} / y\right) \approx \frac{K\left(v_{1} m_{1}\right)^{\frac{v_{1}}{2}}\left(v_{2} m_{2}\right)^{\frac{v_{2}}{2}} \exp \left\{\frac{\left(v_{1}+v_{2}\right)}{2}+2\right\} e^{-n h^{*}\left(\hat{\sigma}_{1}^{2}, \hat{\sigma}_{2}^{2}\right)}}{2^{\frac{\left(v_{1}+v_{2}\right)}{2}}\left(\frac{v_{1}}{2}+1\right)^{\frac{\left(v_{1}-1\right)}{2}}\left(\frac{v_{2}}{2}+1\right)^{\frac{\left(v_{2}-1\right)}{2}}\left\{\operatorname{det} n \Sigma_{h^{*}}\left(\hat{\sigma}_{1}^{2}, \hat{\sigma}_{2}^{2}\right)\right\}^{\frac{1}{2}}},
\end{gathered}
$$

onde $\hat{\sigma}_{1}^{2}$ e $\hat{\sigma}_{2}^{2}$ maximizam $-h^{*}$ em $(3.30)$ e $\Sigma_{h^{*}}^{-1}\left(\hat{\sigma}_{1}^{2}, \hat{\sigma}_{2}^{2}\right)$ é a matriz Hessiana de $h^{*}$ calculada em $\hat{\sigma}_{1}^{2}$ e $\hat{\sigma}_{2}^{2}$ e

$$
-n h^{*}\left(\sigma_{1}^{2}, \sigma_{2}^{2}\right)=\ln \left(\sigma_{2}^{2}\right)-\frac{\left(v_{1}+2\right)}{2} \ln \left(\sigma_{1}^{2}\right)-\frac{\left(v_{2}+2\right)}{2} \ln \left(\sigma_{1}^{2}+K \sigma_{2}^{2}\right)-\frac{1}{2}\left[\frac{v_{2} m_{2}}{\sigma_{1}^{2}+K \sigma_{2}^{2}}+\frac{v_{1} m_{1}}{\sigma_{1}^{2}}\right] .
$$

\subsubsection{Aproximação de Laplace Quando Ocorre o Problema de Estimativas Clássicas Negativas}

As aproximações de Laplace obtidas na seção anterior estão baseadas no fato que as funções envolvidas satisfazem certas condições de regularidades, como foi discutido no capítulo 2. No entanto, tais condições podem não estar sendo satisfeitas quando temos o problema de obtenção de estimativas negativas de $\sigma_{2}^{2}$ em (1.10). 
Observe que a moda da densidade a posteriori de (3.11), obtida calculando-se as derivadas parciais do logaritmo de (3.11), igualando a zero e resolvendo o sistema de equações em $\sigma_{1}^{2}$ e $\sigma_{2}^{2}$, é dada por

$$
\tilde{\sigma}_{1}^{2}=\frac{v_{1} m_{1}}{v_{1}+2}, \quad \tilde{\sigma}_{2}^{2}=\left(\frac{v_{2} m_{2}}{v_{2}+2}-\frac{v_{1} m_{1}}{v_{1}+2}\right) / K
$$

Se considerarmos que a moda deve estar sempre à direita da reta $\sigma_{2}^{2}=0$, então de (3.31) obtemos a relação,

$$
\frac{m_{2}}{m_{1}}>\frac{v_{1}\left(v_{2}+2\right)}{v_{2}\left(v_{1}+2\right)}
$$

Porém, quando $\hat{\sigma}_{2}^{2}<0$ (em 1.10) temos que $m_{2} / m_{1}<1$ e desde que $v_{1}>v_{2}$, teremos a desigualdade: $m_{2} / m_{1}<v_{1}\left(v_{2}+2\right) / v_{2}\left(v_{1}+2\right)$. Isso significa que o problema clássico de obtenção de estimativas negativas de $\sigma_{2}^{2}$ corresponde a uma distribuição a posteriori de $\sigma_{1}^{2}$ e $\sigma_{2}^{2}$ com a moda na reta $\sigma_{2}^{2}=0$, ou seja, a moda irrestrita em (3.31) leva a um valor negativo de $\sigma_{2}^{2}$, e portanto deve ser redefinida, onde chegamos à expressão,

$$
\tilde{\sigma}_{1}^{2}=\frac{v_{1} m_{1}+v_{2} m_{2}}{v_{1}+v_{2}+4}, \quad \tilde{\sigma}_{2}^{2}=0
$$

Quando esse tipo de problema ocorre, os máximos das função $-h^{*}\left(\sigma_{1}^{2}, \sigma_{2}^{2}\right)$ e $-h\left(\sigma_{1}^{2}, \sigma_{2}^{2}\right)$ em (3.27) estarão fora da região de definição de $\sigma_{1}^{2}$ e $\sigma_{2}^{2}$ e portanto as aproximações para os momentos a posteriori obtidas tornam-se inválidas. Para esse caso, podemos utilizar o método de Laplace considerando a restrição $\sigma_{2}^{2}>0$ proposto no capítulo 2 (seção 2.2).

Considere a aproximação de Laplace para o momento a posteriori na forma: 


$$
E\left\{g\left(\sigma_{1}^{2}, \sigma_{2}^{2}\right) / y\right\}=\frac{\int_{-\infty}^{\infty} \int_{0}^{\infty} \exp \left(-n h^{*}\left(\sigma_{1}^{2}, \sigma_{2}^{2}\right)\right) d \sigma_{1}^{2} d \sigma_{2}^{2}}{\int_{-\infty}^{\infty} \int_{0}^{\infty} \exp \left(-n h\left(\sigma_{1}^{2}, \sigma_{2}^{2}\right)\right) d \sigma_{1}^{2} d \sigma_{2}^{2}}
$$

com $-n h^{*}\left(\sigma_{1}^{2}, \sigma_{2}^{2}\right)$ e $-n h\left(\sigma_{1}^{2}, \sigma_{2}^{2}\right)$ definidas em (3.27). Aplicando o resultado (2.16) com $a_{1}=0$ ( ver capítulo 2) no numerador e denominador de (3.33), e considerando a densidade a posteriori (3.11), temos as seguintes aproximações para as médias a posteriori,

$$
\hat{E}\left(\sigma_{1}^{2} / y\right) \approx \frac{e\left(v_{1} m_{1}\right)\left(\frac{v_{1}}{2}\right)^{\frac{\left(v_{1}-3\right)}{2}}\left\{1-\Phi\left(-\frac{\hat{\sigma}_{2}^{* 2}}{\hat{v}_{2}^{*}}\right)\right\}}{2\left(\frac{v_{1}}{2}+1\right)^{\frac{\left(v_{1}-1\right)}{2}}\left\{1-\Phi\left(-\frac{\hat{\sigma}_{2}^{2}}{\hat{v}_{2}}\right)\right\}}
$$

onde $\hat{\sigma}_{2}^{* 2}=\left(\frac{v_{2} m_{2}}{v_{2}+2}-m_{1}\right) / K ; \hat{\sigma}_{2}^{2}=\left(\frac{v_{2} m_{2}}{v_{2}+2}-\frac{v_{1} m_{1}}{v_{1}+2}\right) / K$ é o valor de $\sigma_{2}^{2}$ que maximiza a função $-h$ no denominador e portanto é o mesmo para as demais expressões dadas a seguir, e $\hat{v}_{2}^{2}$ e $\hat{v}_{2}^{* 2}$ são as variâncias assintóticas de $\sigma_{2}^{2}$ de acordo com as funções $-n h\left(\sigma_{1}^{2}, \sigma_{2}^{2}\right)-n h^{*}\left(\sigma_{1}^{2}, \sigma_{2}^{2}\right)$ consideradas.

$$
\hat{E}\left(\left(\sigma_{1}^{2}+K \sigma_{2}^{2}\right) / y\right) \approx \frac{e\left(v_{2} m_{2}\right)\left(\frac{v_{2}}{2}\right)^{\frac{\left(v_{2}-3\right)}{2}}\left\{1-\Phi\left(-\frac{\hat{\sigma}_{2}^{* 2}}{\hat{v}_{2}^{*}}\right)\right\}}{2\left(\frac{v_{2}}{2}+1\right)^{\frac{\left(v_{2}-1\right)}{2}}\left\{1-\Phi\left(-\frac{\hat{\sigma}_{2}^{2}}{\hat{v}_{2}}\right)\right\}}
$$

$\operatorname{com} \hat{\sigma}_{2}^{* 2}=\left(m_{2}-\frac{v_{1} m_{1}}{v_{1}+2}\right) / K$ 
Também,

$$
\hat{E}\left\{\frac{\left(\sigma_{1}^{2}+K \sigma_{2}^{2}\right)}{\sigma_{1}^{2}} \mid y\right\} \approx \frac{\left(v_{2} m_{2}\right)\left(\frac{v_{1}}{2}+2\right)^{\frac{\left(v_{1}+1\right)}{2}}\left(\frac{v_{2}}{2}\right)^{\frac{\left(v_{2}-3\right)}{2}}\left\{1-\Phi\left(-\frac{\hat{\sigma}_{2}^{* 2}}{\hat{v}_{2}^{*}}\right)\right\}}{\left(v_{1} m_{1}\right)\left(\frac{v_{1}}{2}+1\right)^{\frac{\left(v_{1}-1\right)}{2}}\left(\frac{v_{2}}{2}+1\right)^{\frac{\left(v_{2}-1\right)}{2}}\left\{1-\Phi\left(-\frac{\hat{\sigma}_{2}^{2}}{\hat{v}_{2}}\right)\right\}},
$$

onde $\hat{\sigma}_{2}^{* 2}=\left(m_{2}-\frac{v_{1} m_{1}}{v_{1}+4}\right) / K$

\subsection{Fórmulas Exatas para os Momentos a Posteriori}

Para obter as fórmulas exatas dos momentos a posteriori dadas na seção (3.4) utilizamos o resultado a seguir, o que torna bastante simples os cálculos (ver por exemplo, Box e Tiao, 1973).

Resultado 3.2. Sejam as constantes $p_{1}>0, p_{2}>0, a_{1}>0, a_{2}>0$ e $c>0$; então temos:

$\int_{0}^{\infty} \int_{0}^{\infty} x^{-\left(p_{1}+1\right)}(x+c y)^{-\left(p_{2}+1\right)} \exp \left\{-\left(\frac{a_{1}}{x}+\frac{a_{2}}{x+c y}\right)\right\} d x d y=\frac{\Gamma\left(p_{1}\right) \Gamma\left(p_{2}\right)}{c a_{1}^{p_{1}} a_{2}^{p_{2}}} I_{a_{2} /\left(a_{1}+a_{2}\right)}\left(p_{2}, p_{1}\right)$,

onde $I_{x}(p, q)$ é a função Beta Incompleta (ver por exemplo, Abramowitz e Stegun, 1965).

Usando o resultado acima, podemos obter as constantes normalizadoras das densidades a posteriori marginais em (3.10) e (3.11). De a acordo com a priori de Jeffreys (3.3) e com o resultado (3.2), a densidade a posteriori marginal conjunta para $\left(\sigma_{1}^{2}, \sigma_{2}^{2}\right)$ é dada por 


$$
\begin{aligned}
\pi\left(\sigma_{1}^{2}, \sigma_{2}^{2} / y\right) & =\frac{K\left(v_{1} m_{1}\right)^{\frac{v_{1}}{2}}\left(v_{2} m_{2}\right)^{\frac{\left(v_{2}+1\right)}{2}}}{\Gamma\left(\frac{v_{1}}{2}\right) \Gamma\left(\frac{v_{2}}{2}+\frac{1}{2}\right)} I_{x}\left(\frac{v_{2}}{2}+\frac{1}{2} ; \frac{v_{1}}{2}\right) \times \\
& \times\left(\sigma_{1}^{2}\right)^{-\frac{\left(v_{1}+2\right)}{2}}\left(\sigma_{1}^{2}+K \sigma_{2}^{2}\right)^{-\frac{\left(v_{2}+3\right)}{2}} \exp \left\{-\frac{1}{2}\left[\frac{v_{2} m_{2}}{\sigma_{1}^{2}+K \sigma_{2}^{2}}+\frac{v_{1} m_{1}}{\sigma_{1}^{2}}\right]\right\} .
\end{aligned}
$$

Similarmente, utilizando a priori de Box e Tiao (3.6) e o resultado (3.2), a densidade a posteriori marginal conjunta para $\left(\sigma_{1}^{2}, \sigma_{2}^{2}\right)$ é dada por

$$
\begin{aligned}
\pi\left(\sigma_{1}^{2}, \sigma_{2}^{2} / y\right) & =\frac{K\left(v_{1} m_{1}\right)^{\frac{v_{1}}{2}}\left(v_{2} m_{2}\right)^{\frac{v_{2}}{2}}}{\Gamma\left(\frac{v_{1}}{2}\right) \Gamma\left(\frac{v_{2}}{2}\right)} I_{x}\left(\frac{v_{2}}{2} ; \frac{v_{1}}{2}\right) \times \\
& \times\left(\sigma_{1}^{2}\right)^{-\frac{\left(v_{1}+2\right)}{2}}\left(\sigma_{1}^{2}+K \sigma_{2}^{2}\right)^{-\frac{\left(v_{2}+2\right)}{2}} \exp \left\{-\frac{1}{2}\left[\frac{v_{2} m_{2}}{\sigma_{1}^{2}+K \sigma_{2}^{2}}+\frac{v_{1} m_{1}}{\sigma_{1}^{2}}\right]\right\},
\end{aligned}
$$

onde $\sigma_{1}^{2}>0, \sigma_{2}^{2}>0$ e $x=\frac{v_{2} m_{2}}{v_{1} m_{1}+v_{2} m_{2}}$ nas expressões (3.35) e (3.36).

Portanto, a média a posteriori de $\sigma_{1}^{2}$ de acordo com a densidade a priori (3.3), é dada por

$$
\begin{aligned}
& E\left(\sigma_{1}^{2} / y\right)=\frac{K\left(v_{1} m_{1}\right)^{\frac{v_{1}}{2}}\left(v_{2} m_{2}\right)^{\frac{\left(v_{2}+1\right)}{2}}}{\Gamma\left(\frac{v_{1}}{2}\right) \Gamma\left(\frac{v_{2}}{2}+\frac{1}{2}\right)} I_{x}\left(\frac{v_{2}}{2}+\frac{1}{2} ; \frac{v_{1}}{2}\right) \times \\
& \quad \times \int_{0}^{\infty} \int_{0}^{\infty}\left(\sigma_{1}^{2}\right)^{-\frac{v_{1}}{2}}\left(\sigma_{1}^{2}+K \sigma_{2}^{2}\right)^{-\frac{\left(v_{2}+3\right)}{2}} \exp \left\{-\frac{1}{2}\left[\frac{v_{2} m_{2}}{\sigma_{1}^{2}+K \sigma_{2}^{2}}+\frac{v_{1} m_{1}}{\sigma_{1}^{2}}\right]\right\} .
\end{aligned}
$$


Utilizando o resultado (3.2), temos

$$
E\left(\sigma_{1}^{2} / y\right)=\frac{v_{1} m_{1} I_{x}\left(\frac{v_{2}}{2}+\frac{1}{2} ; \frac{v_{1}}{2}-1\right)}{2\left(\frac{v_{1}}{2}-1\right) I_{x}\left(\frac{v_{2}}{2}+\frac{1}{2} ; \frac{v_{1}}{2}\right)}
$$

Procedendo de maneira semelhante é possível obter os demais momentos a posteriori calculados na seção anterior. De acordo com a priori (3.3) esses momentos são dados por:

$$
\begin{gathered}
E\left(\left(\sigma_{1}^{2}+K \sigma_{2}^{2}\right) / y\right)=\frac{v_{2} m_{2} I_{x}\left(\frac{v_{2}}{2}-\frac{1}{2} ; \frac{v_{1}}{2}\right)}{\left(v_{2}-1\right) I_{x}\left(\frac{v_{2}}{2}+\frac{1}{2} ; \frac{v_{1}}{2}\right)}, \\
E\left(\frac{\left(\sigma_{1}^{2}+K \sigma_{2}^{2}\right)}{\sigma_{1}^{2}} \mid y\right)=\frac{v_{2} m_{2} I_{x}\left(\frac{v_{2}}{2}-\frac{1}{2} ; \frac{v_{1}}{2}+1\right)}{\left(v_{2}-1\right) m_{1} I_{x}\left(\frac{v_{2}}{2}+\frac{1}{2} ; \frac{v_{1}}{2}\right)}, \\
E\left(\sigma_{2}^{2} / y\right)=\frac{v_{2} m_{2} I_{x}\left(\frac{v_{2}}{2}-\frac{1}{2} ; \frac{v_{1}}{2}\right)}{K\left(v_{2}-1\right) I_{x}\left(\frac{v_{2}}{2}+\frac{1}{2} ; \frac{v_{1}}{2}\right)}-\frac{v_{1} m_{1} I_{x}\left(\frac{v_{2}}{2}+\frac{1}{2} ; \frac{v_{1}}{2}-1\right)}{2 K\left(\frac{v_{1}}{2}-1\right) I_{x}\left(\frac{v_{2}}{2}+\frac{1}{2} ; \frac{v_{1}}{2}\right)} .
\end{gathered}
$$

Similarmente, considerando a densidade a priori (3.6) e o resultado (3.2), obtemos:

$$
E\left(\sigma_{1}^{2} / y\right)=\frac{v_{1} m_{1} I_{x}\left(\frac{v_{2}}{2} ; \frac{v_{1}}{2}-1\right)}{2\left(\frac{v_{1}}{2}-1\right) I_{x}\left(\frac{v_{2}}{2} ; \frac{v_{1}}{2}\right)},
$$




$$
\begin{gathered}
E\left(\left(\sigma_{1}^{2}+K \sigma_{2}^{2}\right) / y\right)=\frac{v_{2} m_{2} I_{x}\left(\frac{v_{2}}{2}-1 ; \frac{v_{1}}{2}\right)}{2\left(\frac{v_{2}}{2}-1\right) I_{x}\left(\frac{v_{2}}{2} ; \frac{v_{1}}{2}\right)}, \\
\hat{E}\left\{\frac{\left(\sigma_{1}^{2}+K \sigma_{2}^{2}\right)}{\sigma_{1}^{2}} \mid y\right\}=\frac{v_{2} m_{2} I_{x}\left(\frac{v_{2}}{2}-1 ; \frac{v_{1}}{2}+1\right)}{\left(v_{2}-2\right) m_{1} I_{x}\left(\frac{v_{2}}{2} ; \frac{v_{1}}{2}\right)}, \\
E\left(\sigma_{2}^{2} / y\right)=\frac{v_{2} m_{2} I_{x}\left(\frac{v_{2}}{2}-1 ; \frac{v_{1}}{2}\right)}{2 K\left(\frac{v_{2}}{2}-1\right) I_{x}\left(\frac{v_{2}}{2} ; \frac{v_{1}}{2}\right)}-\frac{v_{1} m_{1} I_{x}\left(\frac{v_{2}}{2} ; \frac{v_{1}}{2}-1\right)}{2 K\left(\frac{v_{1}}{2}-1\right) I_{x}\left(\frac{v_{2}}{2} ; \frac{v_{1}}{2}\right)} .
\end{gathered}
$$

Podemos observar que apesar de ser possível obter as expressões exatas para esses momentos a posteriori, essas fórmulas envolvem o uso da função Beta incompleta, e portanto o uso do método de Laplace pode ser de grande interesse prático.

\subsection{Reparametrização Logarítmica em $\sigma_{1}^{2}$ e $\sigma_{12}^{2}$}

Ao utilizarmos um método de aproximação de integrais, devemos estar cientes da precisão dos valores aproximados. Vários aspectos de inferência Bayesiana aproximada são apresentados por Achcar e Smith (1990), os quais observam que em geral, as aproximações de Laplace para integrais são mais precisas quando consideramos uma parametrização com priori não-informativa de Jeffreys localmente uniforme (ver por exemplo, Box e Tiao. 1973).

Observamos que as densidades a priori não-informativas (3.3) e (3.6) não são localmente uniformes (ver por exemplo, Box e Tiao, 1973), o que pode implicar em pouca precisão nas aproximações obtidas, especialmente para amostras moderadas ou pequenas.

Por outro lado, como já foi dito anteriormente, a densidade a priori (3.6) é obtida a partir de uma distribuição $\operatorname{com}\left(\mu, \ln \sigma_{1}^{2}, \ln \sigma_{12}^{2}\right)$ localmente uniforme e 
localmente independente (ver por exemplo, Box e Tiao, 1973). Portanto, se tomarmos a densidade a priori não-informativa (3.6), e assumirmos a parametrização $\mu=\mu$, $\xi_{1}=\ln \left(\sigma_{1}^{2}\right)$ e $\xi_{2}=K^{-1} \ln \left(\sigma_{1}^{2}+K \sigma_{2}^{2}\right)$, ou seja, $\sigma_{1}^{2}=e^{\xi_{1}}$ e $\sigma_{2}^{2}=K^{-1}\left(e^{K \xi_{2}}-e^{\xi_{1}}\right)$, a densidade a priori para $\left(\mu, \xi_{1}, \xi_{2}\right)$ é dada por

$$
\pi\left(\mu, \xi_{1}, \xi_{2}\right) \propto \text { Constante. }
$$

Assim, na parametrização $\left(\mu, \xi_{1}, \xi_{2}\right)$, temos uma densidade localmente uniforme e portanto, obtemos uma métrica paramétrica que só muda em locação e não em escala.

A função de verosimilhança em $\left(\mu, \xi_{1}, \xi_{2}\right)$ de acordo com (1.14) é dada por

$$
L\left(\mu, \xi_{1}, \xi_{2} / y\right) \propto\left(e^{\xi_{1}}\right)^{-\frac{v_{1}}{2}}\left(e^{K \xi_{2}}\right)^{-\frac{\left(v_{2}+1\right)}{2}} \exp \left\{-\frac{1}{2}\left[\frac{J K\left(y_{. .}-\mu\right)^{2}}{e^{K \xi_{2}}}+\frac{v_{2} m_{2}}{e^{K \xi_{2}}}+\frac{v_{1} m_{1}}{e^{\xi_{1}}}\right]\right\} .
$$

Combinando a função de verossimilhança (3.40) e a densidade a priori (3.39), obtemos a densidade a posteriori conjunta para $\left(\mu, \xi_{1}, \xi_{2}\right)$, dada por

$$
\pi\left(\mu, \xi_{1}, \xi_{2} / y\right) \propto\left(e^{\xi_{1}}\right)^{-\frac{v_{1}}{2}}\left(e^{K \xi_{2}}\right)^{-\frac{\left(v_{2}+1\right)}{2}} \exp \left\{-\frac{1}{2}\left[\frac{J K(y . . \mu)^{2}}{e^{K \xi_{2}}}+\frac{v_{2} m_{2}}{e^{K \xi_{2}}}+\frac{v_{1} m_{1}}{e^{\xi_{1}}}\right]\right\},
$$

onde $-\infty<\mu<\infty$ e $-\infty<\xi_{1}, \xi_{2}<\infty$.

A densidade a posteriori marginal conjunta para $\left(\xi_{1}, \xi_{2}\right)$ é obtida integrando (3.41) em relação a $\mu$, de onde obtemos a expressão:

$$
\pi\left(\xi_{1}, \xi_{2} / y\right) \propto\left(e^{\xi_{1}}\right)^{-\frac{v_{1}}{2}}\left(e^{K \xi_{2}}\right)^{-\frac{v_{2}}{2}} \exp \left\{-\frac{1}{2}\left[\frac{v_{2} m_{2}}{e^{K \xi_{2}}}+\frac{v_{1} m_{1}}{e^{\xi_{1}}}\right]\right\},
$$

onde $-\infty<\xi_{1}, \xi_{2}<\infty$ 
A seguir apresentamos a aproximação de Laplace na parametrização $\left(\xi_{1}, \xi_{2}\right)$, para os momentos a posteriori calculados nas seções (3.4) e (3.5).

\subsubsection{Aproximação de Laplace para Momentos a Posteriori} Considerando a Parametrização $\quad \xi_{1}=\ln \left(\sigma_{1}^{2}\right) \quad$ e $\xi_{2}=K^{-1} \ln \left(\sigma_{1}^{2}+K \sigma_{2}^{2}\right)$

A média a posteriori de uma função $g\left(\xi_{1}, \xi_{2}\right)$, de acordo com a parametrização $\left(\xi_{1}, \xi_{2}\right)$, pode ser escrita na forma:

$$
E\left(g\left(\xi_{1}, \xi_{2}\right) / y\right)=\frac{\iint \exp \left\{-n h^{*}\left(\xi_{1}, \xi_{2}\right)\right\} d \xi_{1} d \xi_{2}}{\iint \exp \left\{n h\left(\xi_{1}, \xi_{2}\right)\right\} d \xi_{1} d \xi_{2}}
$$

onde

$$
\begin{gathered}
-n h^{*}\left(\xi_{1}, \xi_{2}\right)=\ln g\left(\xi_{1}, \xi_{2}\right)-n h\left(\xi_{1}, \xi_{2}\right) \mathrm{e} \\
-n h\left(\xi_{1}, \xi_{2}\right)=-\frac{\left(v_{1}\right)}{2} \xi_{1}-\frac{\left(v_{2}\right)}{2} K \xi_{2}-\frac{1}{2}\left[\frac{v_{2} m_{2}}{e^{K \xi_{2}}}+\frac{v_{1} m_{1}}{e^{\xi_{1}}}\right]
\end{gathered}
$$

Utilizando o método de Laplace no numerador e denominador de (3.43) (ver por exemplo, Tierney e Kadane, 1986; capítulo 2), obtemos as seguintes aproximações:

$$
\hat{E}\left(\sigma_{1}^{2} / y\right) \approx \frac{e\left(v_{1} m_{1}\right)\left(\frac{v_{1}}{2}-1\right)^{\frac{\left(v_{1}-3\right)}{2}}}{2\left(\frac{v_{1}}{2}\right)^{\frac{\left(\nu_{1}-1\right)}{2}}}
$$




$$
\begin{gathered}
\hat{E}\left(\left(\sigma_{1}^{2}+K \sigma_{2}^{2}\right) / y\right) \approx \frac{e\left(v_{2} m_{2}\right)\left(\frac{v_{2}}{2}-1\right)^{\frac{\left(v_{1}-3\right)}{2}}}{2\left(\frac{v_{2}}{2}\right)^{\frac{\left(v_{2}-1\right)}{2}}}, \\
\hat{E}\left\{\frac{\left(\sigma_{1}^{2}+K \sigma_{2}^{2}\right)}{\sigma_{1}^{2}} \mid y\right\} \approx \frac{\left(v_{2} m_{2}\right)\left(\frac{v_{1}}{2}+1\right)^{\frac{\left(v_{1}+1\right)}{2}}\left(\frac{v_{2}}{2}-1\right)^{\frac{\left(v_{2}-3\right)}{2}}}{\left(v_{1} m_{1}\right)\left(\frac{v_{1}}{2}\right)^{\frac{\left(v_{1}-1\right)}{2}}\left(\frac{v_{2}}{2}\right)^{\frac{\left(v_{2}-1\right)}{2}}}, \\
\hat{E}\left(\sigma_{2}^{2} / y\right) \approx \frac{K\left(v_{1} m_{1}\right)^{\frac{v_{1}}{2}}\left(v_{2} m_{2}\right)^{\frac{v_{2}}{2}} \exp \left\{\frac{\left(v_{1}+v_{2}\right)}{2}\right\} e^{-n h^{*}\left(\hat{\xi}_{1}, \hat{\xi}_{2}\right)}}{2^{\frac{\left(v_{1}+v_{2}\right)}{2}}\left(\frac{v_{1}}{2}\right)^{\left(v_{1}-1\right) / 2}\left(\frac{v_{2}}{2}\right)^{\frac{\left(v_{2}-1\right)}{2}}\left\{\operatorname{det} n \Sigma_{h^{*}}^{-1}\left(\hat{\xi}_{1}, \hat{\xi}_{2}\right)\right\}^{\frac{1}{2}}},
\end{gathered}
$$

onde $\left(\hat{\xi}_{1}, \hat{\xi}_{2}\right)$ maximizam $-n h^{*}\left(\xi_{1}, \xi_{2}\right)$ e $\Sigma_{h^{*}}^{-1}\left(\hat{\xi}_{1}, \hat{\xi}_{2}\right)$ é a matriz Hessiana de $h^{*}$ calculada em $\left(\hat{\xi}_{1}, \hat{\xi}_{2}\right)$, com $-n h^{*}\left(\xi_{1}, \xi_{2}\right)$ dada por

$$
-n h^{*}\left(\xi_{1}, \xi_{2}\right)=-\ln K+\ln \left(e^{K \xi_{2}}-e^{\xi_{1}}\right)-\frac{\left(v_{1}\right)}{2} \xi_{1}-\frac{\left(v_{2}\right)}{2} K \xi_{2}-\frac{1}{2}\left[\frac{v_{2} m_{2}}{e^{K \xi_{2}}}+\frac{v_{1} m_{1}}{e^{\xi_{1}}}\right]
$$

Para o caso onde temos que calcular o método de aproximação de Laplace com restrição (ver seção 3.4.1), essa reparametrização pode são ser a mais adequada, pois a restrição $\sigma_{2}^{2}>0$ implica, em termos de $\left(\xi_{1}, \xi_{2}\right)$, em $\xi_{2}>\xi_{1} / K$, o que dificulta a obtenção dos percentis normais exigidos pelo método (ver capítulo 2, seção (2.2)). Para esse problema exploramos a parametrização do tipo: $\xi_{1}=\ln \left(\sigma_{1}^{2}\right)$, e $\xi_{2}$ dado pela transformação de Box e Cox (1964), ou seja, 


$$
\begin{aligned}
& \xi_{1}=\ln \left(\sigma_{1}^{2}\right), \\
& \xi_{2}=\left\{\begin{array}{ll}
\frac{\left(\sigma_{2}^{2}+3\right)^{\lambda}-1}{\lambda} & \text { se } \lambda \neq 0 \\
\ln \left(\sigma_{2}^{2}+3\right) & \text { se } \lambda=0
\end{array} .\right.
\end{aligned}
$$

O efeito da reparametrização pode ser avaliado comparando-se os resultados aproximados com o valor exato, já que nesse trabalho os mesmos são obtidos. Quando não existe esta possibilidade, uma maneira de avaliar o efeito da parametrização nas aproximações de Laplace é verificar a normalidade das densidades a posteriori envolvidas. Na próxima seção apresentamos algumas técnicas utilizadas neste trabalho.

\subsection{Medidas para Diagnóstico de Normalidade}

Em inferência Bayesiana um método diagnóstico de normalidade pode ser muito útil para verificar o quanto estamos melhorando a normalidade de uma distribuição a posteriori com uma reparametrização. Também na inferência clássica é importante verificar a normalidade da função de verossimilhança, para nos certificarmos que os resultados baseados na normalidade assintótica estejam condizentes com a realidade.

Uma maneira intuitiva de deduzir a normalidade das função de interesse, é verificar se seus gráficos apresentam simetria, no caso univariado, ou se os contornos apresentam formas de elipses aproximadas, no caso bivariado. Quando temos interesse num dado parâmetro $\theta_{j}$ e existem vários parâmetros auxiliares (parâmetros "nuisance") podemos verificar a normalidade através do gráfico da densidade a posteriori "profile" de $\theta_{j}$, dada por

$$
\pi\left(\theta_{j} / y\right)=\max _{\theta_{1}, \cdots, \theta_{j-1}, \theta_{j+1}, \cdots, \theta_{p}} \pi(\theta / y)
$$


Existem porém, outras técnicas que podem simplificar e tornar mais preciso o diagnóstico de normalidade. A seguir destacamos algumas dessas técnicas utilizadas nesse trabalho.

\subsubsection{T-plot de Hills e Smith}

Hills e Smith (1993) introduziram um método gráfico bastante prático para se verificar a normalidade de densidades a posteriori, o qual é uma generalização e extensão de um método proposto por Bates e Watts (1990).

Seja $\pi(\tilde{\theta} / y)$ o valor da densidade a posteriori calculada na moda $\left(\tilde{\theta}_{1}, \tilde{\theta}_{2}, \ldots, \tilde{\theta}_{p}\right)$, e seja $\pi\left(\theta_{j} / y\right)$ a densidade a posteriori "profile" de $\theta_{j}$ em (3.46). Uma medida de diagnóstico é dada por

$$
T^{*}\left(\theta_{j}\right)=\operatorname{sgn}\left(\theta_{j}-\tilde{\theta}_{j}\right)\left[-2 \ln \left\{\frac{\pi\left(\theta_{j} / y\right)}{\pi(\tilde{\theta} / y)}\right\}\right]^{1 / 2},
$$

onde $\operatorname{sgn}(x)$ é a função sinal de $x$.

O gráfico de $T^{*}\left(\theta_{j}\right)$ versus $\theta_{j}$ deverá ser uma linha reta caso o parâmetro seja normalmente distribuído e portanto, o gráfico pode ser usado para indicar a falta de normalidade.

Para o caso unidimensional, de acordo com (3.47), o T-plot de Hills e Smith é definido por

$$
T^{*}(\theta)=\operatorname{sgn}(\theta-\widetilde{\theta})\left[-2 \ln \left\{\frac{\pi(\theta / y)}{\pi(\tilde{\theta} / y)}\right\}\right]^{1 / 2} .
$$


O caso bidimensional é mais complexo, pois a extensão direta de (3.48) não funciona (ver por exemplo, Hills e Smith, 1993). Uma possibilidade é aplicar uma expansão em Série de Taylor para $\ln \left[\pi\left(\theta_{1}, \theta_{2} / y\right)\right]$ em torno da moda $\left(\tilde{\theta}_{1}, \tilde{\theta}_{2}\right)$, e construir os contornos $T^{*}\left(\theta_{1}, \theta_{2}\right)$ versus $\theta_{1}$ e $\theta_{2}$. Esse contorno deverá ter forma aproximadamente elíptica se os parâmetros são normalmente distribuídos. Entretanto essa estratégia é pouco viável, pois poderíamos analisar diretamente os contornos das densidades a posteriori. Uma alternativa, utilizada nesse trabalho, é fixar o valor de um dos parâmetros e analisar o comportamento do outro separadamente, e julgar o grau de linearidade do gráfico proposto por Hills e Smith.

\subsubsection{Terceira Derivada Padronizada}

A terceira derivada padronizada, proposta por Kass e Slate (1992), é uma medida de não-normalidade da função de verossimilhança ou de densidades a posteriori. Essa medida facilita as interpretações pois reduz as análises a um simples escalar. A terceira derivada padronizada foi originalmente proposta por Sprott (1973), a qual é dada por

$$
\operatorname{STD}(\hat{\theta})=\left|l^{\prime \prime \prime}(\hat{\theta}) \cdot\left[l^{\prime \prime}(\hat{\theta})\right]^{-3 / 2}\right|
$$

onde $l^{\prime \prime}(\hat{\theta})$ e $l^{\prime \prime \prime}(\hat{\theta})$ são as segundas e terceiras derivadas do logaritmo da função de verossimilhança calculadas no estimador de máxima verossimilhança $\hat{\theta}$. Se a função de verossimilhança estiver próxima da normal, a medida em (3.49) deve estar próxima ou ser igual a zero. De forma análoga podemos calcular a terceira derivada padronizada para a densidade a posteriori $\pi(\theta / y)$, substituindo o estimador de máxima verossimilhança $\hat{\theta}$ pela moda a posteriori $\tilde{\theta}$.

Para o caso multiparamétrico, podemos utilizar uma generalização da terceira derivada padronizada proposta por Kass e Slate (1992), para avaliar a normalidade conjunta da densidade a posteriori definida por 


$$
m^{2} \bar{B}^{2}=\sum_{i, j, k, l, m, n} b_{i j} b_{l m} b_{k n} d_{i j k} d_{l n m}
$$

onde $d_{i j k}=\frac{\partial^{3} \ln \pi(\tilde{\theta} / y)}{\partial \theta_{i} \partial \theta_{j} \partial \theta_{k}}, b_{i j}$ é o $i$ - $j$-ésimo elemento da matriz inversa de segundas derivadas do logaritmo da densidade a posteriori, calculada na moda, a menos do sinal e $m$ é o número de parâmetros.

Se a distribuição conjunta dos parâmetros estiver próxima da normal, então $m^{2} \bar{B}^{2}$ em (3.50) deve ser aproximadamente igual a zero.

Nesse trabalho utilizamos a generalização da terceira derivada padronizada (3.50) para avaliar a normalidade das densidades a posteriori (3.10) e (3.11), e também para encontrar um valor de $\lambda$ que forneça boa normalidade para a densidade a posteriori conjunta para $\xi_{1}$ e $\xi_{2}$ em (3.45), ou seja, procuramos um valor de $\lambda$ tal que $m^{2} \bar{B}^{2}$ seja próximo de zero. 


\section{Capítulo 4}

\section{Análise Bayesiana para o Modelo de Efeitos Aleatórios com 3 Componentes de Variância}

Consideremos o modelo com três componentes de variância (1.3) discutido inicialmente no capítulo 1 . Como já foi dito, esse modelo pode ser visto como uma extensão do modelo (1.8), adicionando-se ao mesmo outra fonte de variação. Nesse caso, um planejamento de classificação hierárquica com três níveis pode ser utilizado para detectar as possíveis fontes de variação.

Desenvolvemos uma análise Bayesiana assumindo uma densidade a priori nãoinformativa para os parâmetros do modelo $\left(\mu, \sigma_{1}^{2}, \sigma_{2}^{2}, \sigma_{3}^{2}\right)$, também sugerida por Box e Tiao (1973). Para compreendermos o fundamento utilizado por Box e Tiao na obtenção da densidade a priori, é necessário que antes obtenhamos a função de verossimilhança, a qual será apresenta a seguir. 


\subsection{Função de Verossimilhança para o Modelo de Efeitos Aleatórios com 3 Componentes de Variância}

Para obter a função de verossimilhança é mais conveniente trabalhar com as variáveis transformadas $y_{i . .}, y_{i j}-y_{i . .}, y_{i j k}-y_{i j}$. do que com as observações $y_{i j k}$, apenas. Em termos do modelo (1.3) essas quantidades são dadas por

$$
\begin{aligned}
& y_{i . .}=\theta+e_{i}+e_{i .}+e_{i . .}, \\
& y_{i j .}-y_{i . .}=\left(e_{i j .}-e_{i .}\right)+\left(e_{i j .}-e_{i . .}\right) \mathrm{e} \\
& y_{i j k}-y_{i j .}=\left(e_{i j k}-e_{i j .}\right) .
\end{aligned}
$$

Mantendo as suposições usuais para o modelo e, portanto, admitindo que as variáveis aleatórias $e_{i}$, $e_{i j}$ e $e_{i j k}$ são independentes e normalmente distribuídas, ou seja, $e_{i j k} \sim N\left(0, \sigma_{1}^{2}\right), e_{i j} \sim N\left(0 ; \sigma_{2}^{2}\right)$ e $e_{i} \sim N\left(0 ; \sigma_{3}^{2}\right)$, e de acordo com os resultados de (a) a (e) decorrentes do teorema (1.2) (ver capítulo 1), a função de verossimilhança é dada por

$$
\begin{aligned}
L\left(\mu, \sigma_{1}^{2}, \sigma_{12}^{2}, \sigma_{123}^{2} / y\right) & \propto\left(\sigma_{1}^{2}\right)^{-\frac{v_{1}}{2}}\left(\sigma_{12}^{2}\right)^{-\frac{v_{2}}{2}}\left(\sigma_{123}^{2}\right)^{-\frac{\left(v_{3}+1\right)}{2}} \\
& \times \exp \left\{-\frac{1}{2}\left[\frac{J K \sum_{i=1}^{I}\left(y_{i . .}-\mu\right)^{2}}{\sigma_{123}^{2}}+\frac{v_{2} m_{2}}{\sigma_{12}^{2}}+\frac{v_{1} m_{1}}{\sigma_{1}^{2}}\right]\right\} .
\end{aligned}
$$

Observando que, $J K \sum_{i=1}^{I}\left(y_{i . .}-\mu\right)^{2}=I J K\left(y_{\ldots . .}-\mu\right)^{2}+J K \sum_{i=1}^{I}\left(y_{i . .}-y_{\ldots . .}\right)^{2}$, em termos de $\left(\mu, \sigma_{1}^{2}, \sigma_{2}^{2}, \sigma_{3}^{2}\right)$ a função de verossimilhança (4.2) pode ser escrita na forma: 


$$
\begin{aligned}
& L\left(\mu, \sigma_{1}^{2}, \sigma_{2}^{2}, \sigma_{3}^{2} / y\right) \propto\left(\sigma_{1}^{2}\right)^{-\frac{v_{1}}{2}}\left(\sigma_{1}^{2}+K \sigma_{2}^{2}\right)^{-\frac{v_{2}}{2}}\left(\sigma_{1}^{2}+K \sigma_{2}^{2}+J K \sigma_{3}^{2}\right)^{-\frac{\left(v_{3}+1\right)}{2}} \times \\
& \quad \times \exp \left\{-\frac{1}{2}\left[\frac{I J K(y-\mu))^{2}}{\sigma_{1}^{2}+K \sigma_{2}^{2}+J K \sigma_{3}^{2}}+\frac{v_{3} m_{3}}{\sigma_{1}^{2}+K \sigma_{2}^{2}+J K \sigma_{3}^{3}}+\frac{v_{2} m_{2}}{\sigma_{1}^{2}+K \sigma_{2}^{2}}+\frac{v_{1} m_{1}}{\sigma_{1}^{2}}\right]\right\},
\end{aligned}
$$

onde $\left(m_{1}, m_{2}, m_{3}\right)$ e $\left(v_{1}, v_{2}, v_{3}\right)$ são os quadrados médios e graus de liberdades definidos na tabela (1.2).

\subsection{Densidade a Priori Não-Informativa para $\mu, \sigma_{1}^{2}, \sigma_{2}^{2}$ e $\sigma_{3}^{2}$}

Analogamente ao que foi feito para o modelo (1.8), para obter a densidade a priori não-informativa, Box e Tiao (1973) baseiam-se no fato que a função de verossimilhança (4.2) pode ver vista como sendo constituída por $I$ observações independentes com distribuição $N\left(\mu ; \sigma_{123}^{2} / J K\right)$, juntamente com $I(J-1)$ observações independentes com distribuição $N\left(0 ; \sigma_{12}^{2} / K\right)$, e adicionais $I J(K-1)$ observações independentes com distribuição $N\left(0 ; \sigma_{1}^{2}\right)$. Tratando o parâmetro de locação $\mu$, separadamente dos componentes de variância, a densidade a priori para $\left(\mu, \sigma_{1}^{2}, \sigma_{12}^{2}, \sigma_{123}^{2}\right)$ é obtida a partir de distribuição com $\left(\mu, \ln \sigma_{1}^{2}, \ln \sigma_{12}^{2}, \ln \sigma_{123}^{2}\right)$ localmente uniforme e localmente independente. Ou seja, assumindo independência entre os parâmetros $\mu$ e $\left(\sigma_{1}^{2}, \sigma_{12}^{2}, \sigma_{123}^{2}\right)$ a densidade a priori conjunta é dada por

$$
\pi\left(\mu, \sigma_{1}^{2}, \sigma_{12}^{2}, \sigma_{123}^{2}\right)=\pi(\mu) \pi\left(\sigma_{1}^{2}, \sigma_{12}^{2}, \sigma_{123}^{2}\right),
$$

com

$$
\begin{aligned}
& \pi(\mu) \propto \text { Constante, } \\
& \pi\left(\sigma_{1}^{2}, \sigma_{12}^{2}, \sigma_{123}^{2}\right) \propto \sigma_{1}^{-2} \sigma_{12}^{-2} \sigma_{123}^{-2} .
\end{aligned}
$$


Conseqüentemente, em termos de $\left(\mu, \sigma_{1}^{2}, \sigma_{2}^{2}, \sigma_{3}^{2}\right)$, uma densidade a priori nãoinformativa é dada por

$$
\pi\left(\mu, \sigma_{1}^{2}, \sigma_{2}^{2}, \sigma_{3}^{2}\right) \propto \sigma_{1}^{-2}\left(\sigma_{1}^{2}+K \sigma_{2}^{2}\right)^{-1}\left(\sigma_{1}^{2}+K \sigma_{2}^{2}+J K \sigma_{3}^{2}\right)^{-1}
$$

\subsection{Densidade a Posteriori Conjunta para $\mu, \sigma_{1}^{2}, \sigma_{2}^{2}$ e $\sigma_{3}^{2}$}

Combinando a função de verossimilhança (4.3) com a densidade a priori (4.7), obtemos a densidade a posteriori conjunta para $\left(\mu, \sigma_{1}^{2}, \sigma_{2}^{2}, \sigma_{3}^{2}\right)$ (ver por exemplo, Press, 1989) dada por

$$
\begin{aligned}
\pi\left(\mu, \sigma_{1}^{2}, \sigma_{2}^{2}, \sigma_{3}^{2} / y\right) \propto\left(\sigma_{1}^{2}\right)^{-\left(\frac{v_{1}}{2}+1\right)}\left(\sigma_{1}^{2}+K \sigma_{2}^{2}\right)^{-\left(\frac{\nu_{2}}{2}+1\right)}\left(\sigma_{1}^{2}+K \sigma_{2}^{2}+J K \sigma_{3}^{2}\right)^{-\left(\frac{v_{3}}{2}+\frac{3}{2}\right)} \times \\
\quad \times \exp \left\{-\frac{1}{2}\left[\frac{I J K(y,-\mu)^{2}}{\sigma_{1}^{2}+K \sigma_{2}^{2}+J K \sigma_{3}^{2}}+\frac{v_{3} m_{3}}{\sigma_{1}^{2}+K \sigma_{2}^{2}+J K \sigma_{3}^{3}}+\frac{v_{2} m_{2}}{\sigma_{1}^{2}+K \sigma_{2}^{2}}+\frac{v_{1} m_{1}}{\sigma_{1}^{2}}\right]\right\},
\end{aligned}
$$

onde $-\infty<\mu<\infty$ e $\sigma_{1}^{2}, \sigma_{2}^{2}, \sigma_{3}^{2}>0$

\subsubsection{Densidade a Posteriori Marginal Conjunta para $\sigma_{1}^{2}, \sigma_{2}^{2}$ e $\sigma_{3}^{2}$}

Como as inferências sobre o parâmetro $\mu$ não são de nosso interesse, é conveniente elimina-lo da densidade a posteriori conjunta; para tanto, basta integrar (4.8) como relação a $\mu$, obtendo-se assim, uma densidade a posteriori marginal conjunta para $\left(\sigma_{1}^{2}, \sigma_{2}^{2}, \sigma_{3}^{2}\right)$, dada por 


$$
\begin{aligned}
\pi\left(\sigma_{1}^{2}, \sigma_{2}^{2}, \sigma_{3}^{2} / y\right) & \propto\left(\sigma_{1}^{2}\right)^{-\left(\frac{v_{1}}{2}+1\right)}\left(\sigma_{1}^{2}+K \sigma_{2}^{2}\right)^{-\left(\frac{v_{2}}{2}+1\right)}\left(\sigma_{1}^{2}+K \sigma_{2}^{2}+J K \sigma_{3}^{2}\right)^{-\left(\frac{v_{3}}{2}+1\right)} \times \\
& \times \exp \left\{-\frac{1}{2}\left[\frac{v_{3} m_{3}}{\sigma_{1}^{2}+K \sigma_{2}^{2}+J K \sigma_{3}^{3}}+\frac{v_{2} m_{2}}{\sigma_{1}^{2}+K \sigma_{2}^{2}}+\frac{v_{1} m_{1}}{\sigma_{1}^{2}}\right]\right\},
\end{aligned}
$$

onde $\sigma_{1}^{2}, \sigma_{2}^{2}, \sigma_{3}^{2}>0$.

\subsubsection{Densidade a Posteriori Marginal para a Razão $\sigma_{2}^{2} / \sigma_{1}^{2}$}

Também para esse caso, pode ser muito útil conhecer o comportamento da razão de variâncias $\sigma_{2}^{2} / \sigma_{1}^{2}$, principalmente quando o objetivo é alocar as unidades amostrais entre os níveis de amostragem (ver por exemplo, Box, Hunter e Hunter, 1978). Por conveniência matemática trabalhamos com a razão $\sigma_{12}^{2} / \sigma_{1}^{2}$, ao invés da razão $\sigma_{2}^{2} / \sigma_{1}^{2}$. Para obter a densidade a posteriori de interesse, consideramos a seguinte transformação de variáveis:

$$
\begin{aligned}
& V=\sigma_{1}^{2}, \\
& W=\frac{\sigma_{12}^{2}}{\sigma_{1}^{2}}=1+K \frac{\sigma_{2}^{2}}{\sigma_{1}^{2}}, \\
& U=\sigma_{1}^{2}+K \sigma_{2}^{2}+J K \sigma_{3}^{2} .
\end{aligned}
$$

Conseqüentemente, temos $\sigma_{1}^{2}=V, \sigma_{2}^{2}=(W-1) V / K$ e $\sigma_{3}^{2}=(U-W V) / J K$, com o Jacobiano da transformação dado por 


$$
J(V, W, U)=\left|\begin{array}{lll}
\frac{\partial \sigma_{1}^{2}}{\partial V} & \frac{\partial \sigma_{1}^{2}}{\partial W} & \frac{\partial \sigma_{1}^{2}}{\partial U} \\
\frac{\partial \sigma_{2}^{2}}{\partial V} & \frac{\partial \sigma_{2}^{2}}{\partial W} & \frac{\partial \sigma_{2}^{2}}{\partial U} \\
\frac{\partial \sigma_{3}^{2}}{\partial V} & \frac{\partial \sigma_{3}^{2}}{\partial W} & \frac{\partial \sigma_{3}^{2}}{\partial U}
\end{array}\right|=\left|\begin{array}{ccc}
1 & 0 & 0 \\
\frac{W-1}{K} & \frac{V}{K} & 0 \\
\frac{-W}{J K} & \frac{-V}{J K} & \frac{1}{J K}
\end{array}\right|
$$

Assim, $|J(V, W, V)|=V\left(J K^{2}\right)^{-1}$ e portanto, usando a regra de transformação de variáveis e de acordo com a densidade a posteriori marginal conjunta (4.9), a densidade a posteriori conjunta para $(V, W, U)$ é dada por

$$
\pi(w, v, u / y) \propto(w)^{-\left(\frac{v_{2}}{2}+1\right)}(u)^{-\left(\frac{v_{3}}{2}+1\right)}(v)^{-\left(\frac{v_{1}+v_{2}}{2}+1\right)} \exp \left\{-\frac{1}{2}\left[\frac{v_{3} m_{3}}{u}+\frac{v_{2} m_{2}}{w v}+\frac{v_{1} m_{1}}{v}\right]\right\},
$$

onde $w>1, v, u>0$.

Para obter a densidade a posteriori marginal para W, basta integrar (4.11) com relação a $\mathrm{V}$ e U, e para isso utilizamos o resultado (3.1) (capítulo 3) de onde obtemos,

$$
\pi(w / y) \propto(w)^{-\left(\frac{v_{2}}{2}+1\right)}\left[\frac{v_{2} m_{2}}{w}+v_{1} m_{1}\right]^{-\frac{\left(v_{1}+v_{2}\right)}{2}}
$$

onde $w>1$.

Derivando o logaritmo de (4.12) em relação a $w$, igualando a zero e resolvendo a equação em $w$, encontramos a moda dessa densidade a posteriori marginal, a qual é dada por

$$
\tilde{w}=\frac{v_{2} m_{2}\left(v_{1}-2\right)}{v_{1} m_{1}\left(v_{2}+2\right)} .
$$




\subsubsection{Densidade a Posteriori para Outras Funções de $\sigma_{1}^{2}, \sigma_{2}^{2}$ e $\sigma_{3}^{2}$}

Outras funções dos parâmetros podem ser de grande interesse para o pesquisador, como por exemplo, a razão de variâncias $\sigma_{123}^{2} / \sigma_{1}^{2}$. Considerando a nova transformação de variáveis dada por

$$
\begin{aligned}
& V=\sigma_{1}^{2}, \\
& W=\frac{\sigma_{12}^{2}}{\sigma_{1}^{2}}=1+K \frac{\sigma_{2}^{2}}{\sigma_{1}^{2}}, \\
& T=\frac{\sigma_{123}^{2}}{\sigma_{1}^{2}}=1+K \frac{\sigma_{2}^{2}}{\sigma_{1}^{2}}+J K \frac{\sigma_{3}^{2}}{\sigma_{1}^{2}} .
\end{aligned}
$$

Conseqüentemente, temos $\sigma_{1}^{2}=V, \sigma_{2}^{2}=V(W-1) / K$ e $\sigma_{3}^{2}=V(T-W) / J K$ e o Jacobiano da transformação dado por

$$
J(V, W, T)=\left|\begin{array}{lll}
1 & 0 & 0 \\
\frac{W-1}{K} & \frac{V}{K} & 0 \\
\frac{(T-W)}{J K} & \frac{-V}{J K} & \frac{V}{J K}
\end{array}\right| . \text { Portanto }|J(V, W, T)|=V^{2}\left(J K^{2}\right)^{-1}
$$

Assim, a densidade a posteriori conjunta para V, W e T é dada por

$$
\pi(v, w, t / y) \propto(v)^{-\left(\frac{v_{1}+v_{2}+v_{3}}{2}+1\right)}(w)^{-\left(\frac{v_{2}}{2}+1\right)}(t)^{-\left(\frac{v_{3}}{2}+1\right)} \exp \left\{-\frac{1}{2 v}\left[\frac{v_{3} m_{3}}{t}+\frac{v_{2} m_{2}}{w}+v_{1} m_{1}\right]\right\},
$$

onde $v>0, w, t>1$. 
Para calcular as densidades a posteriori marginais para $\mathrm{V}, \mathrm{W}$ e $\mathrm{T}$, recorremos ao fato de que, para um conjunto de dados que não apresente problemas de estimativas negativas de $\sigma_{2}^{2}$ e $\sigma_{3}^{2}$, valores de $\mathrm{W}$ e $\mathrm{T}$ tais que, $0<w, t<1$ assumem densidade zero. Desse modo, a densidade a posteriori marginal para $\mathrm{V}$ pode ser expressa na forma:

$$
\pi(v / y) \propto v^{-\left(\frac{v_{1}+v_{2}+v_{3}}{2}+1\right)} e^{-\frac{v_{1} m_{1}}{2 v}} \cdot \int_{0}^{\infty} \int_{0}^{\infty} w^{-\left(\frac{v_{2}}{2}+1\right)} t^{-\left(\frac{v_{3}}{2}+1\right)} \exp \left\{-\frac{1}{2 v}\left[\frac{v_{3} m_{3}}{t}+\frac{v_{2} m_{2}}{w}\right]\right\} d w d t
$$

Aplicando sucessivamente o resultado (3.1), obtemos a seguinte expressão para a posteriori marginal para V:

$$
\pi(v / y) \propto v^{-\left(\frac{v_{1}}{2}+1\right)} e^{-\frac{v_{1} m_{1}}{2 v}},
$$

onde $v>0$. Podemos observar que a densidade em (4.16) é uma densidade Gama Invertida, cuja moda a posteriori é dada por

$$
\tilde{v}=\frac{v_{1} m_{1}}{\left(v_{1}+2\right)}
$$

A densidade a posteriori para $\mathrm{W}$ é obtida integrando-se (4.15) com relação a $\mathrm{V}$ e T, ou seja,

$$
\pi(w / y) \propto w^{-\left(\frac{v_{2}}{2}+1\right)} \int_{0}^{\infty} \int_{0}^{\infty} v^{-\left(\frac{v_{1}+\nu_{2}+\nu_{3}}{2}+1\right)} t^{-\left(\frac{v_{3}}{2}+1\right)} \exp \left\{-\frac{1}{2 v}\left[\frac{v_{3} m_{3}}{t}+\frac{v_{2} m_{2}}{w}+v_{1} m_{1}\right]\right\} d v d t .
$$


Resolvendo primeiramente a integral em $\mathrm{V}$, pois em $\mathrm{V}$ a integral pode ser resolvida analiticamente, a densidade a posteriori para $\mathrm{W}$ pode ser escrita na seguinte forma:

$$
\pi(w / y) \propto w^{-\left(\frac{\nu_{2}}{2}+1\right)} \int_{0}^{\infty} e^{-n h_{w}(t)} d t,
$$

onde,

$$
-n h_{w}(t)=-\left(\frac{v_{3}}{2}+1\right) \ln (t)-\left(\frac{v_{1}+v_{2}+v_{3}}{2}\right) \ln \left[\frac{v_{3} m_{3}}{t}+\frac{v_{2} m_{2}}{w}+v_{1} m_{1}\right]
$$

Utilizando o método de Laplace para resolver a integral em (4.18) (ver capítulo 2), temos que a densidade a posteriori marginal aproximada para $\mathrm{W}$ é dada por

$$
\pi(w / y) \propto w^{-\left(\frac{v_{2}}{2}+1\right)}\left[\frac{v_{2} m_{2}}{w}+v_{1} m_{1}\right]^{-\left(\frac{v_{1}+v_{2}}{2}\right)},
$$

onde $w>1$.

Podemos observar que essa densidade aproximada coincide com a densidade a posteriori marginal para $\mathrm{W}$ em (4.12), obtida através de resultados exatos.

Para obter a densidade a posteriori marginal para $\mathrm{T}$ integramos primeiramente em V, utilizando o resultado (3.1), e então obtemos a expressão,

$$
\pi(t / y) \propto t^{-\left(\frac{v_{3}}{2}+1\right)} \int e^{-n h_{t}(w)} d w
$$

$$
\text { onde, }-n h_{t}(w)=-\left(\frac{v_{2}}{2}+1\right) \ln (w)-\left(\frac{v_{1}+v_{2}+v_{3}}{2}\right) \ln \left[\frac{v_{3} m_{3}}{t}+\frac{v_{2} m_{2}}{w}+v_{1} m_{1}\right] \text {. }
$$


Uma aproximação de Laplace para a integral em (4.20) resulta na seguinte aproximação para a densidade a posteriori marginal para $\mathrm{T}$ :

$$
\pi(t / y) \propto t^{-\left(\frac{v_{3}}{2}+1\right)}\left[\frac{v_{3} m_{3}}{t}+v_{1} m_{1}\right]^{-\left(\frac{v_{1}+v_{3}}{2}\right)},
$$

onde $t>1$, e a moda da densidade é dada por

$$
\tilde{t}=\frac{v_{3} m_{3}\left(v_{1}-2\right)}{v_{1} m_{1}\left(v_{3}+2\right)} .
$$

Também utilizamos o método de Laplace para obter momentos a posteriori de algumas funções dos componentes de variância, mais especificamente, das variáveis $\mathrm{V}$, W e T definidas em (4.14), para os quais obtemos os resultados:

$$
\begin{gathered}
\hat{E}(v / y) \approx \frac{e\left(v_{1} m_{1}\right)\left(\frac{v_{1}}{2}\right)^{\frac{\left(v_{1}-3\right)}{2}}}{2\left(\frac{v_{1}}{2}+1\right)^{\frac{\left(v_{1}-1\right)}{2}}}, \\
\hat{E}(w / y) \approx \frac{\left(v_{2} m_{2}\right)\left(\frac{v_{1}}{2}+2\right)^{\frac{\left(v_{1}+1\right)}{2}}\left(\frac{v_{2}}{2}\right)^{\frac{\left(v_{2}-3\right)}{2}}}{\left(v_{1} m_{1}\right)\left(\frac{v_{1}}{2}+1\right)^{\frac{\left(v_{1}-1\right)}{2}}\left(\frac{v_{2}}{2}+1\right)^{\frac{\left(v_{2}-1\right)}{2}}}, \\
\hat{E}(t / y) \approx \frac{\left(v_{3} m_{3}\right)\left(\frac{v_{1}}{2}+2\right)^{\frac{\left(v_{1}+1\right)}{2}}\left(\frac{v_{3}}{2}\right)^{\frac{\left(v_{3}-3\right)}{2}}}{\left(v_{1} m_{1}\right)\left(\frac{v_{1}}{2}+1\right)^{\frac{\left(v_{1}-1\right)}{2}}\left(\frac{v_{3}}{2}+1\right)^{\frac{\left(v_{3}-1\right)}{2}} .}
\end{gathered}
$$


Podemos observar que as expressões para a média a posteriori aproximada de $\sigma_{1}^{2}\left(\sigma_{1}^{2}=V\right)$ e de $\sigma_{12}^{2} / \sigma_{1}^{2}\left(\sigma_{12}^{2} / \sigma_{1}^{2}=W\right)$ coincidem com as obtidas para o modelo com 2 componentes de variância (ver capítulo 3 ) quando consideramos a parametrização original e a densidade a priori (3.6) (observar expressões (3.28a) e (3.29b)).

Devemos lembrar novamente, que ao utilizarmos um método de aproximação devemos estar cientes da precisão dos resultados obtidos. Como já foi dito no capítulo 3, o método de aproximação de Laplace proporciona resultados mais precisos quando trabalhamos com uma densidade a priori localmente uniforme (ver por exemplo, Achcar e Smith, 1990). No entanto, podemos observar que a densidade a priori considerada (4.7) não é localmente uniforme (ver por exemplo, Box e Tiao, 1973),o que pode implicar em pouca precisão nas aproximações para os momentos a posteriori.

\subsection{Reparametrização Logarítmica em $\sigma_{1}^{2}, \sigma_{12}^{2}$ e $\sigma_{123}^{2}$}

Como vimos na seção (4.2), a densidade a priori (4.7) é obtida a partir de uma distribuição com $\left(\mu, \ln \sigma_{1}^{2}, \ln \sigma_{12}^{2}, \ln \sigma_{123}^{2}\right)$ localmente uniforme e localmente independente. Assim se assumimos a reparametrização $\mu=\mu$, $\xi_{1}=\ln \left(\sigma_{1}^{2}\right), \xi_{2}=K^{-1} \ln \left(\sigma_{12}^{2}\right), \quad \xi_{3}=(J K)^{-1} \ln \left(\sigma_{123}^{2}\right)$, a densidade a priori para $\left(\mu, \xi_{1}, \xi_{2}, \xi_{3}\right)$ de acordo com (4.7) é dada por

$$
\pi\left(\mu, \xi_{1}, \xi_{2}, \xi_{3}\right) \propto \text { Constante. }
$$

Portanto, na parametrização $\left(\mu, \xi_{1}, \xi_{2}, \xi_{3}\right)$, esperamos obter aproximações mais precisas para os momentos a posteriori considerados na seção anterior.

A função de verosimilhança na nova parametrização e de acordo com (4.3) é dada por 


$$
\begin{aligned}
L\left(\mu, \xi_{1}, \xi_{2}, \xi_{3} / y\right) & \propto\left(e^{\xi_{1}}\right)^{-\frac{\nu_{1}}{2}}\left(e^{K \xi_{2}}\right)^{-\frac{\nu_{2}}{2}}\left(e^{J K \xi_{3}}\right)^{-\frac{\left(\nu_{3}+1\right)}{2}} \times \\
& \times \exp \left\{-\frac{1}{2}\left[\frac{I J K\left(y_{\ldots}-\mu\right)^{2}}{e^{J K \xi_{3}}}+\frac{v_{3} m_{3}}{e^{J K \xi_{3}}}+\frac{v_{2} m_{2}}{e^{K \xi_{2}}}+\frac{v_{1} m_{1}}{e^{\xi_{1}}}\right]\right\} .
\end{aligned}
$$

Combinando a função de verossimilhança (4.27) com a densidade a priori (4.26) obtemos a densidade a posteriori conjunta para $\mu, \xi_{1}, \xi_{2}$ e $\xi_{3}$, dada por

$$
\begin{aligned}
\pi\left(\mu, \xi_{1}, \xi_{2}, \xi_{3} / y\right) & \propto\left(e^{\xi_{1}}\right)^{-\frac{v_{1}}{2}}\left(e^{K \xi_{2}}\right)^{-\frac{v_{2}}{2}}\left(e^{J K \xi_{3}}\right)^{-\frac{\left(\nu_{3}+1\right)}{2}} \times \\
& \times \exp \left\{-\frac{1}{2}\left[\frac{I J K\left(y_{y}-\mu\right)^{2}}{e^{J K \xi_{3}}}+\frac{v_{3} m_{3}}{e^{J K \xi_{3}}}+\frac{v_{2} m_{2}}{e^{K \xi_{2}}}+\frac{v_{1} m_{1}}{e^{\xi_{1}}}\right]\right\},
\end{aligned}
$$

onde $-\infty<\mu, \xi_{1}, \xi_{2}, \xi_{3}<\infty$.

Para obter a densidade a posteriori marginal para os parâmetros $\xi_{1}, \xi_{2}$ e $\xi_{3}$ basta integrar (4.28) em relação a $\mu$. Fazendo isso, obtemos o seguinte resultado:

$$
\pi\left(\xi_{1}, \xi_{2}, \xi_{3} / y\right) \propto\left(e^{\xi_{1}}\right)^{-\frac{\nu_{1}}{2}}\left(e^{K \xi_{2}}\right)^{-\frac{v_{2}}{2}}\left(e^{J K \xi_{3}}\right)^{-\frac{\nu_{3}}{2}} \exp \left\{-\frac{1}{2}\left[\frac{v_{3} m_{3}}{e^{J K \xi_{3}}}+\frac{v_{2} m_{2}}{e^{K \xi_{2}}}+\frac{v_{1} m_{1}}{e^{\xi_{1}}}\right]\right\},
$$

onde $-\infty<\xi_{1}, \xi_{2}, \xi_{3}<\infty$.

Na nova parametrização, as médias a posteriori de $\mathrm{V}, \mathrm{W}$ e $\mathrm{T}$ aproximadas por Laplace são dadas por: 


$$
\begin{gathered}
\hat{E}(v / y) \approx \frac{e\left(v_{1} m_{1}\right)\left(\frac{v_{1}}{2}-1\right)^{\left(\frac{v_{1}-3}{2}\right)}}{2\left(\frac{v_{1}}{2}\right)^{\left(\frac{v_{1}-1}{2}\right)}}, \\
\hat{E}(w / y) \approx \frac{v_{2} m_{2}\left(\frac{v_{1}}{2}+1\right)^{\frac{\left(v_{1}+1\right)}{2}}\left(\frac{v_{2}}{2}-1\right)^{\frac{\left(v_{2}-3\right)}{2}}}{v_{1} m_{1}\left(\frac{v_{1}}{2}\right)^{\frac{\left(v_{1}-1\right)}{2}}\left(\frac{v_{2}}{2}\right)^{\frac{v_{2}-1}{2}}}, \\
\hat{E}(t / y) \approx \frac{\left(v_{3} m_{3}\right)\left(\frac{v_{1}}{2}+1\right)^{\frac{\left(v_{1}+1\right)}{2}}\left(\frac{v_{3}}{2}-1\right)^{\frac{\left(v_{3}-3\right)}{2}}}{\left(v_{1} m_{1}\right)\left(\frac{v_{1}}{2}\right)^{\frac{\left(v_{1}-1\right)}{2}}\left(\frac{v_{3}}{2}\right)^{\frac{\left(v_{3}-1\right)}{2}} .}
\end{gathered}
$$

Novamente observamos que as aproximações para a média a posteriori de $\sigma_{1}^{2}$ $\left(\sigma_{1}^{2}=V\right)$ e de $\sigma_{12}^{2} / \sigma_{1}^{2}\left(\sigma_{12}^{2} / \sigma_{1}^{2}=W\right)$, na parametrização $\left(\xi_{1}, \xi_{2}, \xi_{3}\right)$, coincidem com as encontradas para o modelo com 2 componentes de variância também na parametrização $\left(\xi_{1}, \xi_{2}\right)$ (observar expressões (3.44a) e (3.44c)). 


\section{Capítulo 5}

\section{Análise Bayesiana para o Modelo de Efeitos Aleatórios Não - Normal}

Nesse capítulo, exploramos o uso de métodos Bayesianos aproximados para o modelo de efeitos aleatórios (1.8) assumindo uma distribuição normal $N\left(0, \sigma_{1}^{2}\right)$ para o erro aleatório $e_{j k}$ e uma distribuição mistura de normais com densidade (1.30) para o efeito aleatório $e_{j}$ (ver capítulo 1), baseados no método de Laplace para aproximação de integrais.

O uso de métodos iterativos para resolução de sistemas de equações não-lineares (como por exemplo, Newton Raphson) e o uso de métodos de aproximação de integrais, tornam-se imprescindíveis para esse tipo de problema, devido ao grande número de parâmetros do modelo e a grande complexidade das fórmulas envolvidas. Nesse sentido, uma das grandes vantagens na utilização do método de Laplace para resolver integrais Bayesianas de interesse está em termos do baixo custo computacional e na sua facilidade na implementação, a qual não requer nenhuma experiência computacional sofisticada. 


\subsection{Densidade a Posteriori Conjunta para $\sigma_{1}^{2}, \sigma_{2}^{2}, \phi$ e $\lambda$}

Considerando o modelo de efeitos aleatórios (1.8), assumindo $\mu=0$, e de acordo com os resultados do teorema (1.2) (ver capítulo 1) temos (ver Tiao e Ali, 1971),

a) $y_{j}$. e os resíduos $y_{j k}-y_{j}$. são independentes,

b) a soma de quadrados $S_{1}$ tem distribuição $\sigma_{1}^{2} \chi_{v_{1}}^{2}$,

c) $y_{j}$. e $S_{1}$ são conjuntamente suficientes para $\left(\sigma_{1}^{2}, \sigma_{2}^{2}, \lambda, \phi\right)$,

d) os grupos de médias $y_{j}$. são independentes e identicamente distribuídos com uma distribuição mistura de normais dada por

$$
p\left(y_{j .} / \sigma_{1}^{2}, \sigma_{2}^{2}, \phi, \lambda\right)=A_{1 j}+A_{2 j} \text {, }
$$

onde

$$
\begin{gathered}
y_{j .}=K^{-1} \sum_{k} y_{j k}, v_{1}=J(K-1), v_{1} m_{1}=S_{1}, \\
S_{1}=\sum_{j} \sum_{k}\left(y_{j k}-y_{j .}\right)^{2}, \\
A_{1 j}=\left(0,95 \exp \left\{-\frac{\left[y_{j .}+0,05 \phi(\lambda-1) \sigma\right]^{2}}{2\left(\sigma^{2}+\sigma_{1}^{2} / K\right)}\right\}\right) /\left(\sigma^{2}+\sigma_{1}^{2} / K\right)^{1 / 2}, \\
A_{2 j}=\left(0,05 \exp \left\{-\frac{\left[y_{j .}-0,95 \phi(\lambda-1) \sigma\right]^{2}}{2\left(\lambda^{2} \sigma^{2}+\sigma_{1}^{2} / K\right)}\right\}\right) /\left(\lambda^{2} \sigma^{2}+\sigma_{1}^{2} / K\right)^{1 / 2}, \\
\sigma_{2}^{2}=c(\lambda, \phi) \sigma^{2}, \text { e } c(\lambda, \phi)=0,95+0,05 \lambda^{2}+0,0475 \phi^{2}(\lambda-1)^{2} .
\end{gathered}
$$


Assim, a função de verossimilhança para $\sigma_{1}^{2}, \sigma_{2}^{2}, \phi$ e $\lambda$ é dada por

$$
\begin{aligned}
L\left(\sigma_{1}^{2}, \sigma_{2}^{2}, \phi, \lambda / y\right) & \propto\left(\sigma_{1}^{2}\right)^{-v_{1} / 2} \exp \left\{-\frac{v_{1} m_{1}}{2 \sigma_{1}^{2}}\right\} \times \\
& \times \prod_{j=1}^{J} p\left(y_{j .} / \sigma_{1}^{2}, \sigma_{2}^{2}, \phi, \lambda\right),
\end{aligned}
$$

onde $y$ é um vetor de dados.

A densidade a priori conjunta para $\sigma_{1}^{2}, \sigma_{2}^{2}, \phi$ e $\lambda$ pode ser escrita na forma:

$$
\pi\left(\sigma_{1}^{2}, \sigma_{2}^{2}, \phi, \lambda\right)=\pi\left(\sigma_{1}^{2}, \sigma_{2}^{2} / \phi, \lambda\right) \pi_{0}(\phi, \lambda)
$$

onde $\pi_{0}(\phi, \lambda)$ é uma densidade a priori conjunta para $\phi$ e $\lambda$ e $\pi\left(\sigma_{1}^{2}, \sigma_{2}^{2} / \phi, \lambda\right)$ é uma densidade a priori não-informativa para $\sigma_{1}^{2}$ e $\sigma_{2}^{2}$ dado $\phi$ e $\lambda$. Considerando a priori não-informativa de Box e Tiao (3.6) para $\sigma_{1}^{2}$ e $\sigma_{2}^{2}$ dado $\phi$ e $\lambda$, ou seja, $\pi\left(\sigma_{1}^{2}, \sigma_{2}^{2} / \phi, \lambda\right) \propto\left(\sigma_{1}^{2}\right)^{-1}\left(\sigma_{1}^{2}+K \sigma_{2}^{2}\right)^{-1}$ (também considerada por Tiao e Ali, 1971), a densidade a posteriori conjunta para $\sigma_{1}^{2}, \sigma_{2}^{2}, \phi$ e $\lambda$ é dada por

$$
\pi\left(\sigma_{1}^{2}, \sigma_{2}^{2}, \phi, \lambda / y\right) \propto \pi_{0}(\phi, \lambda)\left(\sigma_{1}^{2}\right)^{-1}\left(\sigma_{1}^{2}+K \sigma_{2}^{2}\right)^{-1} L\left(\sigma_{1}^{2}, \sigma_{2}^{2}, \phi, \lambda / y\right),
$$

onde $\sigma_{1}^{2}>0, \sigma_{2}^{2}>0, \lambda \geq 1, \phi=-1,0,1$; e $L\left(\sigma_{1}^{2}, \sigma_{2}^{2}, \phi, \lambda / y\right)$ é a função de verossimilhança (5.2). 


\subsection{Densidade a Posteriori Marginal Aproximada para $\phi$}

A densidade a posteriori marginal para $\phi($ de (5.4)) é dada por

$$
\pi(\phi / y) \propto \iiint \pi_{0}(\phi, \lambda)\left(\sigma_{1}^{2}\right)^{-1}\left(\sigma_{1}^{2}+K \sigma_{2}^{2}\right)^{-1} L\left(\sigma_{1}^{2}, \sigma_{2}^{2}, \phi, \lambda / y\right) d \sigma_{1}^{2} d \sigma_{2}^{2} d \lambda .
$$

Considerando,

$$
f_{\phi}\left(\sigma_{1}^{2}, \sigma_{2}^{2}, \lambda\right)=\pi_{0}(\phi, \lambda)\left(\sigma_{1}^{2}\right)^{-1}\left(\sigma_{1}^{2}+K \sigma_{2}^{2}\right)^{-1}
$$

$\mathrm{e}$

$$
-n h_{\phi}\left(\sigma_{1}^{2}, \sigma_{2}^{2}, \lambda\right)=\ln L\left(\sigma_{1}^{2}, \sigma_{2}^{2}, \phi, \lambda / y\right)
$$

em (2.3) (ver capítulo 2), uma aproximação de Laplace para a densidade a posteriori marginal para $\phi$ é dada por

$$
\pi(\phi / y) \propto \frac{\pi_{0}(\phi, \hat{\lambda})\left(\hat{\sigma}_{1}^{2}\right)^{-1}\left(\hat{\sigma}_{1}^{2}+K \hat{\sigma}_{2}^{2}\right)^{-1} L\left(\hat{\sigma}_{1}^{2}, \hat{\sigma}_{2}^{2}, \phi, \hat{\lambda} / y\right)}{\left\{\operatorname{det}\left(n \sum_{h_{\phi}}^{-1}\left(\hat{\sigma}_{1}^{2}, \hat{\sigma}_{2}^{2}, \hat{\lambda}\right)\right)\right\}^{1 / 2}}
$$

onde $\phi=-1,0,1$ e $\left(\hat{\sigma}_{1}^{2}, \hat{\sigma}_{2}^{2}, \hat{\lambda}\right)$ maximizam $-n h_{\phi}\left(\sigma_{1}^{2}, \sigma_{2}^{2}, \lambda\right)$ para cada valor de $\phi$.

Uma vez que temos dificuldade para calcular as derivadas de segunda ordem de $-n h\left(\sigma_{1}^{2}, \sigma_{2}^{2}, \lambda\right)$, podemos considerar o uso de derivadas numéricas localmente em $\left(\hat{\sigma}_{1}^{2}, \hat{\sigma}_{2}^{2}, \hat{\lambda}\right)$ (ver apêndice A) para obter um valor aproximado do determinante da matriz Hessiana em (5.6). 
Observe também que a aproximação em (5.6) é válida para qualquer escolha da densidade a priori para os parâmetros $\sigma_{1}^{2}, \sigma_{2}^{2}, \phi$ e $\lambda$.

\subsection{Densidade a Posteriori Marginal Aproximada para $\lambda$ com $\phi$ Conhecido}

A densidade a posteriori marginal para $\lambda$ é dada (de (5.4)) por

$$
\pi(\lambda / y)=\sum_{\phi=-1,0,1} \iint \pi\left(\sigma_{1}^{2}, \sigma_{2}^{2}, \phi, \lambda / y\right) d \sigma_{1}^{2} d \sigma_{2}^{2}
$$

Assumindo $\phi$ conhecido, consideramos a densidade a priori

$$
\begin{aligned}
\pi\left(\sigma_{1}^{2}, \sigma_{2}^{2}, \lambda / \phi\right)= & \pi\left(\sigma_{1}^{2}, \sigma_{2}^{2} / \phi, \lambda\right) \pi_{0}(\lambda / \phi) \\
& \propto\left(\sigma_{1}^{2}\right)^{-1}\left(\sigma_{1}^{2}+K \sigma_{2}^{2}\right)^{-1} \pi_{0}(\lambda / \phi),
\end{aligned}
$$

onde $\pi_{0}(\lambda / \phi)$ é uma densidade a priori para $\lambda$ dado $\phi$.

Com essa priori, a densidade a posteriori conjunta para $\sigma_{1}^{2}, \sigma_{2}^{2}$ e $\lambda$ é dada por

$$
\begin{aligned}
\pi\left(\sigma_{1}^{2}, \sigma_{2}^{2}, \lambda / \phi, y\right) & \propto \pi_{0}(\lambda / \phi)\left(\sigma_{1}^{2}\right)^{-1}\left(\sigma_{1}^{2}+K \sigma_{2}^{2}\right)^{-1} \times \\
& \times L\left(\sigma_{1}^{2}, \sigma_{2}^{2}, \lambda / \phi, y\right) .
\end{aligned}
$$

Com a escolha $f_{\lambda}\left(\sigma_{1}^{2}, \sigma_{2}^{2}\right)=\pi_{0}(\lambda / \phi)\left(\sigma_{1}^{2}\right)^{-1}\left(\sigma_{1}^{2}+K \sigma_{2}^{2}\right)^{-1}$ e $-n h_{\lambda}\left(\sigma_{1}^{2}, \sigma_{2}^{2}\right)=$ $\ln L\left(\sigma_{1}^{2}, \sigma_{2}^{2}, \lambda / \phi, y\right)$ em (2.3), uma aproximação de Laplace para a densidade a posteriori marginal para $\lambda$ é dada por 


$$
\pi(\lambda / \phi, y) \propto \frac{\pi_{0}(\lambda / \phi)\left(\hat{\sigma}_{1}^{2}\right)^{-1}\left(\hat{\sigma}_{1}^{2}+K \hat{\sigma}_{2}^{2}\right)^{-1} L\left(\hat{\sigma}_{1}^{2}, \hat{\sigma}_{2}^{2}, \lambda / \phi, y\right)}{\left\{\operatorname{det}\left(n \sum_{h \lambda}^{-1}\left(\hat{\sigma}_{1}^{2}, \hat{\sigma}_{2}^{2}\right)\right)\right\}^{1 / 2}},
$$

onde $\left(\hat{\sigma}_{1}^{2}, \hat{\sigma}_{2}^{2}\right)$ maximizam $-n h_{\lambda}\left(\sigma_{1}^{2}, \sigma_{2}^{2}\right)$ para cada valor de $\lambda$.

\subsection{Uma Análise Bayesiana com $\phi$ e $\lambda$ Conhecidos}

Assumindo $\phi$ e $\lambda$ conhecidos, a função de verossimilhança para $\sigma_{1}^{2}, \sigma_{2}^{2}$ é (ver (5.2)) dada por

$$
L\left(\sigma_{1}^{2}, \sigma_{2}^{2} / \phi, \lambda, y\right) \propto\left(\sigma_{1}^{2}\right)^{-v_{1} / 2} \exp \left\{-\frac{v_{1} m_{1}}{2 \sigma_{1}^{2}}\right\} \prod_{j=1}^{J}\left\{A_{1 j}+A_{2 j}\right\},
$$

onde,

$$
\begin{aligned}
& A_{1 j}=\left(0,95 \exp \left\{-\frac{\left(y_{j .}+a_{1} \sigma\right)^{2}}{2\left(\sigma^{2}+\sigma_{1}^{2} / K\right)}\right\}\right) /\left(\sigma^{2}+\sigma_{1}^{2} / K\right)^{1 / 2}, \\
& A_{2 j}=\left(0,05 \exp \left\{-\frac{\left(y_{j .}-a_{2} \sigma\right)^{2}}{2\left(\lambda^{2} \sigma^{2}+\sigma_{1}^{2} / K\right)}\right\}\right) /\left(\lambda^{2} \sigma^{2}+\sigma_{1}^{2} / K\right)^{1 / 2}, \\
& a_{1}=0,05 \phi(\lambda-1), a_{2}=0,95 \phi(\lambda-1), \sigma^{2}=\sigma_{2}^{2} / b \mathrm{e} \\
& b=0,95+0,05 \lambda^{2}+0,0475 \phi^{2}(\lambda-1)^{2} .
\end{aligned}
$$


A densidade a posteriori conjunta para $\sigma_{1}^{2}, \sigma_{2}^{2}$ é dada por

$$
\pi\left(\sigma_{1}^{2}, \sigma_{2}^{2} / \phi, \lambda, y\right) \propto \pi\left(\sigma_{1}^{2}, \sigma_{2}^{2} / \phi, \lambda\right) L\left(\sigma_{1}^{2}, \sigma_{2}^{2} / \phi, \lambda, y\right),
$$

onde $\sigma_{1}^{2}>0, \sigma_{2}^{2}>0 ; \pi\left(\sigma_{1}^{2}, \sigma_{2}^{2} / \phi, \lambda\right)$ é uma densidade a priori para $\left(\sigma_{1}^{2}, \sigma_{2}^{2}\right)$ com $\phi$ e $\lambda$ conhecidos e $L\left(\sigma_{1}^{2}, \sigma_{2}^{2} / \phi, \lambda, y\right)$ é a função de verossimilhança (5.11).

\subsubsection{Densidade a Posteriori Marginal Aproximada para $\sigma_{1}^{2}$}

A densidade a posteriori marginal para $\sigma_{1}^{2}$ pode ser escrita (de (5.12)) na forma:

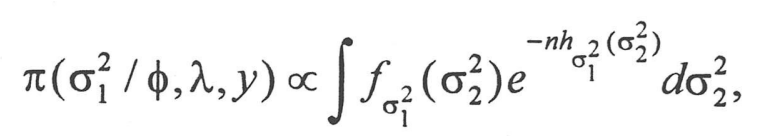

onde $f_{\sigma_{1}^{2}}\left(\sigma_{2}^{2}\right)=\pi\left(\sigma_{1}^{2}, \sigma_{2}^{2} / \phi, \lambda\right)$ ( uma densidade a priori para $\sigma_{1}^{2}$ e $\sigma_{2}^{2}$ dado $\phi$ e $\lambda$ ) e $-n h_{\sigma_{1}^{2}}\left(\sigma_{2}^{2}\right)=\ln L\left(\sigma_{1}^{2}, \sigma_{2}^{2} / \phi, \lambda, y\right)$.

Portanto, uma densidade a posteriori marginal aproximada para $\sigma_{1}^{2}$ usando o método de Laplace é dada por

$$
\pi\left(\sigma_{1}^{2} / \phi, \lambda, y\right) \propto \frac{\pi\left(\sigma_{1}^{2}, \hat{\sigma}_{2}^{2} / \phi, \lambda\right) L\left(\sigma_{1}^{2}, \hat{\sigma}_{2}^{2}, \lambda / \phi, \lambda, y\right)}{\left\{-\frac{\partial^{2} g\left(\sigma_{1}^{2}, \hat{\sigma}_{2}^{2}\right)}{\partial\left(\sigma_{2}^{2}\right)^{2}}\right\}^{1 / 2}},
$$

onde $\sigma_{1}^{2}>0, \hat{\sigma}_{2}^{2}$ maximiza $-n h_{\sigma_{1}^{2}}\left(\sigma_{2}^{2}\right)$ para cada valor de $\sigma_{1}^{2}$ e $g\left(\sigma_{1}^{2}, \sigma_{2}^{2}\right)=$ $\sum_{j=1}^{J} \ln \left(A_{1 j}+A_{2 j}\right)$. 


\subsubsection{Densidade a Posteriori Marginal Aproximada para $\sigma_{2}^{2}$}

A densidade a posteriori marginal para $\sigma_{2}^{2}$ pode ser escrita (de (5.12)) na forma:

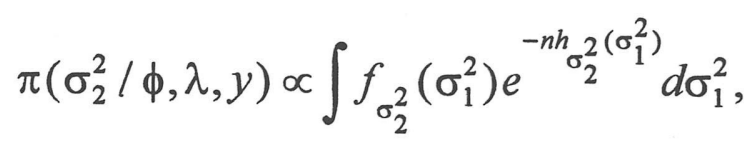

onde $f_{\sigma_{2}^{2}}\left(\sigma_{1}^{2}\right)=\pi\left(\sigma_{1}^{2}, \sigma_{2}^{2} / \phi, \lambda\right)$ e $-n h_{\sigma_{2}^{2}}\left(\sigma_{1}^{2}\right)=\ln L\left(\sigma_{1}^{2}, \sigma_{2}^{2} / \phi, \lambda, y\right)$.

Uma densidade a posteriori marginal aproximada para $\sigma_{2}^{2}$ é dada por

$$
\pi\left(\sigma_{2}^{2} / \phi, \lambda, y\right) \propto \frac{\pi\left(\hat{\sigma}_{1}^{2}, \sigma_{2}^{2} / \phi, \lambda, y\right)}{\left\{\frac{v_{1} m_{1}}{\left(\hat{\sigma}_{1}^{2}\right)^{3}}-\frac{v_{1}}{2\left(\hat{\sigma}_{1}^{2}\right)^{2}}-\frac{\partial^{2} g\left(\hat{\sigma}_{1}^{2}, \sigma_{2}^{2}\right)}{\partial\left(\sigma_{1}^{2}\right)^{2}}\right\}^{1 / 2}},
$$

onde $\sigma_{2}^{2}>0, \hat{\sigma}_{1}^{2}$ maximiza $-n h_{\sigma_{2}^{2}}\left(\sigma_{1}^{2}\right)$ para cada valor de $\sigma_{2}^{2}$.

\subsubsection{Distribuição Preditiva Aproximada para uma Média de um Grupo Futuro $Y_{(J+1)}$.}

Assumindo $\phi$ e $\lambda$ conhecidos, a densidade preditiva para uma média de um grupo futuro $y_{(J+1)}$. é dada por

$$
p\left(y_{(J+1)} / y\right) \propto \iint p\left(y_{(J+1)} / \sigma_{1}^{2}, \sigma_{2}^{2}, \phi, \lambda\right) \pi\left(\sigma_{1}^{2}, \sigma_{2}^{2} / \phi, \lambda, y\right) d \sigma_{1}^{2} d \sigma_{2}^{2},
$$


onde $p\left(y_{(J+1)} \cdot / \sigma_{1}^{2}, \sigma_{2}^{2}, \phi, \lambda\right)=A_{1(J+1)}+A_{2(J+1)}$ e $A_{i j}$ é definido em (5.11).

Utilizando $o$ método de Laplace com $f\left(\sigma_{1}^{2}, \sigma_{2}^{2}\right)=\pi\left(\sigma_{1}^{2}, \sigma_{2}^{2} / \phi, \lambda\right)$ e $-n h\left(\sigma_{1}^{2}, \sigma_{2}^{2}\right)=\ln p\left(y_{(J+1)} / \sigma_{1}^{2}, \sigma_{2}^{2}, \phi, \lambda\right)+\ln L\left(\sigma_{1}^{2}, \sigma_{2}^{2}, \phi, \lambda / y\right)$ em (2.3), obtemos uma densidade preditiva aproximada para uma média de um grupo futuro $y_{(J+1)}$. dada por

$$
p\left(y_{(J+1)} / y\right) \propto \frac{p\left(y_{(J+1)} \cdot / \hat{\sigma}_{1}^{2}, \hat{\sigma}_{2}^{2}, \phi, \lambda\right) \pi\left(\hat{\sigma}_{1}^{2}, \hat{\sigma}_{2}^{2} / \phi, \lambda\right) L\left(\hat{\sigma}_{1}^{2}, \hat{\sigma}_{2}^{2} / \phi, \lambda, y\right)}{\left\{\operatorname{det}\left(n \sum_{h}^{-1}\left(\hat{\sigma}_{1}^{2}, \hat{\sigma}_{2}^{2}\right)\right)\right\}^{1 / 2}},
$$

onde $-\infty<y_{(J+1)} .<\infty ; \hat{\sigma}_{1}^{2}$ e $\hat{\sigma}_{2}^{2}$ maximizam $-n h\left(\sigma_{1}^{2}, \sigma_{2}^{2}\right)$ para cada valor de $y_{(J+1)}$.

\subsection{Uma Análise Bayesiana Assumindo $\lambda=1$ e $\mu=0$}

Assumindo $\lambda=1$ na densidade mistura de normais (1.32) (observar capítulo 1 ), ou seja, uma distribuição normal para o efeito aleatório $e_{j}$, com $\mu=0$ no modelo de efeitos aleatórios (1.8), a função de verosimilhança para $\sigma_{1}^{2}$ e $\sigma_{2}^{2}$, é dada por

$$
L\left(\sigma_{1}^{2}, \sigma_{2}^{2} / y\right) \propto\left(\sigma_{1}^{2}\right)^{-v_{1} / 2} \exp \left\{-\frac{v_{1} m_{1}}{2 \sigma_{1}^{2}}\right\}\left(\sigma_{1}^{2}+K \sigma_{1}^{2}\right)^{-J / 2} \exp \left\{-\frac{K \sum y_{j}^{2}}{2\left(\sigma_{1}^{2}+K \sigma_{2}^{2}\right)}\right\} .
$$

Considerando $f$ iguais à densidade a priori $\pi\left(\sigma_{1}^{2}, \sigma_{2}^{2}\right)$ em $(2.3)$, obtemos as densidades a posteriori marginais aproximadas para $\sigma_{1}^{2}$ e $\sigma_{2}^{2}$ dadas por

$$
\pi\left(\sigma_{1}^{2} / y\right) \propto \frac{\pi\left(\sigma_{1}^{2}, \hat{\sigma}_{2}^{2} / y\right) L\left(\sigma_{1}^{2}, \hat{\sigma}_{2}^{2} / y\right)}{\left\{\frac{K^{3} \sum y_{j}^{2}}{\left(\sigma_{1}^{2}+K \hat{\sigma}_{2}^{2}\right)^{3}}-\frac{J K^{2}}{2\left(\sigma_{1}^{2}+K \hat{\sigma}_{2}^{2}\right)^{2}}\right\}^{1 / 2}},
$$


onde $\sigma_{1}^{2}>0$ e $\hat{\sigma}_{2}^{2}$ maximiza $L\left(\sigma_{1}^{2}, \sigma_{2}^{2} / y\right)$ para cada valor fixo de $\sigma_{1}^{2}$, e

$$
\pi\left(\sigma_{2}^{2} / y\right) \propto \frac{\pi\left(\hat{\sigma}_{1}^{2}, \sigma_{2}^{2} / y\right) L\left(\hat{\sigma}_{1}^{2}, \sigma_{2}^{2} / y\right)}{\left\{\frac{v_{1} m_{1}}{\left(\hat{\sigma}_{1}^{2}\right)^{3}}-\frac{v_{1}}{2\left(\hat{\sigma}_{1}^{2}\right)^{2}}-\frac{J}{2\left(\hat{\sigma}_{1}^{2}+K \sigma_{2}^{2}\right)^{2}}+\frac{K \sum y_{j}^{2}}{2\left(\hat{\sigma}_{1}^{2}+K \sigma_{2}^{2}\right)^{3}}\right\}^{1 / 2}},
$$

onde $\sigma_{2}^{2}>0$ e $\hat{\sigma}_{1}^{2}$ maximiza $L\left(\sigma_{1}^{2}, \sigma_{2}^{2} / y\right)$ para cada valor fixo de $\sigma_{2}^{2}$.

\subsection{Densidade a Posteriori para $\theta$}

Uma maneira de analisarmos o comportamento do parâmetro $\theta$ no modelo com mistura de normais é considerando-o como uma parâmetro desconhecido. Assumindo $\phi$ e $\lambda$ conhecidos, a função de verossimilhança pode ser escrita (de 5.2) na forma:

$$
\begin{aligned}
L\left(\sigma_{1}^{2}, \sigma_{2}^{2}, \theta / \phi, \lambda, y\right) \propto\left(\sigma_{1}^{2}\right)^{-v_{1} / 2} \exp \left\{-\frac{v_{1} m_{1}}{2 \sigma_{1}^{2}}\right\} \times \\
\times \prod_{j=1}^{J} p\left(y_{j} / \sigma_{1}^{2}, \sigma_{2}^{2}, \theta, \phi, \lambda\right),
\end{aligned}
$$

onde,

$$
\begin{gathered}
p\left(y_{j .} / \sigma_{1}^{2}, \sigma_{2}^{2}, \theta, \phi, \lambda\right)=A_{1 j}+A_{2 j}, \\
A_{1 j}=\left((1-\theta) \exp \left\{-\frac{\left[y_{j .}+\theta \phi(\lambda-1) \sigma\right]^{2}}{2\left(\sigma^{2}+\sigma_{1}^{2} / K\right)}\right\}\right) /\left(\sigma^{2}+\sigma_{1}^{2} / K\right)^{1 / 2}, \\
A_{2 j}=\left(\theta \exp \left\{-\frac{\left[y_{j .}-(1-\theta) \phi(\lambda-1) \sigma\right]^{2}}{2\left(\lambda^{2} \sigma^{2}+\sigma_{1}^{2} / K\right)}\right\}\right) /\left(\lambda^{2} \sigma^{2}+\sigma_{1}^{2} / K\right)^{1 / 2} \mathrm{e} \\
\sigma_{2}^{2}=c(\theta) \sigma^{2}, \mathrm{e} c(\theta)=(1-\theta)+\theta \lambda^{2}+\theta(1-\theta) \phi^{2}(\lambda-1)^{2} .
\end{gathered}
$$


Uma densidade a priori para os parâmetros $\left(\sigma_{1}^{2}, \sigma_{2}^{2}, \theta\right)$ pode ser escrita na forma:

$$
\pi\left(\sigma_{1}^{2}, \sigma_{2}^{2}, \theta / \phi, \lambda\right)=\pi\left(\sigma_{1}^{2}, \sigma_{2}^{2} / \theta, \phi, \lambda\right) \pi_{0}(\theta / \phi, \lambda),
$$

onde $\pi_{0}(\theta / \phi, \lambda)$ é uma densidade a priori para $\theta$ assumindo $\phi$ e $\lambda$ conhecidos e $\pi\left(\sigma_{1}^{2}, \sigma_{2}^{2} / \theta, \phi, \lambda\right)$ é uma densidade a priori para $\left(\sigma_{1}^{2}, \sigma_{2}^{2}\right)$ dado $\theta, \phi$ e $\lambda$ Vamos assumir a mesma densidade a priori para os parâmetros $\left(\sigma_{1}^{2}, \sigma_{2}^{2}\right)$ utilizada até aqui, ou seja, $\pi\left(\sigma_{1}^{2}, \sigma_{2}^{2} / \theta, \phi, \lambda\right) \propto\left(\sigma_{1}^{2}\right)^{-1}\left(\sigma_{1}^{2}+K \sigma_{2}^{2}\right)^{-1}$.

Para a escolha da densidade a priori $\pi_{0}(\theta / \phi, \lambda)$ devemos levar em conta que $\theta$ representa probabilidade do processo estar fora de controle e portanto é razoável admitir que algum conhecimento sobre este parâmetro seja disponível por um especialista no início do estudo. Assim, uma densidade a priori que incorpora conhecimento prévio é dada pela distribuição Beta, $B(a, b)$, ou seja,

$$
\pi_{0}(\theta / \phi, \lambda) \propto \theta^{a-1}(1-\theta)^{b-1},
$$

onde $0 \leq \theta \leq 1$ e $a, b>0$. A escolha de $(a, b)$, pode ser feita com base em informações prévias sobre a média e a variância de $\theta$.

Combinando a função de verossimilhança (5.22) com a densidade a priori (5.23) obtemos uma densidade a posteriori conjunta para $\left(\sigma_{1}^{2}, \sigma_{2}^{2}, \theta\right)$ dada por

$$
\pi\left(\sigma_{1}^{2}, \sigma_{2}^{2}, \theta / \phi, \lambda, y\right) \propto \pi\left(\sigma_{1}^{2}, \sigma_{2}^{2}, \theta / \phi, \lambda\right) L\left(\sigma_{1}^{2}, \sigma_{2}^{2}, \theta / \phi, \lambda, y\right) .
$$

A densidade a posteriori marginal para $\theta$ é dada na forma:

$$
\pi(\theta / \phi, \lambda, y) \propto \iint \pi\left(\sigma_{1}^{2}, \sigma_{2}^{2}, \theta / \phi, \lambda\right) L\left(\sigma_{1}^{2}, \sigma_{2}^{2}, \theta / \phi, \lambda, y\right) d \sigma_{1}^{2} d \sigma_{2}^{2} .
$$


Considerando $f_{\theta}\left(\sigma_{1}^{2}, \sigma_{2}^{2}\right)=\pi\left(\sigma_{1}^{2}, \sigma_{2}^{2}, \theta / \phi, \lambda\right)$ (a densidade a priori) e $-n h_{\theta}\left(\sigma_{1}^{2}, \sigma_{2}^{2}\right)=\ln L\left(\sigma_{1}^{2}, \sigma_{2}^{2}, \theta / \phi, \lambda, y\right)$ em (2.3), uma densidade a posteriori aproximada para $\theta$ é dada por

$$
\pi(\theta / \phi, \lambda, y) \propto \frac{\pi\left(\hat{\sigma}_{1}^{2}, \hat{\sigma}_{2}^{2}, \theta / \phi, \lambda\right) L\left(\hat{\sigma}_{1}^{2}, \hat{\sigma}_{2}^{2}, \theta / \phi, \lambda, y\right)}{\left\{\operatorname{det}\left(n \sum_{h_{\theta}}^{-1}\left(\hat{\sigma}_{1}^{2}, \hat{\sigma}_{2}^{2}\right)\right)\right\}^{1 / 2}}
$$

onde $0 \leq \theta \leq 1, \hat{\sigma}_{1}^{2}$ e $\hat{\sigma}_{2}^{2}$ maximizam $-h_{\theta}\left(\sigma_{1}^{2}, \sigma_{2}^{2}\right)$ para cada valor fixo de $\theta$ e $\sum_{h_{\theta}}^{-1}\left(\hat{\sigma}_{1}^{2}, \hat{\sigma}_{2}^{2}\right)$ é a matriz Hessiana de $h_{\theta}\left(\sigma_{1}^{2}, \sigma_{2}^{2}\right)$ calculada em $\hat{\sigma}_{1}^{2}$ e $\hat{\sigma}_{2}^{2}$. 


\section{Capítulo 6}

\section{Exemplos de Aplicação}

Para ilustrar a aplicação das técnicas desenvolvidas nos capítulos anteriores, apresentamos alguns conjuntos de dados, para os quais desenvolvemos uma análise clássica e Bayesiana. Primeiramente consideramos exemplos envolvendo duas fontes de variação e onde é assumido normalidade para os erros e efeitos aleatórios. Posteriormente, analisaremos um conjunto de dados assumindo o modelo com mistura de normais.

\subsection{Exemplo 1}

O primeiro conjunto de dados que analisamos é dado por Davis (1958) e é utilizado também por Box e Tiao (1973). O objetivo do experimento foi investigar se diferentes lotes de uma matéria-prima utilizada na fabricação de uma tinta causava grande ou significante variação na qualidade do produto final. $\mathrm{O}$ experimento consistiu em tomar 6 amostras do material, cada uma representando lotes aleatoriamente selecionados e 5 porções de tinta foram fabricadas a partir de cada amostra. A produção equivalente de cada porção, em gramas de cor padrão, foi determinada por 
um teste analítico e os resultados obtidos estão representados na tabela (6.1), onde a última linha da tabela refere-se a média observada em cada lote.

Seguindo a notação adotada nos capítulos anteriores, nesse exemplo $j=1, \ldots, 6$ refere-se a lotes e $k=1, \ldots, 5$ refere-se a análises. $\mathrm{O}$ componente de variância $\sigma_{1}^{2}$ que associamos a "análise " na verdade compreende os erros devido ao teste analítico e também, possíveis erros amostrais, e o componente $\sigma_{2}^{2}$ que associamos a "lotes" representa a variação associada as diferentes fontes de onde a matéria prima foi selecionada.

Tabela 6.1. Conjunto de dados: exemplo 1 .

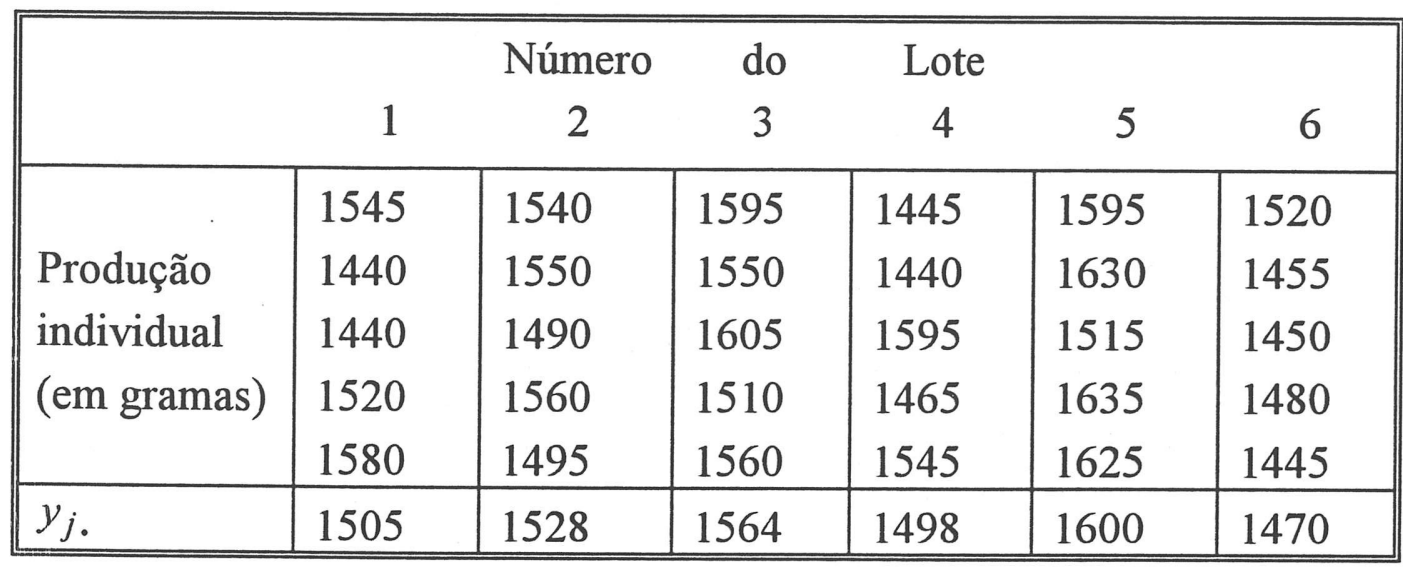

\subsubsection{Análise Clássica do Problema}

Para efetuar uma análise clássica do problema, utilizamos a metodologia desenvolvida no capítulo 1. A tabela (6.2), construída de acordo com a tabela (1.3), representa uma quadro de análise de variância apropriado para o problema. 
Tabela 6.2. Análise de variância para o exemplo 1.

\begin{tabular}{|l|l|l|l||}
\hline Fonte de Var. & S.Q. & G.L. & Q.M. \\
\hline Lotes & $S_{2}=56.357,5$ & $v_{2}=5$ & $m_{2}=11.271,50$ \\
Análises & $S_{1}=58.830,0$ & $v_{1}=24$ & $m_{1}=2.451,25$ \\
Total & $115.187,5$ & 29 & \\
\hline
\end{tabular}

Estimadores não viciados de $\sigma_{1}^{2}$ e $\sigma_{2}^{2}$, obtidos através de análise de variância de acordo com (1.10), são dados por $\hat{\sigma}_{1}^{2}=2451,25$ e $\hat{\sigma}_{2}^{2}=1764,05$.

Considerando a distribuição das somas de quadrados $S_{1}$ e $S_{2}$, intervalos de confiança $95 \%$ para $\sigma_{1}^{2}$ e para a razão $\sigma_{2}^{2} / \sigma_{1}^{2}$ (ver (1.20) e (1.21)) são $(1494,50 ; 4743,90)$ e $(0,09 ; 5,57)$, respectivamente.

De acordo com (1.19) os estimadores de máxima verossimilhança para $\sigma_{1}^{2}$ e $\sigma_{2}^{2}$ são $\hat{\sigma}_{1}^{2}=2451,25$ e $\hat{\sigma}_{2}^{2}=1388,33$. Com base na aproximação Normal assintótica desses estimadores, intervalos de confiança $95 \%$ em (1.29) são dado por $(1064,32 ; 3838,17)$ e $(-755,50 ; 3532,70)$ para $\sigma_{1}^{2}$ e $\sigma_{2}^{2}$ respectivamente. Observamos que esse intervalo de confiança para $\sigma_{2}^{2}$ apresenta problema, pois inclui valores negativos.

\subsubsection{Análise Bayesiana para o Exemplo 1}

Em inferência Bayesiana, as inferências sobre os parâmetros de interesse são obtidas utilizando-se as densidades a posteriori dos mesmos. Portando, considerando as densidades a priori não-informativas (3.3) e (3.6), construímos o gráfico (ver figura (6.1)) da densidade a posteriori marginal para $\sigma_{1}^{2}$ (3.13), obtida pelo método de aproximação de Laplace, lembrando que essa densidade a posteriori marginal é igual para as duas densidades a priori consideradas. A moda dessa densidade a posteriori, de acordo com (3.14), é dada por $\tilde{\sigma}_{1}^{2}=2262,69$.

Similarmente, construímos os gráficos (ver figura (6.2)) das densidades a posteriori marginais para $\sigma_{2}^{2}(3.16)$ e (3.17) obtidas pelo método de aproximação de 
Laplace e considerando as densidades a priori não-informativas (3.3) e (3.6). As modas dessas densidades são dadas por $\tilde{\sigma}_{2}^{2}=908,20$ e $\tilde{\sigma}_{2}^{2}=1104,0$ respectivamente.

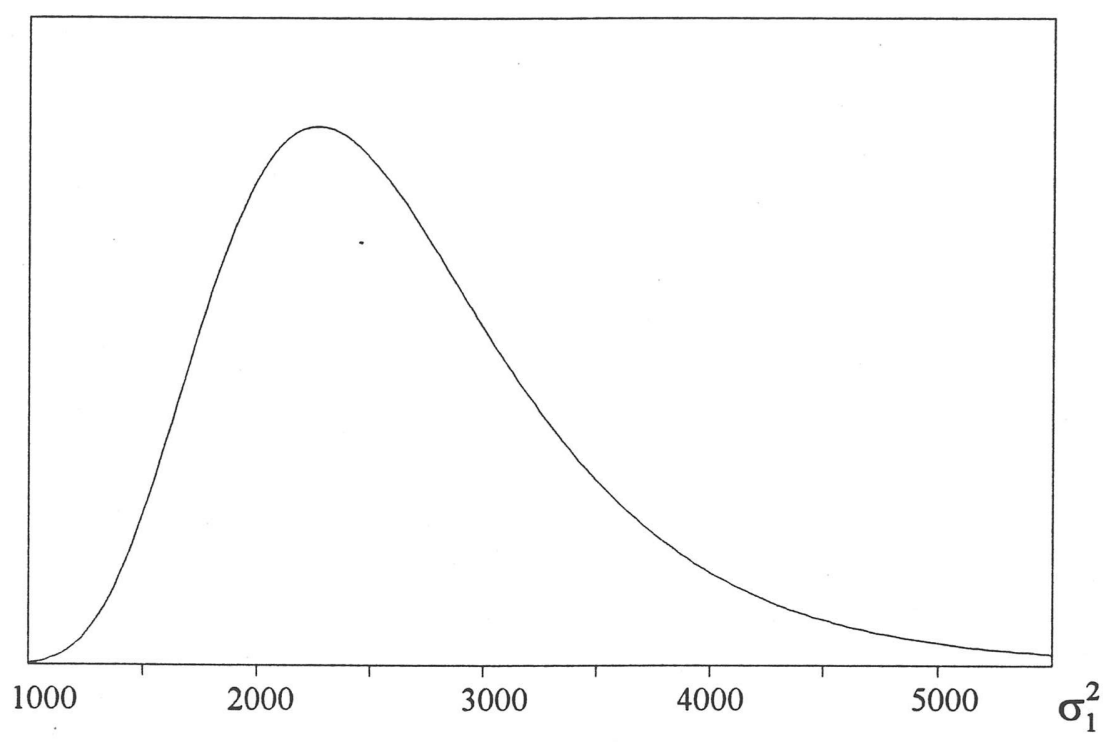

Figura 6.1 - Gráfico da densidade a posteriori marginal para $\sigma_{1}^{2}$.

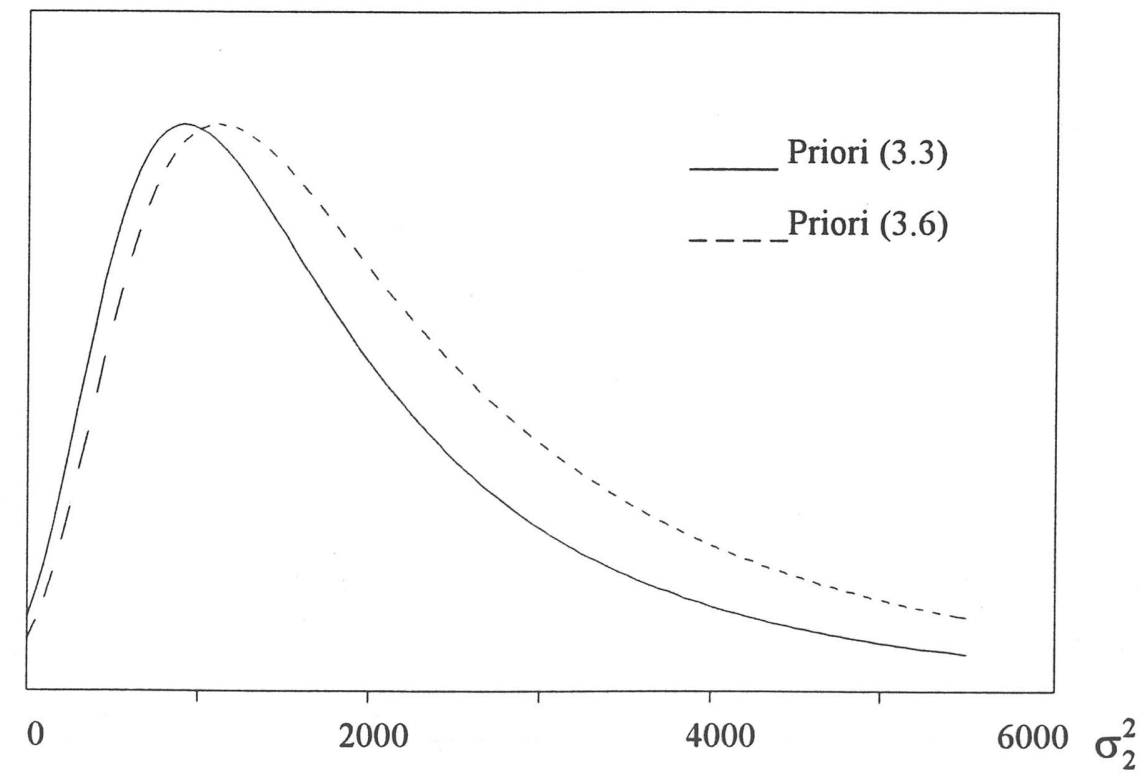

Figura 6.2 - Gráfico das densidades a posteriori marginais para $\sigma_{2}^{2}$. 
Para obter as modas das densidades a posteriori para $\sigma_{2}^{2}$, utilizamos métodos numéricos, através de programas computacionais de fácil operação, executáveis em qualquer microcomputador.

Intervalos de credibilidade aproximados para os parâmetros $\sigma_{1}^{2}$ e $\sigma_{2}^{2}$ são obtidos utilizando-se integração numérica (mais precisamente, a Regra de Simpson ) para calcular as áreas aproximadas abaixo das curvas de suas densidade marginais, ou seja, para obter os pontos $a_{1}, a_{2}$ e $b_{1}, b_{2}$, tais que $P\left(a_{1} \leq \sigma_{1}^{2} \leq a_{2}\right)=0,95$ e $P\left(b_{1} \leq \sigma_{2}^{2} \leq b_{2}\right)=0,95$, nas figuras (6.1) e (6.2) respectivamente. Procedendo dessa maneira, um intervalo de credibilidade a $95 \%$ para $\sigma_{1}^{2}$ é dado por $(1494,5 ; 4743,0)$; similarmente para $\sigma_{2}^{2}$ intervalos de credibilidade a $95 \%$ são dados por $(236,95$; $8463,50)$ e $(325,35 ; 10035,58)$ de acordo com as densidades a priori $(3.3)$ e (36) respectivamente.

Da mesma forma, obtemos intervalos de credibilidade aproximados para a razão de variâncias $\sigma_{2}^{2} / \sigma_{1}^{2}$, a partir das densidades a posteriori marginais para $W\left(W=1+K \sigma_{2}^{2} / \sigma_{1}^{2}\right)$ (ver figura 6.3 ) obtidas de acordo com as densidades a priori (3.3) e (3.6), os quais são dados por $(0,07 ; 3,61)$ e $(0,10 ; 5,21)$ respectivamente.

Um possível valor de previsão para a média de um grupo futuro de observações $y_{21}$. é dado pelas modas das densidades preditivas (3.24) e (3.25). Podemos observar que os resultados são muito semelhantes para qualquer priori considerada (observar figura (6.4)), sendo que a moda é dada por $\tilde{y}_{21}=1527,50$ para ambas densidades preditivas. 


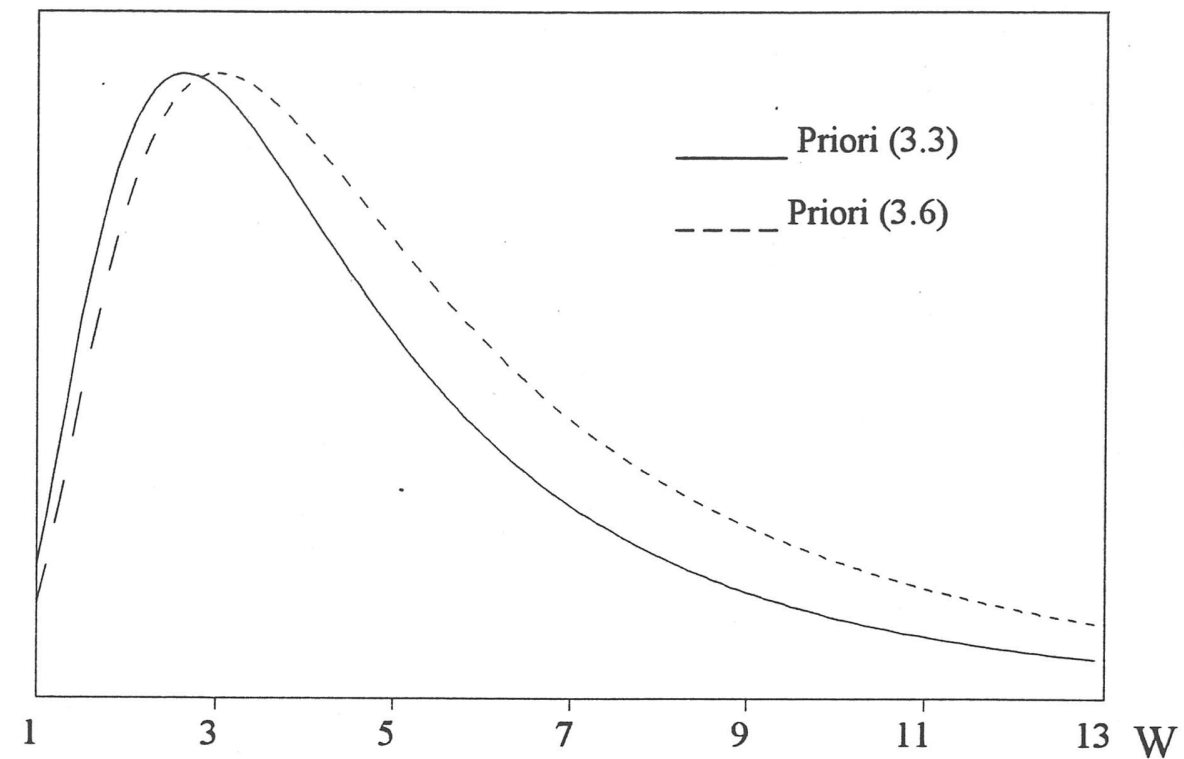

Figura 6.3 - Gráficos das densidades a posteriori marginais para W.

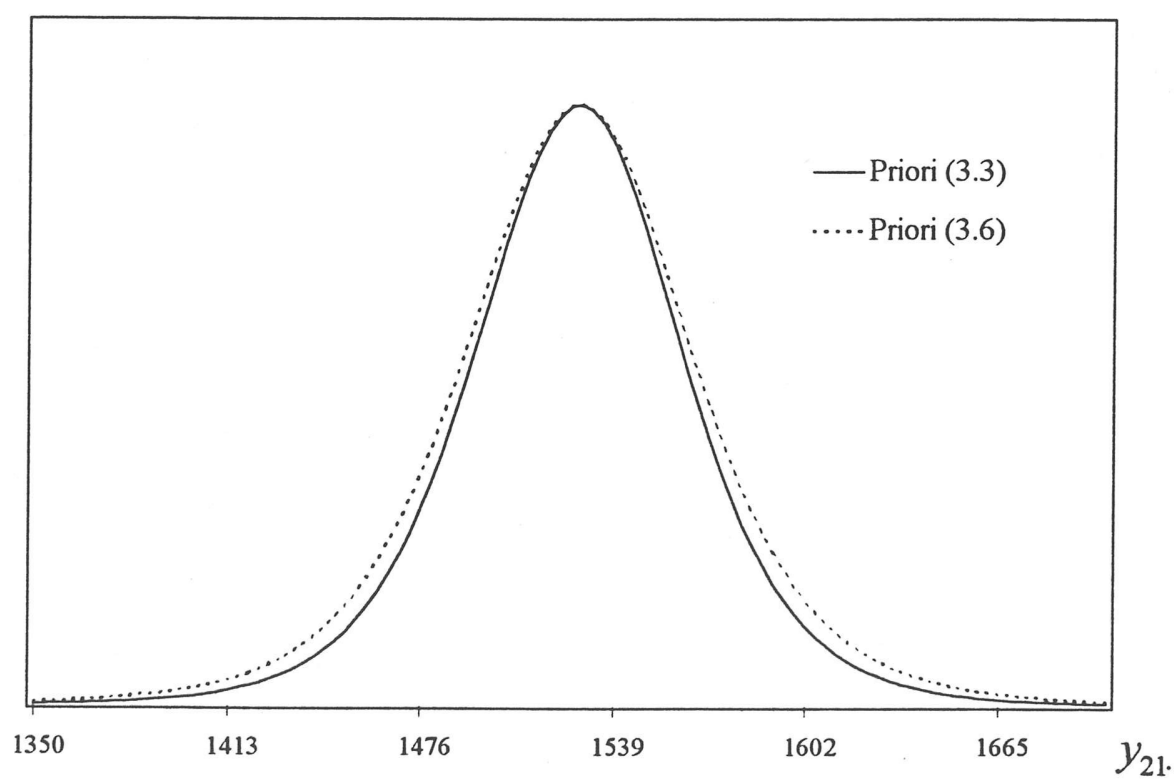

Figura 6.4 - Densidade preditiva para uma média de um grupo futuro. 


\subsubsection{Comparação Entre as Estimativas Obtidas}

$\mathrm{Na}$ tabela (6.3) temos um resumo dos estimadores obtidos via análise de variância, máxima verossimilhança e as modas das densidades a posteriori marginais para $\sigma_{1}^{2}$ e $\sigma_{2}^{2}$.

Tabela 6.3. Estimadores clássicos e modas a posteriori para os parâmetros $\sigma_{1}^{2}$ e $\sigma_{2}^{2}$.

\begin{tabular}{||l|l|l|l||}
\hline Parâmetro & \multicolumn{1}{|c|}{$\begin{array}{c}\text { Estimador de } \\
\text { Análise de } \\
\text { Variância }\end{array}$} & $\begin{array}{c}\text { Estimador de } \\
\text { Máxima Veross. }\end{array}$ & \multicolumn{1}{|c|}{$\begin{array}{c}\text { Moda a } \\
\text { posteriori }\end{array}$} \\
\hline$\sigma_{1}^{2}$ & $\hat{\sigma}_{1}^{2}=2451,25$ & $\hat{\sigma}_{1}^{2}=2451,25$ & $\tilde{\sigma}_{1}^{2}=2262,69$ \\
\hline$\sigma_{2}^{2}$ & $\hat{\sigma}_{2}^{2}=1764,05$ & $\hat{\sigma}_{2}^{2}=1388,33$ & $\begin{array}{l}\tilde{\sigma}_{2}^{2} \approx 908,20 \\
\tilde{\sigma}_{2}^{2} \approx 1104,00\end{array}$ \\
\hline
\end{tabular}

Da tabela (6.3) podemos observar que existe uma divergência entre as estimativas pontuais, principalmente de $\sigma_{2}^{2}$, e não apenas entre os estimadores clássicos e Bayesianos, como também entre os estimadores clássicos de máxima verossimilhança e de análise de variância. Entretanto, devemos lembrar que existe a vantagem do estimador Bayesiano de $\sigma_{2}^{2}$ nunca apresentar valores negativos, uma vez que sua densidade é, de forma natural, restrita a valores maiores que zero e, nesse sentido, a inferência Bayesiana torna-se preferível.

Esse fato pode ser melhor observado na tabela (6.4), onde apresentamos um resumo dos intervalos de confiança e credibilidade para os parâmetros $\sigma_{1}^{2}$ e $\sigma_{2}^{2}$. Podemos observar que o intervalo de credibilidade aproximado para $\sigma_{1}^{2}$ é praticamente igual ao intervalo de confiança obtido através da distribuição de $S_{1}$, o que indica grande eficiência dos métodos de aproximação. $O$ mesmo ocorre com a razão $\sigma_{2}^{2} / \sigma_{1}^{2}$, principalmente se utilizamos a densidade a priori (3.6). Para o parâmetro $\sigma_{2}^{2}$, vemos que os intervalos Bayesianos são mais distintos do clássico, porém não apresentam o problema de incluir valores inadmissíveis para o parâmetro. 
Tabela 6.4. Intervalos de confiança e de credibilidade $95 \%$ para os parâmetros $\sigma_{1}^{2}$ e $\sigma_{2}^{2}$.

\begin{tabular}{||c|l|c|c|c||}
\hline \hline Tipo de intervalo & $\begin{array}{l}\text { Procedi- } \\
\text { mento }\end{array}$ & Parâmetro $\sigma_{1}^{2}$ & Parâmetro $\sigma_{2}^{2}$ & $\begin{array}{c}\text { Razão } \\
\sigma_{2}^{2} / \sigma_{1}^{2}\end{array}$ \\
\hline $\begin{array}{c}\text { Norm. Assintótica } \\
\text { (Int. de confiança) }\end{array}$ & Exato & $(1464,32 ; 3838,17)$ & $(-755,50 ; 3532,70)$ & - \\
\cline { 2 - 5 } $\begin{array}{c}\text { Soma de Quadrado } \\
S_{1} \mathrm{e} S_{2}\end{array}$ & Exato & $(1494,50 ; 4743,90)$ & - & $(0,09 ; 5,57)$ \\
$\begin{array}{c}\text { Intervalo de } \\
\text { Credibilidade }\end{array}$ & $\begin{array}{l}\text { Integração } \\
\text { Numérica }\end{array}$ & $\begin{array}{c}(1494,50 ; 4743,00)^{1} \\
(1494,50 ; 4743,00)^{2}\end{array}$ & $\begin{array}{c}(236,95 ; 8463,50)^{1} \\
(325,35 ; 10035,58)^{2}\end{array}$ & $\begin{array}{c}(0,07 ; 3,61)^{1} \\
(0,10 ; 5,21)^{2}\end{array}$ \\
\hline
\end{tabular}

NOTA: (1) indica que o intervalo de credibilidade foi obtido utilizando-se a densidade a priori (3.3) e (2) a densidade a priori (3.6).

\subsubsection{Verificação da Normalidade das Densidades a Posteriori}

Antes de apresentarmos os resultados obtidos pelo método de aproximação de Laplace, utilizamos técnicas de diagnóstico de normalidade (ver capítulo 3) para avaliarmos a normalidade das densidades a posteriori envolvidas, pois como já foi dito, a precisão dos resultados pode estar comprometida se essas densidades não apresentarem uma boa aproximação normal.
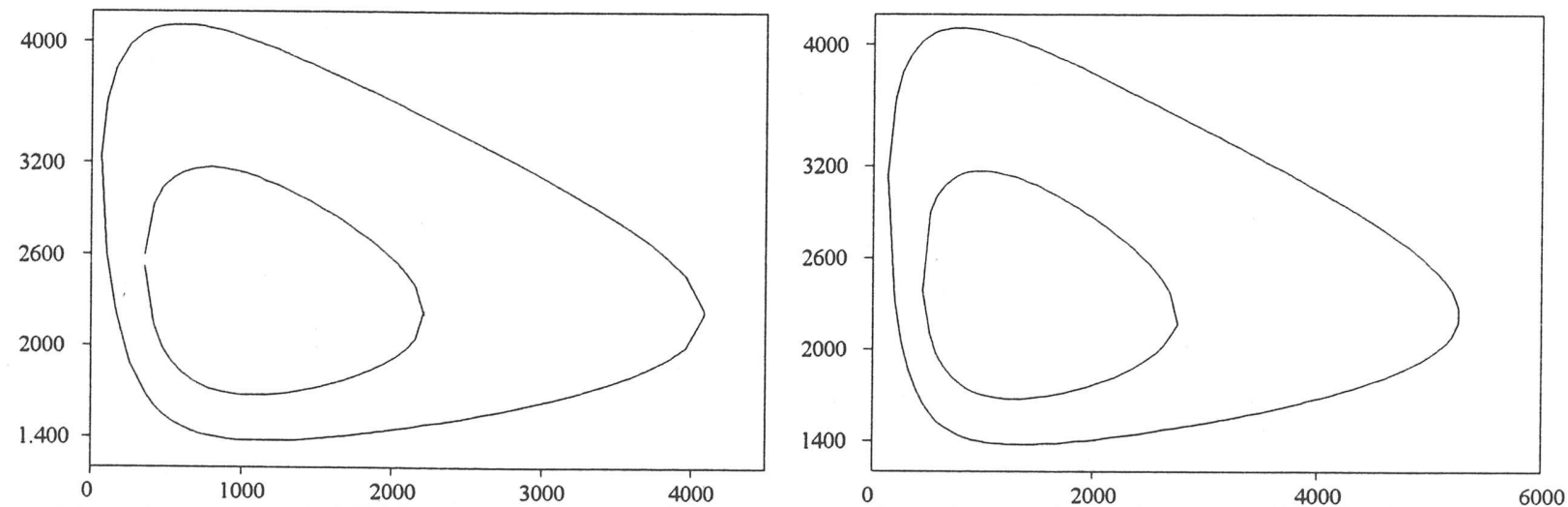

Figura 6.5 - Contornos das densidades posteriori marginais conjuntas para $\left(\sigma_{1}^{2}, \sigma_{2}^{2}\right)$. 


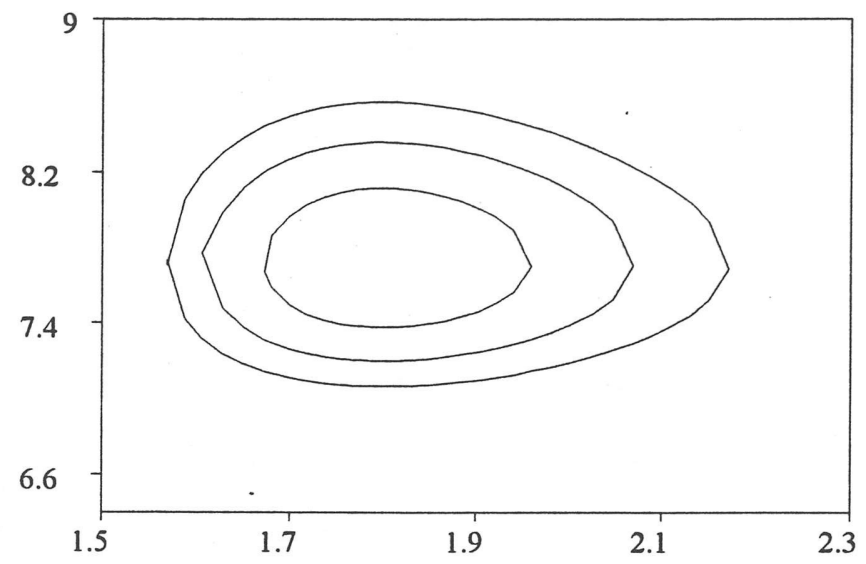

Figura 6.6 - Contornos da densidade posteriori marginal conjunta para $\left(\xi_{1}, \xi_{2}\right)$.

Nesse contexto, na figura (6.5) temos os contornos aproximados (ver Apêndice B sobre a obtenção de contornos de regiões HPD) das densidades a posteriori marginais conjuntas (3.10) e (3.11), respectivamente, para os parâmetros $\sigma_{1}^{2}$ e $\sigma_{2}^{2}$, e observamos que não temos uma boa forma elíptica, enquanto que na figura (6.6), observamos que os contornos aproximados da densidade a posteriori (3.46) para os parâmetros, $\xi_{1}=\ln \left(\sigma_{1}^{2}\right) ; \xi_{2}=K^{-1} \ln \left(\sigma_{1}^{2}+K \sigma_{2}^{2}\right)$ apresentam formas mais próximas de elipses, indicando uma melhor aproximação Normal.

Esse fato é também verificado observando-se as figuras (6.7) e (6.8) onde temos os gráficos T-plot de Hills e Smith para as densidades a posteriori "profile" para os parâmetros $\sigma_{1}^{2}$ e $\sigma_{2}^{2}$, utilizando a densidade a priori (3.3) e (3.6) respectivamente ${ }^{1}$. Podemos observar claramente que essas figuras apresentam formas curvilíneas, indicando a não normalidade dessas densidades a posteriori para os parâmetros $\left(\sigma_{1}^{2}, \sigma_{2}^{2}\right)$.

Porém, considerando a reparametrização $\left(\xi_{1}, \xi_{2}\right)$, o T-plot obtido mostra uma relação aproximadamente linear (ver figura (6.9)), indicando uma melhor aproximação Normal para a densidade a posteriori para esse parâmetros, e portanto esperamos obter aproximações de Laplace com maior precisão nessa parametrização.

\footnotetext{
${ }^{1}$ Maiores detalhes sobre a obtenção do T-plot são apresentados no Apêndice C
} 

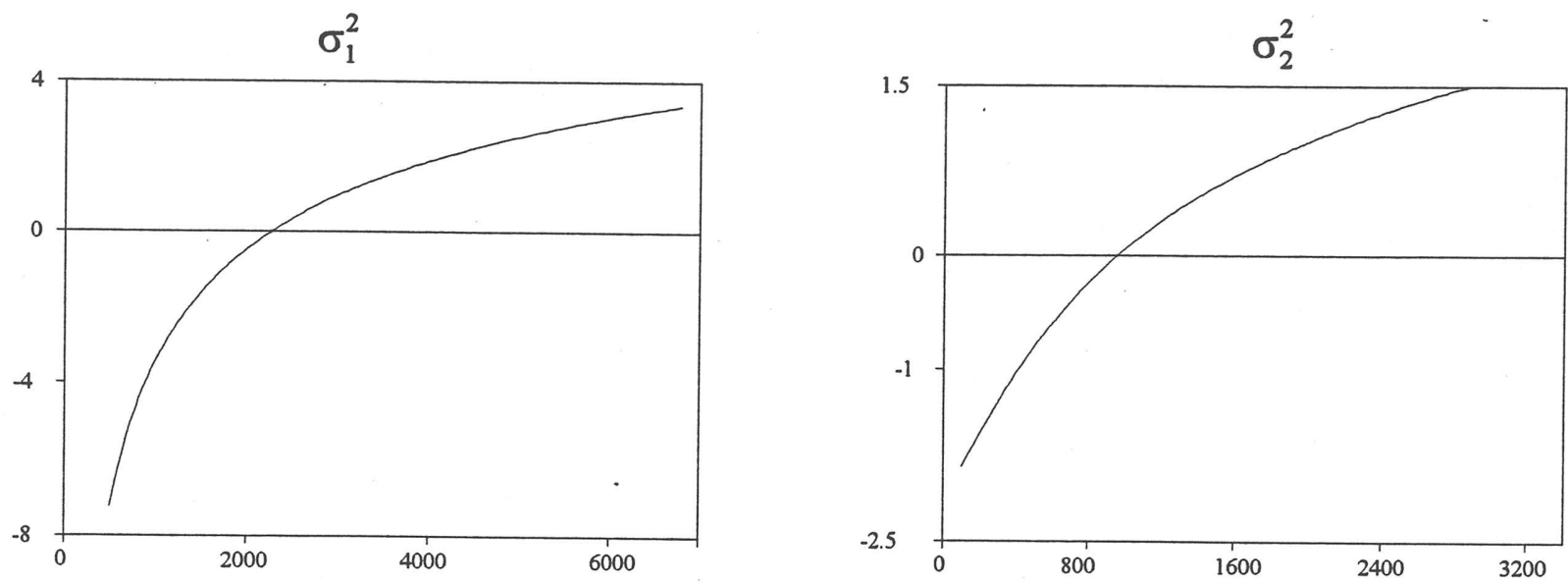

Figura 6.7- T-plot's da posteriori "profile", de acordo com a densidade a priori (3.3).
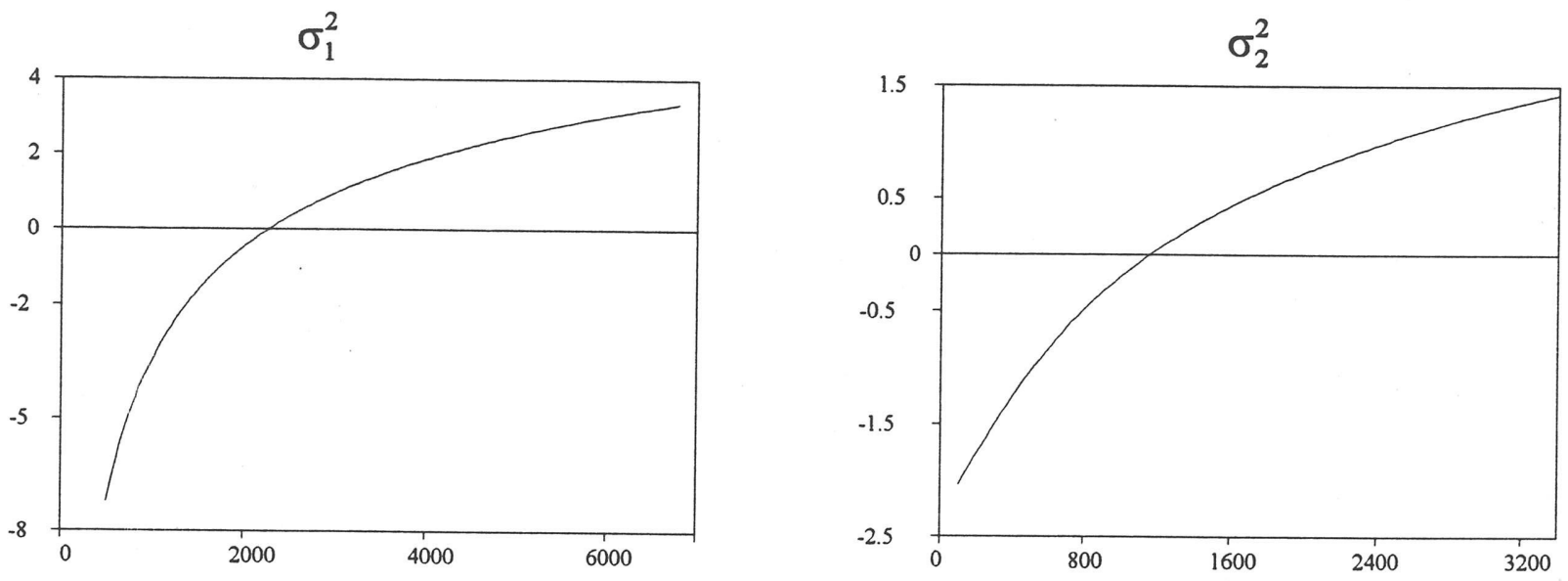

Figura 6.8 - T-plot's da posteriori "profile", de acordo com a densidade a priori (3.6).
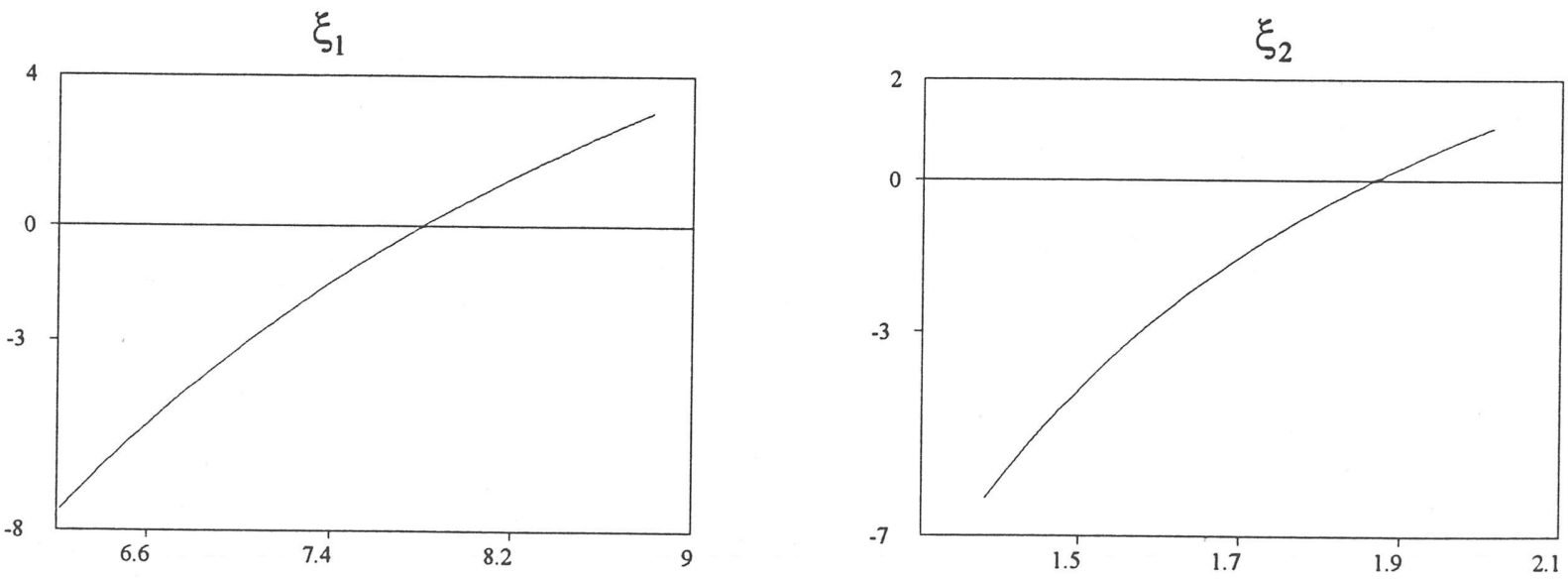

Figura 6.9 - T-plot's da posteriori "profile" considerando a reparametrização. 
A medida de não-normalidade $m^{2} \bar{B}^{2}$ das densidades a posteriori marginais conjuntas para $\left(\sigma_{1}^{2}, \sigma_{2}^{2}\right)$ (ver capítulo 3) obtidas são 2,958 e 3,129, de acordo com as densidade a priori (3.3) e (3.6) respectivamente; na parametrização $\left(\xi_{1}, \xi_{2}\right)$ obtemos o valor 0,483 , indicando novamente melhor normalidade da densidade a posteriori para os parâmetros $\xi_{1}$ e $\xi_{2}$.

\subsubsection{Obtenção de Momentos a Posteriori Exatos e Aproximados}

Considerando as densidades a priori (3.3) e (3.6), calculamos os momentos a posteriori aproximados pelo método de Laplace quando consideramos a parametrização original (ver seção(3.4)) e quando considerando a parametrização com a densidade a priori (3.6) localmente uniforme (ver subseção (3.6.1)). Para comparar a precisão nos resultados aproximados, calculamos também as fórmulas exatas desses momentos a posteriori (ver seção (3.5)). Na tabela (6.5), apresentamos resumidamente esses momentos a posteriori. Abaixo de cada valor aproximado, encontram-se os erros relativos de cada aproximação em relação ao valor exato.

Tabela 6.5. Momentos a posteriori exatos e aproximados para funções $\sigma_{1}^{2}$ e $\sigma_{2}^{2}$.

\begin{tabular}{|c|c|c|c|c|c|}
\hline & $\begin{array}{c}\text { Valores } \\
\text { Priori }(3.3)\end{array}$ & $\begin{array}{l}\text { Exatos } \\
\text { Priori 3.6) }\end{array}$ & $\begin{array}{r}\text { Aprox. de } \\
\left(\sigma_{1}^{2},\right. \\
\text { Priori }(3.3)\end{array}$ & $\begin{array}{l}\text { Laplace na } \\
\left.\sigma_{2}^{2}\right) \\
\text { Priori (3.6) }\end{array}$ & $\begin{array}{l}\text { Parametr. } \\
\left(\xi_{1}, \xi_{2}\right)\end{array}$ \\
\hline$E\left(\sigma_{1}^{2} / y\right)$ & 2658,72 & 2665,00 & $\begin{array}{l}\mathbf{2 6 5 4 , 1 0} \\
(0,17 \%) \\
\end{array}$ & $\begin{array}{l}\mathbf{2 6 5 4 , 1 0} \\
(0,42 \%)\end{array}$ & $\begin{array}{l}\mathbf{2 6 7 2 , 4 0} \\
(0,27 \%)\end{array}$ \\
\hline$E\left(\sigma_{2}^{2} / y\right)$ & 2302,61 & 3237,33 & $\begin{array}{l}\mathbf{2 0 0 2 , 1 0} \\
(15,01 \%)\end{array}$ & $\begin{array}{l}\mathbf{2 6 5 8 , 8 9} \\
(21,75 \%)\end{array}$ & $\begin{array}{l}\mathbf{3 1 6 7 , 4 0} \\
(2,21 \%) \\
\end{array}$ \\
\hline$E\left(\sigma_{12}^{2} / y\right)$ & 14171,75 & 18851,87 & $\begin{array}{l}\mathbf{1 2 4 3 7 , 9 3} \\
(13,94 \%)\end{array}$ & $\begin{array}{l}\mathbf{1 5 6 3 2 , 2 0} \\
(20,60 \%)\end{array}$ & $\begin{array}{l}\mathbf{1 8 3 8 3 , 4 7} \\
(2,55 \%)\end{array}$ \\
\hline$E\left(\frac{\sigma_{12}^{2}}{\sigma_{1}^{2}} / y\right)$ & 5,79 & 7,69 & $\begin{array}{l}\mathbf{5 , 1 1} \\
(13,31 \%)\end{array}$ & $\begin{array}{l}\mathbf{6 , 4 2} \\
(19,78 \%)\end{array}$ & $\begin{array}{l}7,50 \\
(2,53 \%)\end{array}$ \\
\hline
\end{tabular}


Da tabela (6.5) podemos concluir que existe uma influência das densidades a priori consideradas nos momentos a posteriori. Para as duas densidades a priori verificamos que existe um grande erro nas aproximações de Laplace quando obtidas sob a parametrização original $\sigma_{1}^{2}$ e $\sigma_{2}^{2}$ e, como esperávamos, ocorre uma grande redução nesses erros quando utilizamos a parametrização $\xi_{1}$ e $\xi_{2}$ a qual envolve uma densidade a priori localmente uniforme (ver, por exemplo Box e Tiao, 1973). 


\subsection{Exemplo 2}

$\mathrm{Na}$ tabela (6.6) temos um conjunto de dados, o qual foi introduzido por Box e Tiao (1973). Utilizando o modelo (1.8), foram simulados dados de 6 grupos cada um como 5 observações. Os valores utilizados para simulação foram $\mu=5, \sigma_{1}^{2}=16 \mathrm{e}$ $\sigma_{2}^{2}=4$. Para simplificar, continuamos a associar os componentes $\sigma_{1}^{2}$ e $\sigma_{2}^{2}$ a análises e lotes, como no exemplo 1.

Tabela 6.6. Conjunto de dados simulados.

\begin{tabular}{||c|c|c|c|c|c|c||}
\hline \hline & \multicolumn{7}{|c}{\begin{tabular}{c} 
Número \\
\hline
\end{tabular}} & 1 & \multicolumn{1}{c}{ do } & \multicolumn{1}{c}{ Lote } \\
& 2 & 3 & 4 & 5 & 6 \\
\hline \multirow{4}{*}{ Observações } & 7,298 & 5,220 & 0,110 & 2,212 & 0,282 & 1,722 \\
& 3,846 & 6,556 & 10,386 & 4,852 & 9,014 & 4,782 \\
& 2,434 & 0,608 & 13,434 & 7,092 & 4,458 & 8,106 \\
individuais & 9,566 & 11,788 & 5,510 & 9,288 & 9,446 & 0,758 \\
& 7,990 & $-0,892$ & 8,166 & 4,980 & 7,198 & 3,758 \\
\hline$y_{j .}$ & 6,2268 & 4,6560 & 7,5212 & 5,6848 & 6,0796 & 3,8252 \\
\hline \hline
\end{tabular}

\subsubsection{Análise Clássica do Problema}

Utilizando a metodologia desenvolvida no capítulo 1, desenvolvemos uma análise clássica do problema.. Primeiramente obtemos a tabela de análise de variância (ver tabela (6.7)) apropriada para o problema.

Estimadores clássicos usuais não viciados de $\sigma_{1}^{2}$ e $\sigma_{2}^{2}$, de acordo com (1.10), são dados por $\hat{\sigma}_{1}^{2}=14,9459$ e $\hat{\sigma}_{2}^{2}=-1,3219$, e portanto, nesse caso temos o problema que citamos no início desse trabalho, que é a obtenção de estimativas negativas para o componente de variância $\sigma_{2}^{2}$. 
Tabela 6.7. Análise de variância para o exemplo 2 .

\begin{tabular}{||l|l|l|l||}
\hline Fonte de Var. & S.Q. & G.L. & Q.M. \\
\hline Lotes & $S_{2}=41,6816$ & $v_{2}=5$ & $m_{2}=8,3363$ \\
Análises & $S_{1}=358,7014$ & $v_{1}=24$ & $m_{1}=14,9459$ \\
Total & 400,3830 & 29 & \\
\hline
\end{tabular}

Intervalos de confiança $95 \%$ baseados nas distribuições das somas de quadrados $S_{1}$ e $S_{2}(\operatorname{ver}(1.20)$ e $(1.21))$ para $\sigma_{1}^{2}$ e para a razão $\sigma_{2}^{2} / \sigma_{1}^{2}$ são $(9,1124 ; 28,9247)$ e $(-0,1646 ; 0,5003)$, respectivamente. Observamos que nesse caso, também temos problema com o intervalo de confiança para a razão $\sigma_{2}^{2} / \sigma_{1}^{2}$, uma vez que o mesmo inclui valores negativos.

Os estimadores de máxima verossimilhança de $\sigma_{1}^{2}$ e $\sigma_{2}^{2}$ são dados por $\hat{\sigma}_{1}^{2}=14,9459$ e $\hat{\sigma}_{2}^{2}=-1,6331$, e com base na distribuição Normal assintótica desses estimadores, intervalos de confiança $95 \%$ são dados por $(6,4895 ; 23,4023)$ e $(-3,9423 ; 0,6761)$, para $\sigma_{1}^{2}$ e $\sigma_{2}^{2}$ respectivamente. Novamente verificamos a existência de valores negativos no intervalo confiança para $\sigma_{2}^{2}$.

\subsubsection{Análise Bayesiana para o Exemplo 2}

Considerando os dados da tabelas (6.6), construímos o gráfico da densidade a posteriori marginal aproximada para $\sigma_{1}^{2}(3.13)$ (ver figura 6.10), obtida pelo método de aproximação de Laplace. A moda dessa densidade a posteriori, de acordo com (3.14), é dadá por $\tilde{\sigma}_{1}^{2}=13,7962$.

Os gráficos das densidades a posteriori marginais aproximadas por Laplace para o parâmetro $\sigma_{2}^{2}$ (3.16) e (3.17), depois de levarmos em conta que esse parâmetro deve assumir apenas valores positivos, são mostrados na figura (6.11). Observamos que essa restrição provoca um "corte" nas curvas das densidades, e as modas a posteriori nesse caso são dadas por $\tilde{\sigma}_{2}^{2}=0$. 
Utilizando métodos de integração numérica obtemos um intervalo de credibilidade $95 \%$ aproximado para $\sigma_{1}^{2}$ dado por $(9,1125 ; 28,9250)$; para $\sigma_{2}^{2}$ esses intervalos são $(0 ; 5,6645)$ e $(0 ; 6,6620)$, de acordo com as densidades a priori (3.3) e (3.6) respectivamente.

Para a razão de variâncias $\sigma_{2}^{2} / \sigma_{1}^{2}$, intervalos de credibilidades $95 \%$ aproximados, obtidos a partir das densidades a posteriori marginais para W (3.19) e (3.21) (ver figura (6.12)), são dados por $(0 ; 0,4635)$ e $(0 ; 0,6052)$ de acordo com as densidades a priori (3.3) e (3.6) respectivamente.

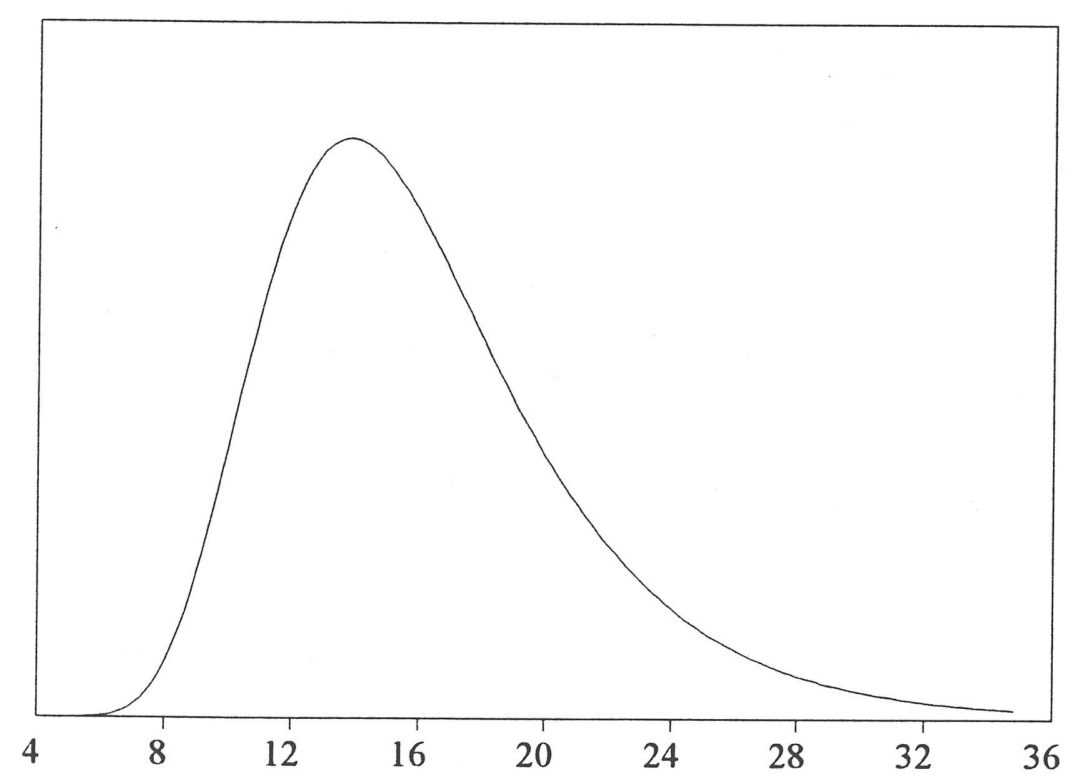

Figura 6.10 - Gráfico da densidade a posteriori marginal para $\sigma_{1}^{2}$. 


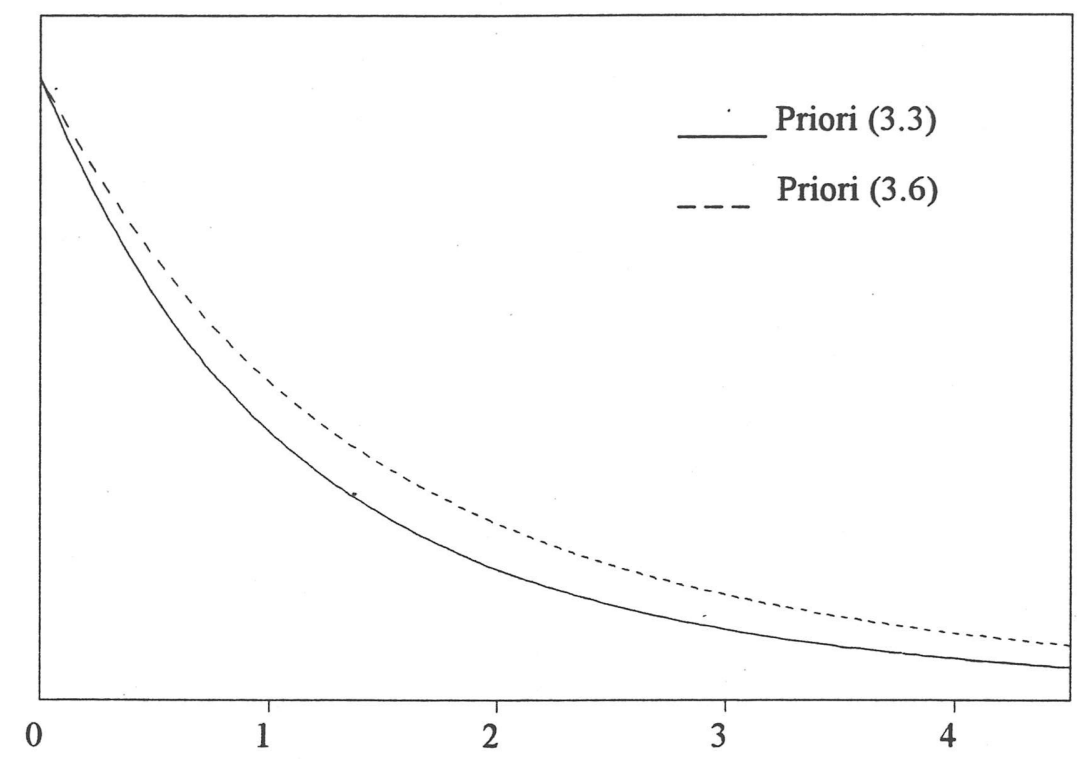

Figura 6.11 - Gráfico das densidades a posteriori marginais para $\sigma_{2}^{2}$.

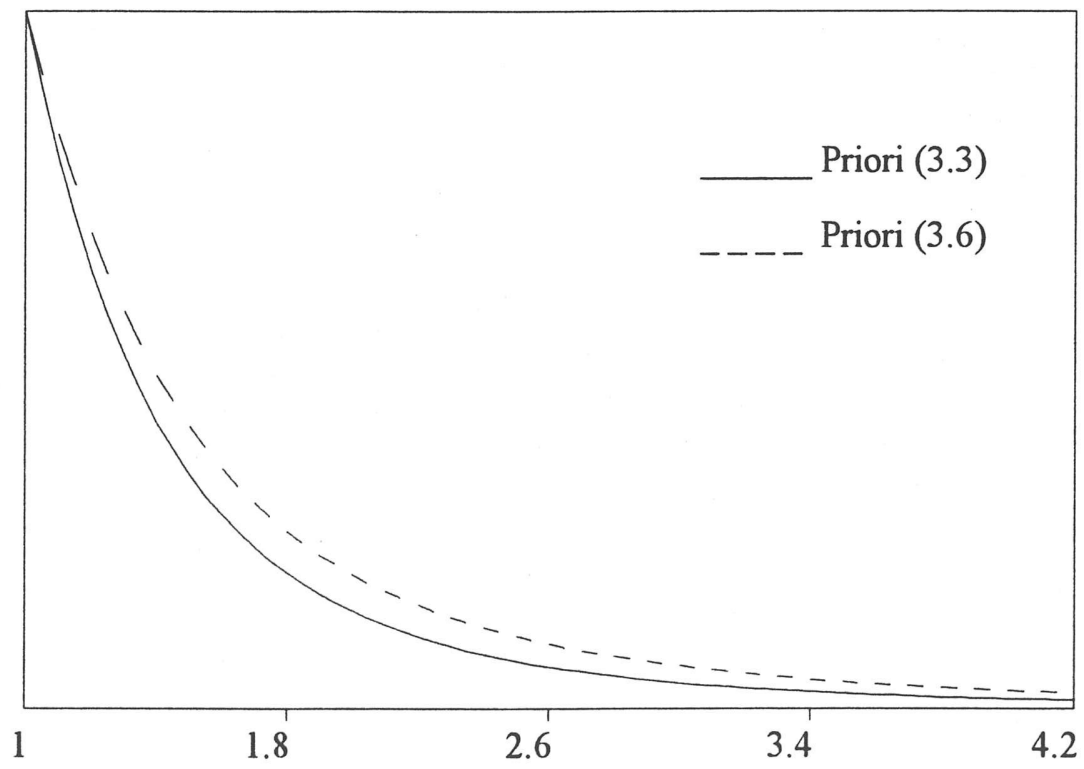

Figura 6.12 - Gráfico da densidade a posteriori marginal para W. 
Um valor de previsão para a média de um grupo futuro de observações $\dot{y}_{21}$, de acordo com a moda da densidades preditiva (3.24) ou (3.25), é dado por $y_{21 .}=5,66$. Novamente observamos que os resultados de previsão baseados nessas densidades preditivas são muito próximos para qualquer densidade a priori considerada (observar figura (6.13)).

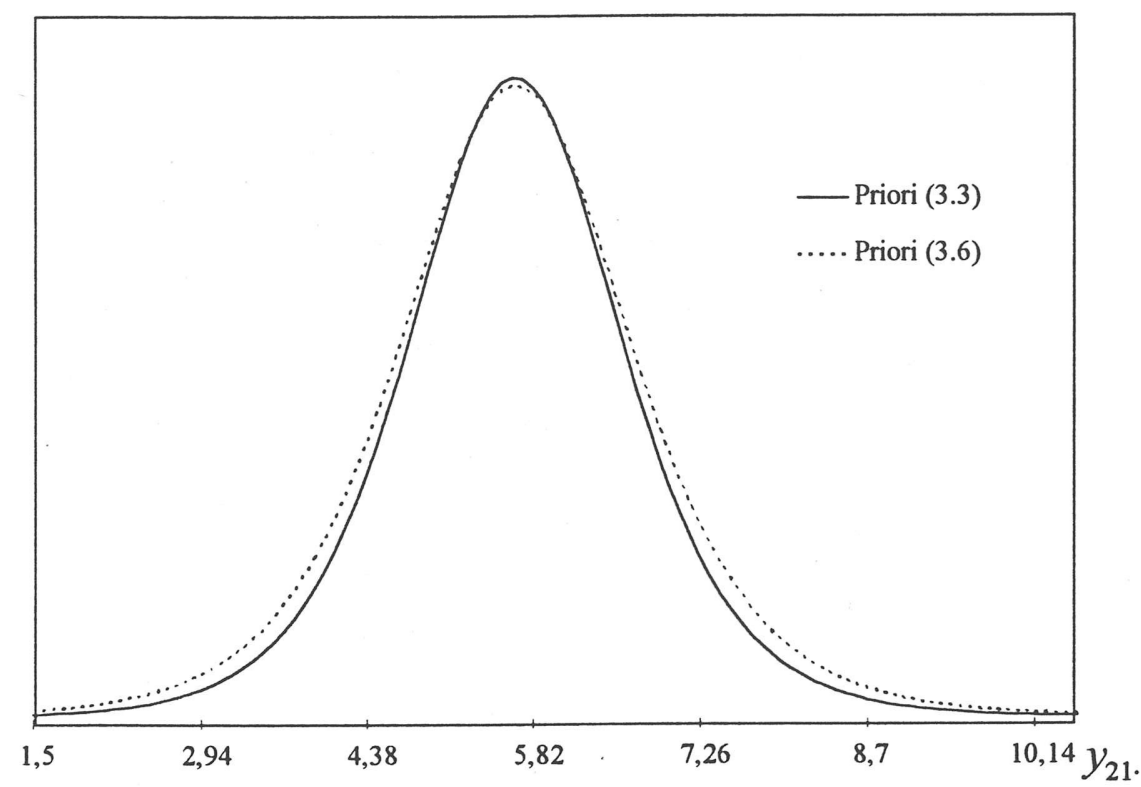

Figura 6.13 - Densidade preditiva para a média de um grupo futuro.

\subsubsection{Comparação entre as Estimativas Obtidas}

$\mathrm{Na}$ tabela (6.8) temos um resumo dos estimadores obtidos via análise de variância, máxima verossimilhança e as modas das densidades a posteriori marginais para $\sigma_{1}^{2}$ e $\sigma_{2}^{2}$. 
Tabela 6.8. Estimadores clássicos e modas a posteriori para os parâmetros $\sigma_{1}^{2}$ e $\sigma_{2}^{2}$.

\begin{tabular}{||l|l|c|c||}
\hline Parâmetro & $\begin{array}{c}\text { Estimador de } \\
\text { Análise de } \\
\text { Variância }\end{array}$ & $\begin{array}{c}\text { Estimadores de } \\
\text { Máxima Veross. }\end{array}$ & $\begin{array}{c}\text { Moda a } \\
\text { posteriori }\end{array}$ \\
\hline$\sigma_{1}^{2}$ & $\hat{\sigma}_{1}^{2}=14,9459$ & $\hat{\sigma}_{1}^{2}=14,9459$ & $\tilde{\sigma}_{1}^{2}=13,7962$ \\
\hline$\sigma_{2}^{2}$ & $\hat{\sigma}_{2}^{2}=-1,3219$ & $\hat{\sigma}_{2}^{2}=-1,6331$ & $\tilde{\sigma}_{2}^{2}=0$ \\
\hline
\end{tabular}

Da tabela (6.8) vemos que os estimadores clássicos de $\sigma_{2}^{2}$, e também a moda a posteriori, não se mostram como sendo satisfatórios para estimar esse parâmetro um vez que os valores obtidos estão distantes do verdadeiro valor.

Na tabela (6.9) resumimos os intervalos de confiança e de credibilidade obtidos para os parâmetros $\sigma_{1}^{2}$ e $\sigma_{2}^{2}$.

Tabela 6.9. Intervalos de confiança e de credibilidade $95 \%$ para os parâmetros $\sigma_{1}^{2}$ e $\sigma_{2}^{2}$.

\begin{tabular}{|c|l|c|c|c||}
\hline $\begin{array}{c}\text { Tipo de } \\
\text { inter. }\end{array}$ & $\begin{array}{l}\text { Procedi- } \\
\text { mento }\end{array}$ & Parâmetro $\sigma_{1}^{2}$ & Parâmetro $\sigma_{2}^{2}$ & Razão $\sigma_{2}^{2} / \sigma_{1}^{2}$ \\
\hline $\begin{array}{c}\text { Norm. Assin. } \\
\text { Int. de Conf. }\end{array}$ & Exato & $(6,4895 ; 23,4023)$ & $(-3,9423 ; 0,6761)$ & - \\
\hline $\begin{array}{c}\text { S.Q. } \\
S_{1} \mathrm{e} S_{2}\end{array}$ & Exato & $(9,1124 ; 28,9247)$ & - & $(-0,1646 ; 0,5003)$ \\
\hline $\begin{array}{c}\text { Inter. de } \\
\text { Credib. }\end{array}$ & Integração & $\begin{array}{c}(9,1125 ; 28,9250)^{1} \\
(9,1125 ; 28,9250)^{2}\end{array}$ & $\begin{array}{c}(0 ; 5,6645)^{1} \\
(0 ; 6,6620)^{2}\end{array}$ & $\begin{array}{c}(0 ; 0,4635)^{1} \\
(0 ; 0,6052)^{2}\end{array}$ \\
\hline
\end{tabular}

NOTA: (1) indica que o intervalo de credibilidade foi obtido utilizando-se a densidade a priori (3.3) e (2) a densidade a priori (3.6).

Observando a tabela (6.9) vemos que o intervalo de credibilidade aproximado e o intervalo de confiança de $\sigma_{1}^{2}$, obtido a partir da distribuição amostral de $S_{1}$, são praticamente iguais. Vemos também, a grande vantagem de trabalhar com inferência Bayesiana, uma vez que os intervalos de credibilidade para $\sigma_{2}^{2}$ e para $\sigma_{2}^{2} / \sigma_{1}^{2}$ não apresentam valores negativos. 


\subsubsection{Verificação da Normalidade das Densidades a Posteriori}

Construindo contornos aproximados das densidades a posteriori (3.10) e (3.11) (ver figura (6.14)) verificamos que eles não se aproximam de elipses. Vemos também que nesse caso a moda a posteriori irrestrita estará fora do espaço de definição dos parâmetros para qualquer densidade a priori. Portanto para o cálculo das aproximações de Laplace para os momentos a posteriori devemos levar em conta que existe uma restrição no espaço paramétrico, uma vez que os máximos das funções envolvidas estarão fora do espaço paramétrico.
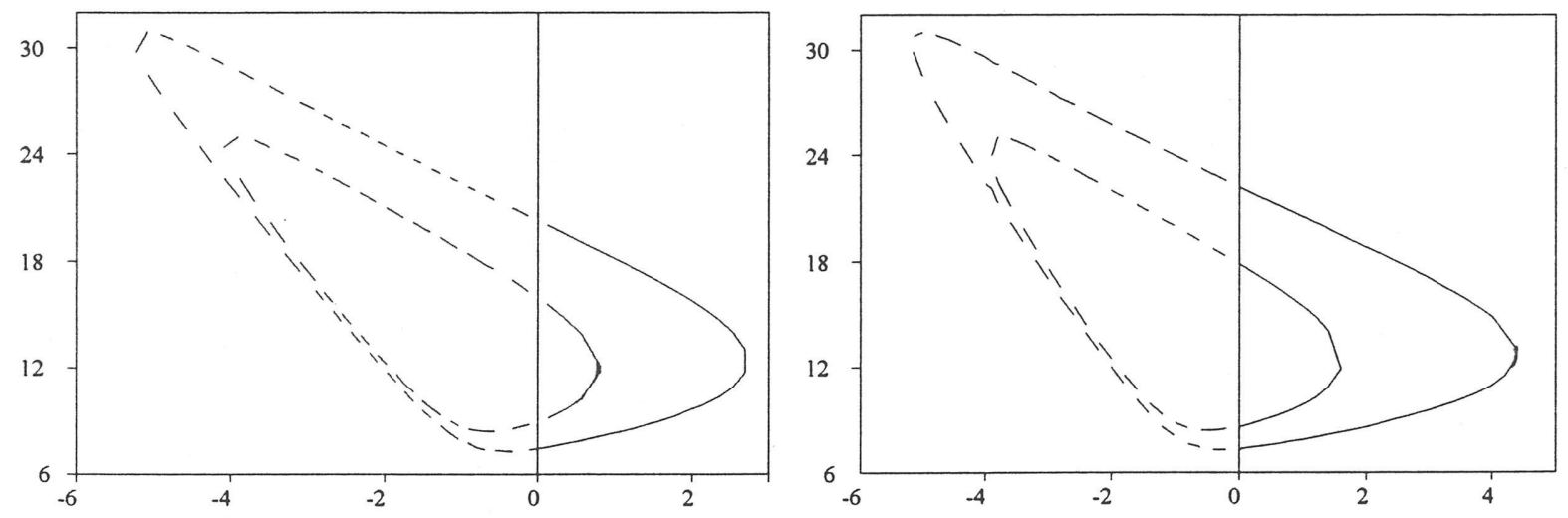

Figura 6.14 -Contornos das densidades a posteriori conjuntas para $\sigma_{1}^{2}$ e $\sigma_{2}^{2}$.

Para esse exemplo, também avaliamos a normalidade das densidades a posteriori "profile" para $\sigma_{1}^{2}$ e $\sigma_{2}^{2}$ através do método gráfico T-Plot de Hills e Smith. Podemos observar que, para as duas densidades a priori consideradas, as formas nesses gráficos (observar figuras (6.15) e (6.16)) são bastante curvas indicando novamente a não normalidade das densidades a posteriori para esses parâmetros.

As medidas de não-normalidade de Kass e Slate para esse conjunto de dados são: 5,2309 e 5,8023, de acordo com as densidades a priori (3.3) e (3.6) respectivamente. 

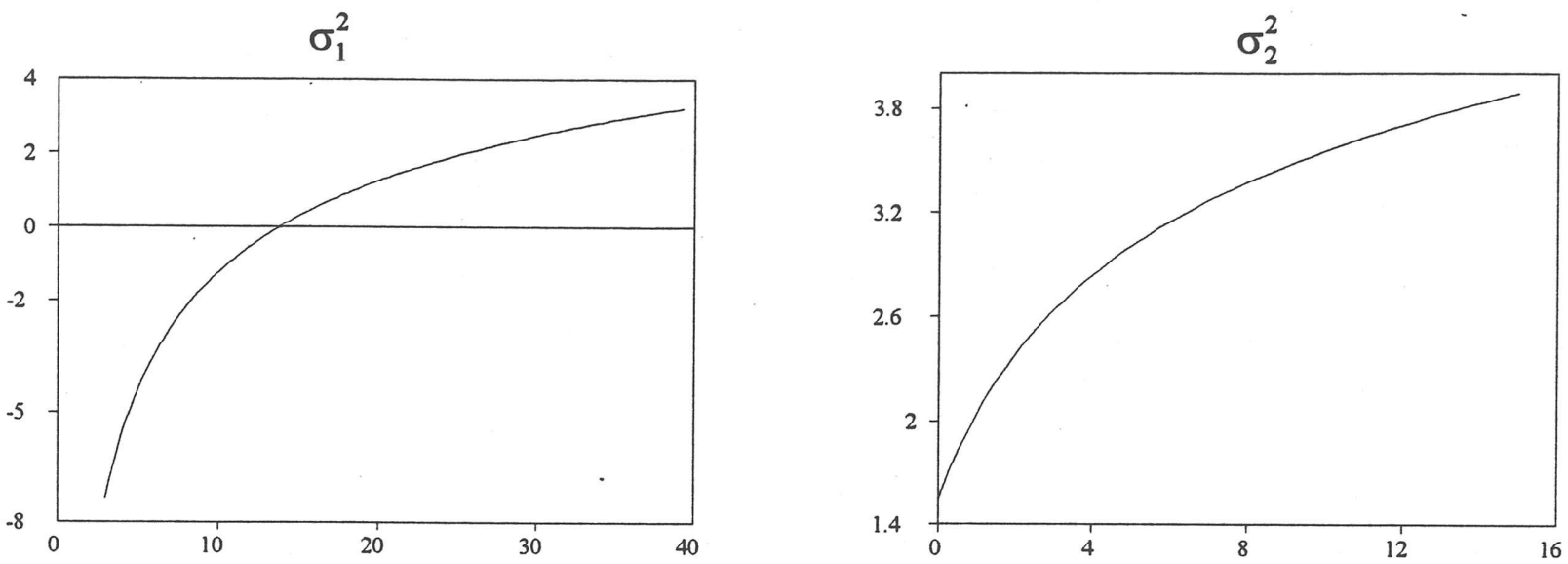

Figura 6.15 - T-plot's das densidades "profile" considerando a densidade a priori (3.3).
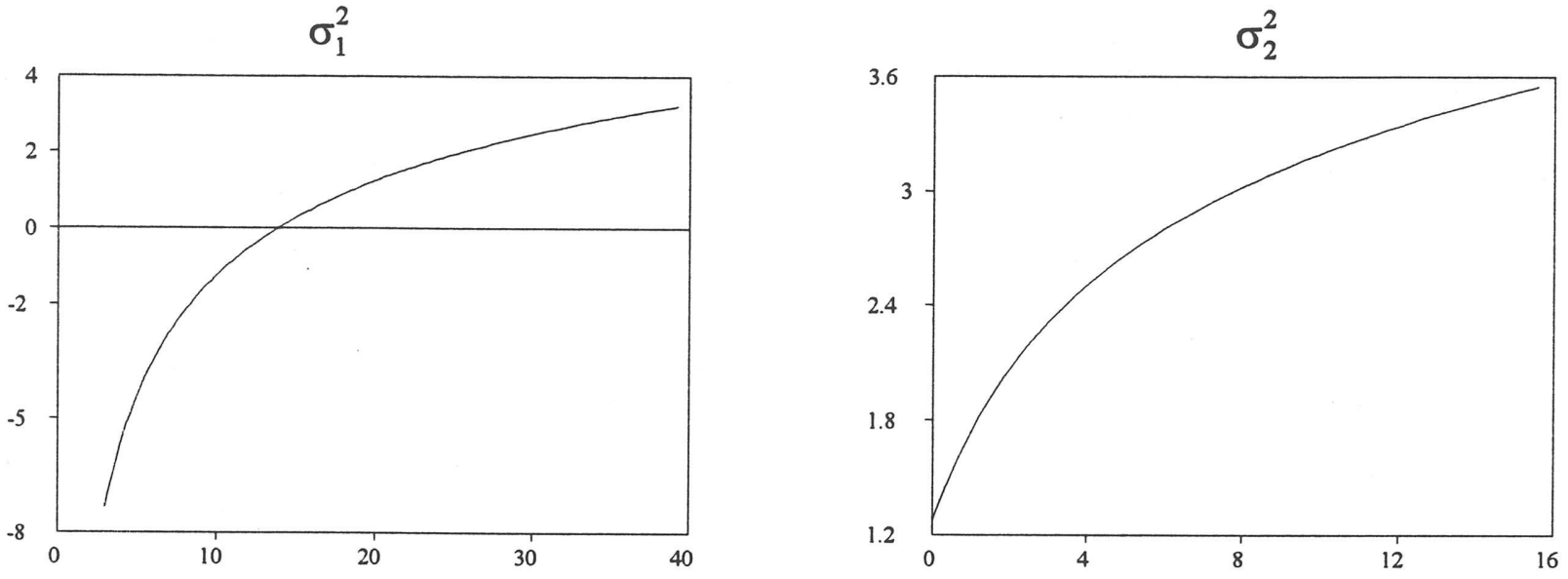

Figura 6.16 - T-plot's das densidades "profile" considerando a densidade a priori (3.6).

Para obter uma reparametrização que melhore a normalidade de densidade a posteriori conjunta (3.11) utilizamos a medida de não-normalidade de Kass e Slate $m^{2} \bar{B}^{2}$ para encontrar um valor de $\lambda$ em (3.45) que seja próximo de zero. Os valores obtidos são dados na tabela (6.10). Observamos que o valor mais apropriado é dado por $\lambda=-0,7$, pois é o que fornece o valor de $m^{2} \bar{B}^{2}$ menor. Portanto uma reparametrização que leva a uma densidade a posteriori mais próxima da normal é dada por

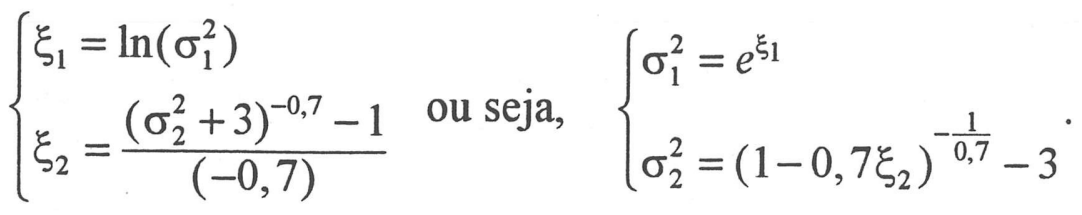


De acordo com essa parametrização e com o conjunto de dados temos que a densidade a posteriori conjunta para $\left(\xi_{1}, \xi_{2}\right)$ é dada por

$\pi\left(\xi_{1}, \xi_{2} / y\right) \propto\left(e^{\xi_{1}}\right)^{-12}\left\{A\left(\xi_{1}, \xi_{2}\right)\right\}^{-3,5}\left(1-0,7 \xi_{2}\right)^{-\frac{1}{0,7}-1} \exp \left\{-\frac{1}{2}\left[\frac{41,6816}{A\left(\xi_{1}, \xi_{2}\right)}+\frac{358,7014}{e^{\xi_{1}}}\right]\right\}$,

onde, $A\left(\xi_{1}, \xi_{2}\right)=e^{\xi_{1}}+5\left(1-0,7 \xi_{2}\right)^{-\frac{1}{0,7}}-15$.

Tabela 6.10 - Medidas de não- normalidade $m^{2} \bar{B}^{2}$ considerando vários valores de $\lambda$ em (3.45).

\begin{tabular}{||cc||}
\hline$\lambda$ & $m^{2} \bar{B}^{2}$ \\
\hline-1 & 1,14452 \\
$-0,9$ & 0,97065 \\
$-0,8$ & 0,88447 \\
$-0,7$ & 0,85714 \\
$-0,6$ & 0,86932 \\
$-0,5$ & 0,90803 \\
$-0,4$ & 0,96457 \\
$-0,2$ & 1,11049 \\
0 & 1,51680 \\
0,2 & 1,48078 \\
0,4 & 1,71414 \\
0,6 & 2,01346 \\
0,8 & 2,43770 \\
1 & 3,09376 \\
\hline
\end{tabular}




\subsubsection{Momentos a Posteriori Exatos e Aproximados}

De forma análoga ao exemplo 1 , na tabela (6.11) resumimos os momentos a posteriori para $\sigma_{1}^{2}$ e $\sigma_{2}^{2}$ e para funções dos mesmos obtidos no capítulo 3. Mostramos também as percentagem dos erros das aproximações, em relação as fórmulas exatas.

Tabela 6.11 - Momentos a posteriori exatos e aproximados para funções $\sigma_{1}^{2}$ e $\sigma_{2}^{2}$.

\begin{tabular}{||l|l|l|l||}
\hline \multirow{2}{*}{} & \multirow{2}{*}{ Valor Exato } & \multicolumn{2}{|c|}{$\begin{array}{c}\text { Aproximação de Laplace na } \\
\text { Parametrização }\end{array}$} \\
\cline { 3 - 4 } & $\left(\sigma_{1}^{2}, \sigma_{2}^{2}\right)$ & $\left(\xi_{1}, \xi_{2}\right)$ \\
\hline$E\left(\sigma_{1}^{2} / y\right)$ & $\mathbf{1 4 , 2 6 4 8}$ & $\mathbf{1 3 , 1 6 9 3}$ & $\mathbf{1 5 , 1 6 4 9}$ \\
& & $(8,32 \%)$ & $(6,31 \%)$ \\
\hline$E\left(\sigma_{12}^{2} / y\right)$ & $\mathbf{2 8 , 8 5 1 5}$ & $\mathbf{4 0 , 3 6 6 5}$ & $\mathbf{3 2 , 4 0 1 4}$ \\
& & $(39,91 \%)$ & $(12,30 \%)$ \\
\hline$E\left(\frac{\sigma_{12}^{2}}{\sigma_{1}^{2}} / y\right)$ & $\mathbf{2 , 0 5 5 5}$ & $\mathbf{3 , 2 2 2 9}$ & $\mathbf{2 , 2 5 7 2}$ \\
& & $(56,8 \%)$ & $(9,81 \%)$ \\
\hline
\end{tabular}

De acordo com a tabela (6.11) podemos observar que esse conjunto de dados apresenta maiores problemas na utilização de métodos aproximados, pois os erros cometidos quando utilizamos o método de aproximação de Laplace, na parametrização original $\left(\sigma_{1}^{2}, \sigma_{2}^{2}\right)$, são grandes. Como já era esperado, na parametrização $\left(\xi_{1}, \xi_{2}\right)$ verificamos um aumento na precisão dos resultados, porém não tão grande como no exemplo anterior. 


\subsection{Um Exemplo Considerando o Modelo com Mistura de Normais}

Considere o conjunto de dados simulados da tabela (6.12), introduzido por Tiao e Ali (1971), assumindo o modelo de efeitos aleatórios (1.8) com densidade (1.32) para o efeito aleatório $e_{j}$, onde $\mu=0 \sigma_{1}^{2}=1, \sigma_{2}^{2}=4, \phi=1$ e $\lambda=3$.

Tabela 6.12 - Médias de grupo $y_{(J+1)}$. ordenadas (dados simulados $\operatorname{com} \mu=0 \quad \sigma_{1}^{2}=1, \sigma_{2}^{2}=4, \phi=1$ e $\lambda=3, J=20$ e $K=3$ ).

\begin{tabular}{||c|cccccc||}
\hline \hline Grupo $j$ & 1 & 2 & 3 & 4 & 5 & 6 \\
& $-3,682$ & $-2,057$ & $-1,780$ & $-1,280$ & $-0,797$ & $-0,671$ \\
\hline Grupo $j$ & 7 & 8 & 9 & 10 & 11 & 12 \\
& $-0,646$ & $-0,471$ & $-0,436$ & $-0,401$ & $-0,378$ & 0,000 \\
\hline Grupo $j$ & 13 & 14 & 15 & 16 & 17 & 18 \\
& 0,112 & 0,791 & 0,923 & 1,571 & 1,712 & 4,223 \\
\hline Grupo $j$ & 19 & 20 & & & & \\
& 6,415 & 7,072 & & & & \\
\hline
\end{tabular}

Dos dados da tabela (6.12), temos $J=20$ e $K=3, v_{1}=J(K-1)=40, J K y_{. .}=$ $\sum_{j} \sum_{k} y_{j k}=0,5131, \quad m_{1}=1,1525, \quad v_{1} m_{1}=S_{1}=46,1$. O logaritmo da função de verossimilhança (5.2) é dado por

$$
\ln L\left(\sigma_{1}^{2}, \sigma_{2}^{2}, \phi, \lambda / y\right) \propto-20 \ln \left(\sigma_{1}^{2}\right)-\frac{23,05}{\sigma_{1}^{2}}+\sum_{j=1}^{20} \ln \left(A_{1 j}+A_{2 j}\right),
$$

onde $A_{1 j}$ e $A_{2 j}, j=1, \ldots, J$ são dados em (5.1). Os estimadores de máxima verossimilhança para $\sigma_{1}^{2}, \sigma_{2}^{2}, \phi$ e $\lambda$ (ver tabela (6.13)) são dados por $\hat{\sigma}_{1}^{2}=1,1546$, $\hat{\sigma}_{2}^{2}=3,1355, \hat{\phi}=1$ e $\hat{\lambda}=3,7625$. 
Tabela 6.13 - Estimadores de máxima verossimilhança para $\sigma_{1}^{2}, \sigma_{2}^{2}$ e $\lambda$ com $\phi$ conhecido.

\begin{tabular}{||l|llcc||}
\hline \hline$\phi$ & $\hat{\sigma}_{1}^{2}$ & $\hat{\sigma}_{2}^{2}$ & $\hat{\lambda}$ & $\ln L\left(\hat{\sigma}_{1}^{2}, \hat{\sigma}_{2}^{2}, \phi, \hat{\lambda} / y\right)$ \\
\hline-1 & 1,1525 & 6,6456 & 1,0001 & $-52,34026$ \\
0 & 1,1525 & 3,8737 & 4,0757 & $-51,93104$ \\
1 & 1,1546 & 3,1355 & 3,7625 & $-50,41699$ \\
\hline
\end{tabular}

Usando segundas derivadas numéricas de $\sum_{j=1}^{20} \ln \left(A_{1 j}+A_{2 j}\right)$ localmente nos estimadores de máxima verossimilhança $\hat{\sigma}_{1}^{2}, \hat{\sigma}_{2}^{2}, \hat{\phi}$ e $\hat{\lambda}$ (observar Apêndice A), obtemos a matriz de informação de Fisher observada dado $\phi=1$,

$$
I=\left(\begin{array}{rrr}
15,05601 & 0,16206 & -0,15655 \\
0,16206 & 0,38366 & -0,06089 \\
-0,15655 & -0,06089 & 0,37574
\end{array}\right) .
$$

Considerando a distribuição usual normal assintótica para os estimadores de máxima verossimilhança $\left(\hat{\sigma}_{1}^{2}, \hat{\sigma}_{2}^{2}, \hat{\lambda}\right)$ dado $\phi=1$, obtemos intervalos de confiança $95 \%$ para $\sigma_{1}^{2}, \sigma_{2}^{2}$ e $\lambda$ dados por $0,64715<\sigma_{1}^{2}<1,6617,-0,0757<\sigma_{2}^{2}<6,3467$, e $0,5180<\lambda<7,0070$.

$\mathrm{Na}$ tabela (6.14), temos as densidades a posteriori marginais aproximadas (5.6) considerando diferentes escolhas para a densidade a priori $\pi_{0}(\phi, \lambda)$. Também consideramos o uso do método de derivadas numéricas para calcular o determinante da matriz Hessiana dada na aproximação de Laplace em (5.6), para cada valor de $\phi$. A moda das densidades a posteriori marginais para $\phi$ considerando diferentes densidades a priori são todas dadas por $\tilde{\phi}=1$. 
Tabela 6.14 - Densidade a posteriori marginal aproximada (5.6) para $\phi$ considerando diferentes densidades a priori $\pi_{0}(\phi, \lambda)$

\begin{tabular}{||l|r|l|}
\hline$\pi_{0}(\phi, \lambda)=\pi_{01}(\phi) . \pi_{02}(\lambda)$ & \multicolumn{1}{|c|}{$\phi$} & $\pi(\phi / y)$ \\
\hline$\pi_{01}(\phi)=1 / 3, \phi=-1,0,1$ & -1 & 0,0658 \\
$\pi_{02}(\lambda) \propto$ constante & 0 & 0,2194 \\
& 1 & 0,7148 \\
\hline$\pi_{01}(\phi)=1 / 3, \phi=-1,0,1$ & -1 & 0,2126 \\
$\pi_{02}(\lambda) \propto 1 / \lambda$ & 0 & 0,1738 \\
& 1 & 0,6136 \\
\hline$\pi_{01}(\phi)=1 / 3, \phi=-1,0,1$ & -1 & 0,0134 \\
$\pi_{02}(\lambda) \propto \exp \left\{-\frac{1}{2}(\lambda-3)^{2}\right\}$ & 0 & 0,1846 \\
& 1 & 0,8020 \\
\hline$\pi_{01}(\phi)=1 / 2, \phi=1$ & -1 & 0,1317 \\
$\pi_{01}(\phi)=1 / 4, \phi=0$ & & 0,1077 \\
$\pi_{01}(\phi)=1 / 4, \phi=-1$ & 0 & 0,7606 \\
$\pi_{02}(\lambda)=1 / \lambda$ & 1 & \\
\hline
\end{tabular}

Assumindo $\phi=1$ conhecido, temos na figura (6.17), o gráfico da densidade a posteriori marginal aproximada (5.10) para $\lambda$ considerando algumas escolhas diferentes de densidades a priori para $\sigma_{1}^{2}, \sigma_{2}^{2}$ e $\lambda$ dado $\phi=1$ :
(i) $\pi_{1}\left(\sigma_{1}^{2}, \sigma_{2}^{2}, \lambda / \phi\right) \propto \lambda^{-1}\left(\sigma_{1}^{2}\right)^{-1}\left(\sigma_{1}^{2}+3 \sigma_{2}^{2}\right)^{-1}$,
(ii) $\pi_{2}\left(\sigma_{1}^{2}, \sigma_{2}^{2}, \lambda / \phi\right) \propto \exp \left\{-\frac{1}{8}(\lambda-3)^{2}\right\}\left(\sigma_{1}^{2}\right)^{-1}\left(\sigma_{1}^{2}+3 \sigma_{2}^{2}\right)^{-1}$,
(iii) $\pi_{3}\left(\sigma_{1}^{2}, \sigma_{2}^{2}, \lambda / \phi\right) \propto \exp \left\{-\frac{1}{2}(\lambda-3)^{2}\right\}\left(\sigma_{1}^{2}\right)^{-1}\left(\sigma_{1}^{2}+3 \sigma_{2}^{2}\right)^{-1}$. 
Utilizamos também segundas derivadas numéricas para calcular o determinante da matriz Hessiana em (5.10). A moda da densidade a posteriori está em torno de $\tilde{\lambda} \cong 3,5$ considerando todas as densidades a priori $\pi_{1}, \pi_{2}$, e $\pi_{3}$.

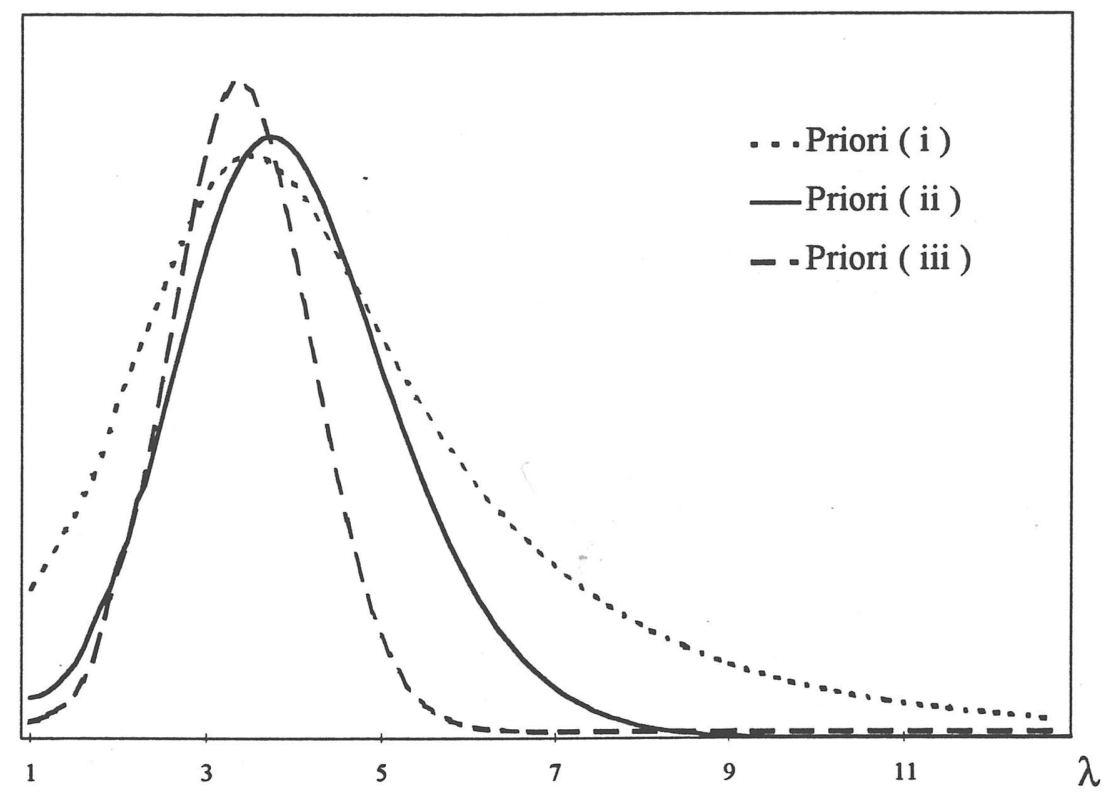

Figura 6.17 - Densidade a posteriori marginal aproximada para $\lambda$ assumindo $\phi=1$ conhecido.

Assumindo $\phi=1$ e $\lambda=3,5$, conhecidos, temos na figura (6.18), o gráfico da densidade a posteriori marginal aproximada (5.14) para $\sigma_{1}^{2}$ considerando a densidade a priori $\pi\left(\sigma_{1}^{2}, \sigma_{2}^{2} / \phi, \lambda\right) \propto\left(\sigma_{1}^{2}\right)^{-1}\left(\sigma_{1}^{2}+3 \sigma_{2}^{2}\right)^{-1}, \sigma_{1}^{2}, \sigma_{2}^{2}>0$.

Temos também, na figura (6.18), o gráfico da densidade a posteriori marginal aproximada para $\sigma_{1}^{2}(5.20)$ assumindo $\lambda=1$, isto é, uma distribuição normal para o efeito aleatório $e_{j}$. Observamos resultados muito próximos para $\sigma_{1}^{2}$ considerando normalidade ou não-normalidade de $e_{j}, j=1, \ldots, J$. 


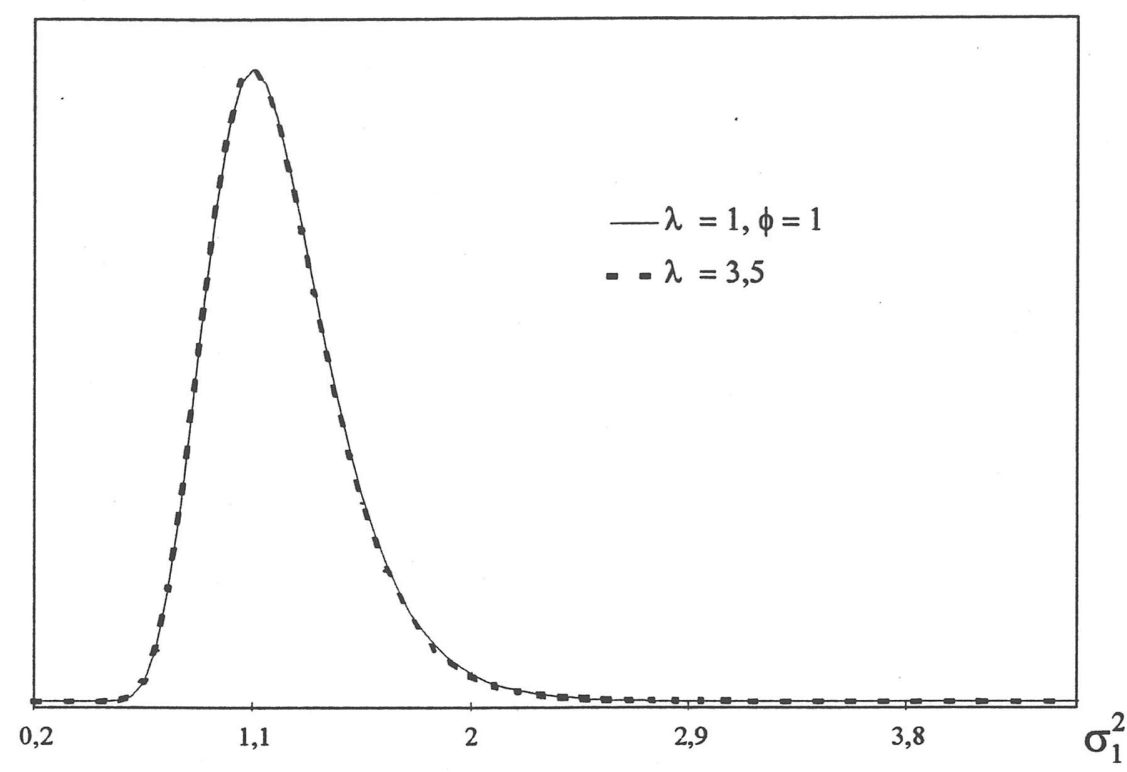

Figura 6.18 - Densidade posteriori marginal aproximada para $\sigma_{1}^{2}$.

Também, com $\phi=1$ e $\lambda=3,5$ conhecidos, e considerando a mesma priori nãoinformativa para $\sigma_{1}^{2}$ e $\sigma_{2}^{2}$, temos na figura (6.19) o gráfico da densidade a posteriori aproximada (5.16) para $\sigma_{2}^{2}$. Temos também na figura (6.19), o gráfico da densidade a posteriori aproximada (5.21) para $\sigma_{2}^{2}$ considerando $\lambda=1$. Nesse caso, observamos muita diferença nas inferências resultantes para $\sigma_{2}^{2}$ considerando normalidade e nãonormalidade para os efeitos aleatórios $e_{j}, j=1, \ldots, J$. É importante salientar que podemos considerar o uso dessas aproximações de Laplace para qualquer escolha de opinião a priori sobre $\sigma_{1}^{2}$ e $\sigma_{2}^{2}$. 


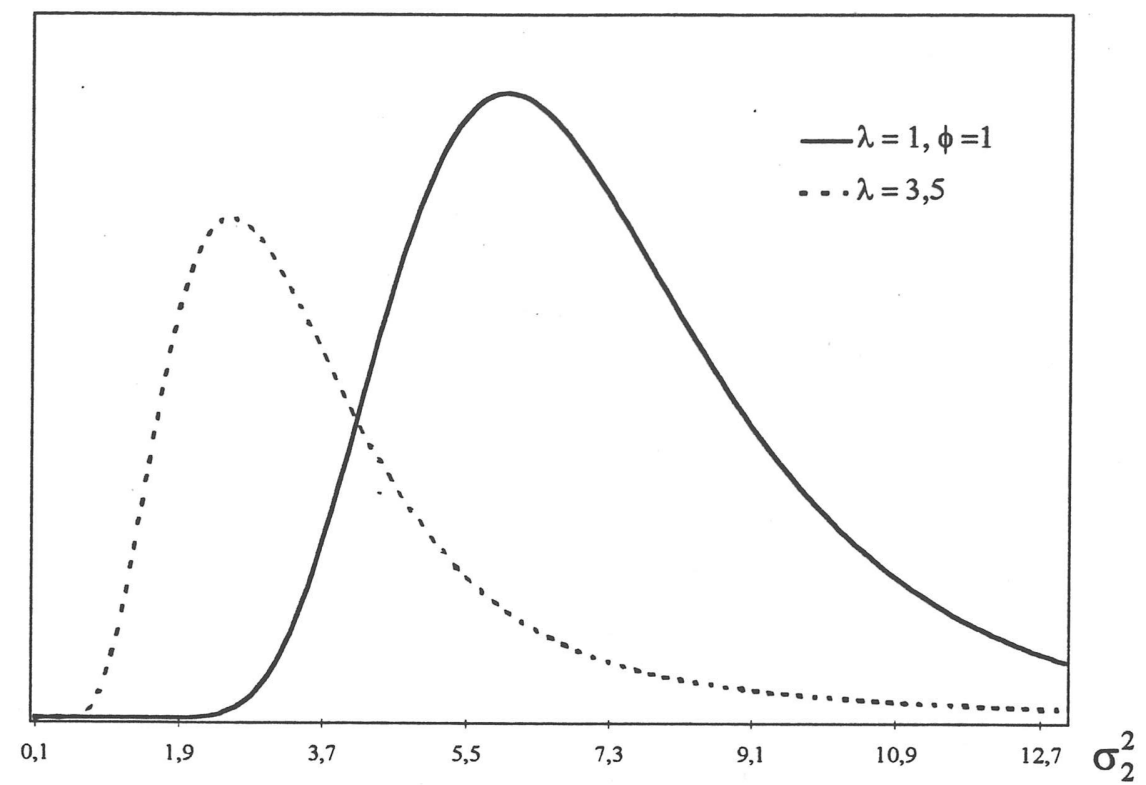

Figura 6.19 - Densidade a posteriori marginal aproximada para $\sigma_{2}^{2}$.

$\mathrm{Na}$ figura (6.20), temos o gráfico da densidade preditiva aproximada (5.18) para uma média de um grupo futuro $y_{(J+1)}$, considerando o conjunto de dados da tabela (6.12).

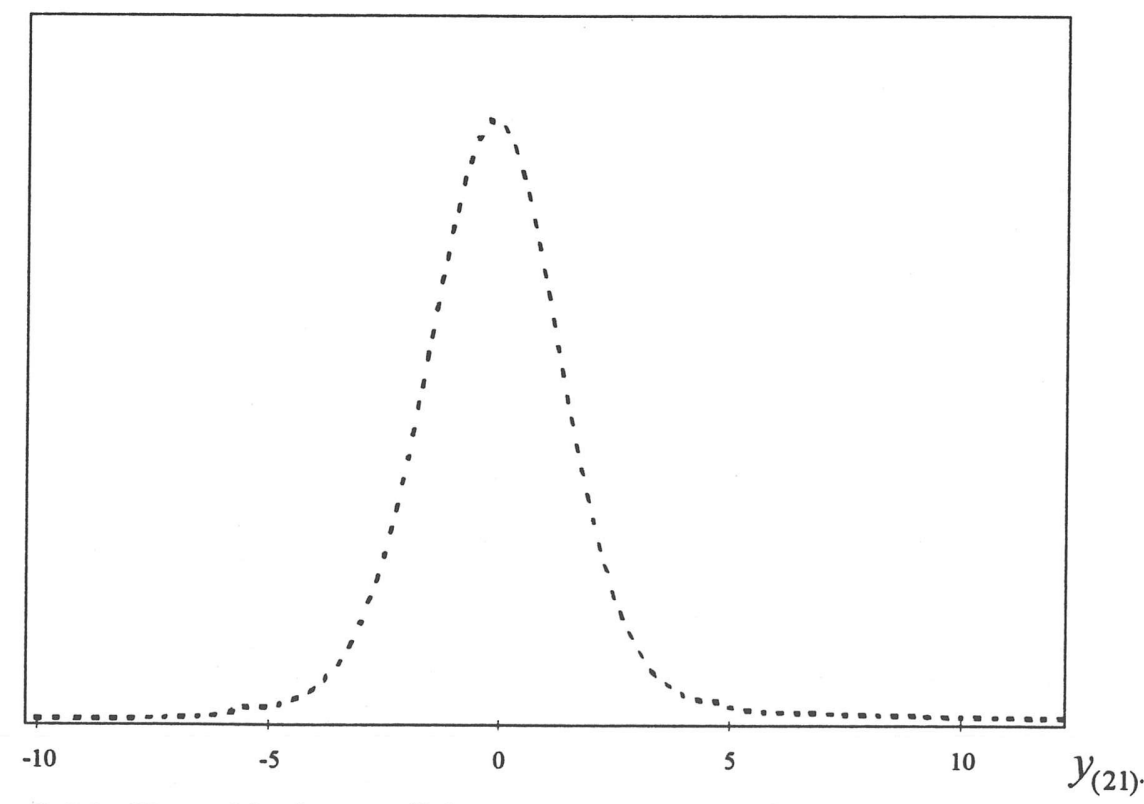

Figura 6.20- Densidade preditiva para uma média de um grupo futuro $y_{(J+1)}$. assumindo $\phi=1$ e $\lambda=3,5$. 
O uso do método de Laplace para aproximação de integrais pode ser justificado comparando-se a densidade a posteriori marginal integrada numericamente com a aproximação de Laplace. Assumindo os dados da tabela (6.12), com $\phi=1$ e $\lambda=3,5$ conhecidos, temos na figura (6.21) o gráfico da densidade a posteriori marginal para $\sigma_{1}^{2}$ considerando o método de Laplace e um procedimento numérico baseado na quadratura Gaussiana (Gauss-Hermite com $n=9$, raízes da equação polinomial de Hermite). Observamos que os resultados são muito próximos para ambos métodos de integração (ver também tabela (6.15)).

É importante salientar que a precisão da aproximação obtida usualmente depende de boa parametrização e dos dados, especialmente para pequenos tamanhos amostrais (ver por exemplo, Achcar e Smith, 1990).

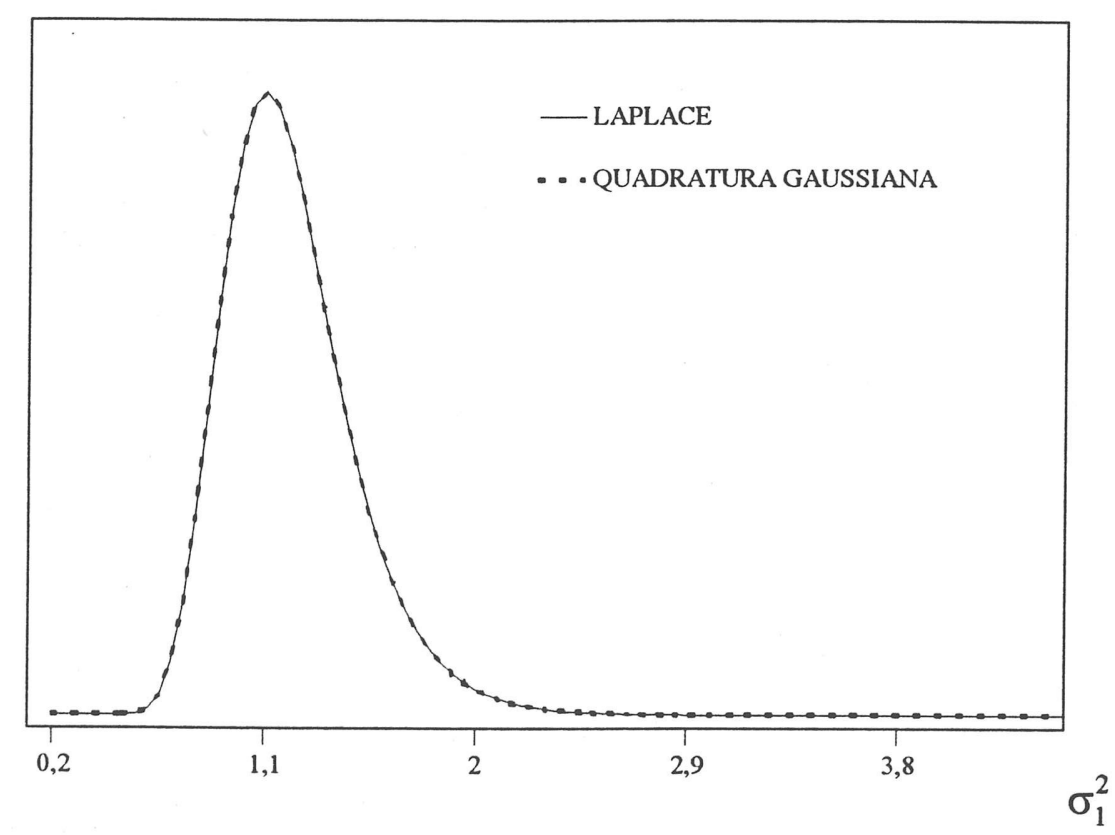

Figura 6.21 - Densidade a posteriori marginal para $\sigma_{1}^{2} \operatorname{com} \phi=1$ e $\lambda=3,5$ conhecidos. 


\begin{tabular}{||ccc||}
\hline$\sigma_{1}^{2}$ & & Quadratura \\
& Laplace & Guassiana \\
\hline 0,2 & 0,000000 & 0,000000 \\
0,3 & 0,000000 & 0,000000 \\
0,4 & 0,000000 & 0,000000 \\
0,5 & 0,000048 & 0,000048 \\
0,6 & 0,002223 & 0,002268 \\
0,7 & 0,021107 & 0,021536 \\
0,8 & 0,078374 & 0,079903 \\
0,9 & 0,162301 & 0,165197 \\
1,0 & 0,229981 & 0,233518 \\
1,2 & 0,232970 & 0,234881 \\
1,4 & 0,142280 & 0,142033 \\
1,6 & 0,067468 & 0,066518 \\
1,8 & 0,028189 & 0,027383 \\
2,0 & 0,011101 & 0,010601 \\
2,2 & 0,004277 & 0,004007 \\
2,4 & 0,001647 & 0,001511 \\
2,6 & 0,000642 & 0,000576 \\
2,8 & 0,000255 & 0,000223 \\
3,0 & 0,000104 & 0,000088 \\
3,2 & 0,000043 & 0,000036 \\
3,4 & 0,000018 & 0,000015 \\
3,6 & 0,000008 & 0,000006 \\
3,8 & 0,000003 & 0,000003 \\
4,0 & 0,000003 & 0,000001 \\
\hline
\end{tabular}

Tabela 6.15 - Densidade a posteriori marginal para $\sigma_{1}^{2} \operatorname{com} \phi=1$ e $\lambda=3,5$ conhecidos. 


\section{Capítulo 7}

\section{Conclusões e Considerações Futuras}

O uso de métodos de aproximação de integrais de Laplace é uma forma simples de se obter inferências Bayesianas precisas para modelos de componentes de variância. É importante salientar que o uso de uma reparametrização adequada pode levar a resultados com grande precisão. Também, observamos que a suposição de nãonormalidade para os efeitos aleatórios deve ser considerada em muitas aplicações, pois os resultados podem ser muito diferentes quando consideramos normalidade para os efeitos aleatórios.

Como algumas considerações futuras para pesquisa neste tem, destacamos:

(i) $\mathrm{O}$ uso de outros métodos de integração para a obtenção dos resultados Bayesianos, especialmente para um grande número de efeitos aleatórios.

(ii) Consideração de densidades a priori informativas para os parâmetros do modelo de componentes de variância, considerando normalidade e não-normalidade para os efeitos aleatórios. 
(iii) Uso de densidades preditivas para discriminar modelos de componentes de variância considerando diferentes distribuições de probabilidade para os efeitos aleatórios.

(iv) Desenvolver técnicas para achar o número de unidades num problema de estimação de componentes de variância, envolvendo custo e precisão. Como um caso particular, usar técnicas Bayesianas para alocar unidades em testes e amostras que minimizam o custo com uma precisão aceitável. 


\section{Referências Bibliográficas}

ABRAMOWITZ, E. E STEGUN, I. (1965). Handbook of Mathematical Function. Dover.

ACHCAR, J.A. (1994). Approximate Bayesian Analysis for Non-Normal Hierarchical Classification Model. Notas do ICMSC, Série Estatística, 11 - USP São Carlos.

ACHCAR, J.A. E FOGO, J.C. (1993). An Useful Reparametrization for the Reliability in the Weibull Case. Notas do ICMSC, Série Estatística, 009 - USP São Carlos.

ACHCAR, J.A. E PEGORIN, M.J. (1995). Laplace's Approximations for Posterior Expectations when the Mode is not in the Parameter Space. Notas do ICMSC, Série Estatística, 15 - USP São Carlos.

ACHCAR, J.A. E SMITH, A.F.M. (1990). Aspects of Reparametrization in Aproximate Bayesian Inference. Essays in Honour of G. A. Barnard - Ed J.Hodges, pp. 439-452 - Amesterdam, Nort Holland.

BATES, M.D. E WATTS, G.D. (1980). Relative Curvature Measures of Nonlinearity. Journal of the Royal Statistical Society, série B, v. 42, pp. 1-25

BOX, G.E.P. E COX, D.R. (1964). An Analysis of Transformations. Journal of the Royal Statistical Society, série B, B. 26, pp. 211-252. 
BOX, G.E.P, HUNTER, W.G. E HUNTER, J.S. (1978). Statistics for experimenters. New York, John Wiley \& Sons.

BOX, G.E.P E TIAO, G.C. (1973). Bayesian Inference in Statistical Analysis. Reading Addison-Wesley.

COHEN, A.C. (1967). Estimation in Mistures of Two Normal Distributions. Technonometrics, v. 9, pp. 15-28.

CONTE, S.D. (1977). Elementos de Análise Numérica. 3a Ed. Edit. Globo.

CONTE, S.D. E BOOR, C. DE (1972). Elementary Numerical Analysis: an Algorithmic Appoach. New York, MC Graw-Hill.

COX, D.R. E REID, N. (1987). Parameter Orthogonality and Approximate conditional Inference. Journal of the Royal Statistical Society, série B, v. 49, 1, pp. 1-39.

DAVIS, L.O. (1958). Statistical Methods in Research and Production. 3a Ed., London, Oliver and Boyd.

GELFAND, A.E. E SMITH, A.F. (1990). Sampling Based Approaches to Calculating Marginal Densities. Journal of the American Statistical Association, v. 85, pp. $398-409$.

HASSELBLAD, V. (1966). Estimation of Parameters for a Mixture of Normal Distributions. Technometrics, v. 8, pp. 431-444. 
HERBACH, L.H. (1959). Properties of Model II-Type Analysis of Variânce Tests. Ann. Math. Statist. v. 30, pp. 039- 959.

HILL, B.M. (1965). Inference About Variance Components in the One-Way Model. Journal of the American Statistical Association, v. 58, pp.918-932.

HILLS, S.E. E SMITH, A.F.M. (1993). Diagnostic Plots for Improved Parametrization in Bayesian Inference. Biometrika, v. 80, pp. 61-74.

KASS, R.E. E SLATE, E.H. (1992). Reparametrization and Diagnostics of Posterior Non-Normality. In Bayesian Statistics 4, Ed. J.M. Bernardo, J.O. Berger, A.P. Dawid and A.F.M. Smith, pp.289 - 306. Oxford University Press.

KASS, E., TIERNEY, L. E KADANE, K. J. (1990). The Validity of Posterior Expansions Based on Laplace's Method. Essays in Honor of G.A. Barnard, pp. 473-488. Hodger, Amsterdam.

KLOEK, T. E VAN DIJK, H.K. (1978). Bayesian Estimates of Equation System Parameters: an Aplication of Integration of Monte Carlo. Econometrica, v. 46, pp. $1-19$.

LEONARD, T., HSU, J.S.S. E TSUI, K.W. (1989). Bayesian Marginal Inference. Journal of the American Statistical Association, v. 84, pp. 1051 - 1058.

MONTGOMERY, D.C. (1976). Design and Analysis of Experiments. New York, Jonh Wiley \& Sons. 
MOOD, A.M., GRA YBILL, A.F. E BOES, C.D. (1974). Introduction to the Teory of Statistics. 3a Ed., New York, McGraw-Hill.

NAYLOR, J.C. E SMITH, A.F.M. (1982). Application of a Method for the Efficient Computation of Posterior Distributions. Applied Stastistics, v. 31, pp. 214-225.

PRESS, S.J. (1989). Bayesian Statistics: Principles, Models, and Applications. New York, John Wiley \& Sons.

SCHEFFE, H. (1961). The Analysis of variânce. New York. Jhon Wiley \& Sons.

SPROTT, D. A. (1973). Normal Likelihood and Their Relacion to Large Sample Theory of Estimation. Biometrika, v. 60,3, pp. 457 - 465.

SPROTT, D.A. (1980). Maximun Likelihood in Small Samples: Estimation in the Presence of Nuisance Parameters. Biometrika, v. 67, pp. 515-523.

THOMPSON, W.A.JR. (1962). The Problem of Negative Estimates of Variance Components. Ann. Math. Statist, v. 33, pp. 273 - 279.

THOMPSON, W.A.JR. (1963). Non - Negative Estimates of Variance components. Technometrics, v. 5, pp. 441-449.

TIAO, G.C. E ALI, M.M. (1971). Effect of Non-Normality on Inferences About Variance Components. Technometrics, v. 13, pp. 635 - 651. 
TIAO, G.C E BOX, G.E.P. (1967). Bayesian Analysis of a Three-Component Hierarchical Design Model. Biometrika, v. 54, pp. 109 -125.

TIAO, G.C. E TAN, W.Y. (1965). Bayesian Analysis of Randon-Effect Models in the Analysis of Variance. I. Posteriori distribution of Variance-Components. Biometrika, v. 52, pp. 37 - 53.

TIERNEY, L. E KADANE, J.B. (1986). Accurate Approximations for Posterior Moments and Marginal Densities. Journal of the American Statistical Association, v. 81, pp. 82-6.

TIERNEY, L., KASS, R.E. E KADANE, J.B. (1989). Fully Exponential Laplace Approximations to Expectations and Variances of Nonpositive Functions. Journal of the American Statistical Association, v. 84, pp. 710-716.

TITTERINGTON, D.M., SMITH, A.F.M. E MAKOV, U.E. (1985). Statistical Analysis of Finite Mixture distributions. New York: John Wiley \& Sons. 


\section{Apêndice A}

\section{Derivadas Numéricas}

A utilização de derivação numérica é uma alternativa viável quando a função que desejamos derivar apresenta uma forma bastante complicada (ver por exemplo, Conte, 1977; Conte de De Boor, 1972).

Neste trabalho em particular, utilizamos derivadas numéricas da função de verossimilhança (5.2), para obter os estimadores de máxima verossimilhança dados na tabela (6.13) para cada valor de $\phi$ fixado, e também para calcular as matrizes Hessianas nas aproximações de Laplace para o modelo com mistura de normais, apresentado no capítulo 5.

Uma possível aproximação que oferece boa precisão para a derivada de primeira ordem de uma função $g(x)$ no ponto $\left(x_{0}\right)$ (ver Conte, 1977) é dada por

$$
g^{\prime}\left(x_{0}\right) \approx \frac{g\left(x_{0}-h\right)-g\left(x_{0}+h\right)}{2 h},
$$

com $h$ deve ser suficientemente pequeno.

Para as derivadas de segunda ordem utilizamos a aproximação:

$$
\left.\frac{\partial^{2} g(x, y)}{\partial x^{2}}\right|_{\left(x_{0}, y_{0}\right)} \approx \frac{1}{4 h^{2}}\left[g\left(x_{0}+2 h, y_{0}\right)-2 g\left(x_{0}, y_{0}\right)-g\left(x_{0}-2 h, y_{0}\right)\right]
$$




$$
\begin{aligned}
& \left.\frac{\partial^{2} g(x, y)}{\partial x \partial y}\right|_{\left(x_{0}, y_{0}\right)} \approx \frac{1}{4 h^{2}}\left[g\left(x_{0}+h, y_{0}+h\right)-g\left(x_{0}+h, y_{0}-h\right)-\right. \\
& \left.g\left(x_{0}-h, y_{0}+h\right)-g\left(x_{0}-h, y_{0}-h\right)\right] \\
& \left.\frac{\partial^{2} g(x, y)}{\partial y^{2}}\right|_{\left(x_{0}, y_{0}\right)} \approx \frac{1}{4 h^{2}}\left[g\left(x_{0}, y_{0}+2 h\right)-2 g\left(x_{0}, y_{0}\right)-g\left(x_{0}, y_{0}-2 h\right)\right] .
\end{aligned}
$$

Para obter os estimadores de máxima verossimilhança de $\left(\sigma_{1}^{2}, \sigma_{2}^{2}, \lambda\right)$ para cada valor de $\phi$ fixado $(\phi=-1,0,1)$, (observar tabela 6.13) utilizamos o método de Newton Raphson para resolver o sistema de equações dado por

$$
\left\{\begin{array}{l}
\frac{\partial \ln L\left(\sigma_{1}^{2}, \sigma_{2}^{2}, \phi, \lambda / y\right)}{\partial \sigma_{1}^{2}}=-\frac{20}{\sigma_{1}^{2}}+\frac{23,05}{\left(\sigma_{1}^{2}\right)^{2}}+\frac{\partial g\left(\sigma_{1}^{2}, \sigma_{2}^{2}, \phi, \lambda\right)}{\partial \sigma_{1}^{2}}=0 \\
\frac{\partial \ln L\left(\sigma_{1}^{2}, \sigma_{2}^{2}, \phi, \lambda / y\right)}{\partial \sigma_{2}^{2}}=\frac{\partial g\left(\sigma_{1}^{2}, \sigma_{2}^{2}, \phi, \lambda\right)}{\partial \sigma_{2}^{2}}=0 \\
\frac{\partial \ln L\left(\sigma_{1}^{2}, \sigma_{2}^{2}, \phi, \lambda / y\right)}{\partial \lambda}=\frac{\partial g\left(\sigma_{1}^{2}, \sigma_{2}^{2}, \phi, \lambda\right)}{\partial \sigma_{1}^{2}}=0
\end{array},\right.
$$

onde

$$
\ln L\left(\sigma_{1}^{2}, \sigma_{2}^{2}, \phi, \lambda / y\right) \propto-20 \ln \left(\sigma_{1}^{2}\right)-\frac{23,05}{\sigma_{1}^{2}}+g\left(\sigma_{1}^{2}, \sigma_{2}^{2}, \phi, \lambda\right),
$$

onde $g\left(\sigma_{1}^{2}, \sigma_{2}^{2}, \phi, \lambda\right)=\sum_{j=1}^{20} \ln \left(A_{1 j}+A_{2 j}\right)$, com $A_{1 j}$ e $A_{2 j}$ dados em $(5.1) \mathrm{e}$ 


$$
\begin{aligned}
& \left.\frac{\partial g\left(\sigma_{1}^{2}, \sigma_{2}^{2}, \phi, \lambda\right)}{\partial \sigma_{1}^{2}}\right|_{\psi_{i}}=\frac{1}{2 h}\left[g\left(\sigma_{1 i}^{2}+h, \sigma_{2 i}^{2}, \phi, \lambda_{i} / y\right)-g\left(\sigma_{1 i}^{2}-h, \sigma_{2 i}^{2}, \phi, \lambda_{i} / y\right)\right] \\
& \left.\frac{\partial g\left(\sigma_{1}^{2}, \sigma_{2}^{2}, \phi, \lambda\right)}{\partial \sigma_{2}^{2}}\right|_{\psi_{i}}=\frac{1}{2 h}\left[g\left(\sigma_{1 i}^{2}, \sigma_{2 i}^{2}+h, \phi, \lambda_{i} / y\right)-g\left(\sigma_{1 i}^{2}, \sigma_{2 i}^{2}-h, \phi, \lambda_{i} / y\right)\right], \\
& \left.\frac{\partial g\left(\sigma_{1}^{2}, \sigma_{2}^{2}, \phi, \lambda\right)}{\partial \lambda}\right|_{\psi_{i}}=\frac{1}{2 h}\left[g\left(\sigma_{1 i}^{2}, \sigma_{2 i}^{2}, \phi, \lambda_{i}+h / y\right)-g\left(\sigma_{1 i}^{2}, \sigma_{2 i}^{2}, \phi, \lambda_{i}+h / y\right)\right]
\end{aligned}
$$

com $h=10^{-2}$, e $\psi_{i}=\left(\sigma_{1 i}^{2}, \sigma_{2 i}^{2}, \phi, \lambda_{i}\right)$ é o vetor de parâmetros na i-ésima iteração.

A matriz de informação observada é constituída pelo elementos,

$$
I\left(\hat{\sigma}_{1}^{2}, \hat{\sigma}_{2}^{2}, \hat{\phi}, \hat{\lambda}\right)=\left[\begin{array}{lll}
I_{11} & I_{12} & I_{13} \\
& I_{22} & I_{23} \\
\operatorname{sim} . & & I_{33}
\end{array}\right],
$$

onde,

$$
\begin{aligned}
& I_{11}=-\left.\frac{\partial^{2} \ln L\left(\sigma_{1}^{2}, \sigma_{2}^{2}, \phi, \lambda / y\right)}{\partial\left(\sigma_{1}^{2}\right)^{2}}\right|_{\hat{\psi}}=-\frac{20}{\sigma_{1}^{2}}+\frac{46,10}{\left(\sigma_{1}^{2}\right)^{3}}-\left.\frac{\partial g\left(\sigma_{1}^{2}, \sigma_{2}^{2}, \phi, \lambda\right)}{\partial\left(\sigma_{1}^{2}\right)^{2}}\right|_{\hat{\psi}} \\
& I_{12}=-\left.\frac{\partial^{2} \ln L\left(\sigma_{1}^{2}, \sigma_{2}^{2}, \phi, \lambda / y\right)}{\partial \sigma_{1}^{2} \partial \sigma_{2}^{2}}\right|_{\hat{\psi}}=-\left.\frac{\partial^{2} g\left(\sigma_{1}^{2}, \sigma_{2}^{2}, \phi, \lambda\right)}{\partial \sigma_{1}^{2} \partial \sigma_{2}^{2}}\right|_{\hat{\psi}} \\
& I_{13}=-\left.\frac{\partial^{2} \ln L\left(\sigma_{1}^{2}, \sigma_{2}^{2}, \phi, \lambda / y\right)}{\partial \sigma_{1}^{2} \partial \lambda}\right|_{\hat{\psi}}=-\left.\frac{\partial^{2} g\left(\sigma_{1}^{2}, \sigma_{2}^{2}, \phi, \lambda\right)}{\partial \sigma_{1}^{2} \partial \lambda}\right|_{\hat{\psi}},
\end{aligned}
$$




$$
\begin{gathered}
I_{22}=-\left.\frac{\partial^{2} \ln L\left(\sigma_{1}^{2}, \sigma_{2}^{2}, \phi, \lambda / y\right)}{\partial\left(\sigma_{2}^{2}\right)^{2}}\right|_{\hat{\psi}}=-\left.\frac{\partial^{2} g\left(\sigma_{1}^{2}, \sigma_{2}^{2}, \phi, \lambda\right)}{\partial\left(\sigma_{2}^{2}\right)^{2}}\right|_{\hat{\psi}}, \\
I_{23}=-\left.\frac{\partial^{2} \ln L\left(\sigma_{1}^{2}, \sigma_{2}^{2}, \phi, \lambda / y\right)}{\partial \sigma_{2}^{2} \partial \lambda}\right|_{\hat{\psi}}=-\left.\frac{\partial^{2} g\left(\sigma_{1}^{2}, \sigma_{2}^{2}, \phi, \lambda\right)}{\partial \sigma_{2}^{2} \partial \lambda}\right|_{\hat{\psi}} \\
I_{33}=-\left.\frac{\partial^{2} \ln L\left(\sigma_{1}^{2}, \sigma_{2}^{2}, \phi, \lambda / y\right)}{\partial \lambda^{2}}\right|_{\hat{\psi}}=-\left.\frac{\partial^{2} g\left(\sigma_{1}^{2}, \sigma_{2}^{2}, \phi, \lambda\right)}{\partial \lambda^{2}}\right|_{\hat{\psi}} .
\end{gathered}
$$

Considerando a aproximação em (A.2), conseqüentemente temos,

$$
\begin{gathered}
\left.\frac{\partial^{2} g\left(\sigma_{1}^{2}, \sigma_{2}^{2}, \phi, \lambda\right)}{\partial\left(\sigma_{1}^{2}\right)^{2}}\right|_{\hat{\psi}}=\frac{1}{4 h^{2}}\left[g\left(\hat{\sigma}_{1}^{2}+2 h, \hat{\sigma}_{2}^{2}, \hat{\phi}, \hat{\lambda}\right)-2 g\left(\hat{\sigma}_{1}^{2}, \hat{\sigma}_{2}^{2}, \hat{\phi}, \hat{\lambda}\right)-g\left(\hat{\sigma}_{1}^{2}-2 h, \hat{\sigma}_{2}^{2}, \hat{\phi}, \hat{\lambda}\right)\right], \\
\begin{array}{c}
\left.\frac{\partial^{2} g\left(\sigma_{1}^{2}, \sigma_{2}^{2}, \phi, \lambda\right)}{\partial \sigma_{1}^{2} \partial \sigma_{2}^{2}}\right|_{\hat{\psi}}=\frac{1}{4 h^{2}}\left[g\left(\hat{\sigma}_{1}^{2}+h, \hat{\sigma}_{2}^{2}+h, \hat{\phi}, \hat{\lambda}\right)-g\left(\hat{\sigma}_{1}^{2}-h, \hat{\sigma}_{2}^{2}+h, \hat{\phi}, \hat{\lambda}\right)-\right. \\
\left.g\left(\hat{\sigma}_{1}^{2}+h, \hat{\sigma}_{2}^{2}-h, \hat{\phi}, \hat{\lambda}\right)-g\left(\hat{\sigma}_{1}^{2}-h, \hat{\sigma}_{2}^{2}-h, \hat{\phi}, \hat{\lambda}\right)\right]
\end{array}
\end{gathered}
$$

$$
\begin{array}{r}
\left.\frac{\partial^{2} g\left(\sigma_{1}^{2}, \sigma_{2}^{2}, \phi, \lambda\right)}{\partial \sigma_{1}^{2} \partial \lambda}\right|_{\hat{\psi}}=\frac{1}{4 h^{2}}\left[g\left(\hat{\sigma}_{1}^{2}+h, \hat{\sigma}_{2}^{2}, \hat{\phi}, \hat{\lambda}+h\right)-g\left(\hat{\sigma}_{1}^{2}-h, \hat{\sigma}_{2}^{2}, \hat{\phi}, \hat{\lambda}+h\right)-\right. \\
\left.g\left(\hat{\sigma}_{1}^{2}+h, \hat{\sigma}_{2}^{2}, \hat{\phi}, \hat{\lambda}-h\right)-g\left(\hat{\sigma}_{1}^{2}-h, \hat{\sigma}_{2}^{2}, \hat{\phi}, \hat{\lambda}-h\right)\right]
\end{array},
$$

$$
\left.\frac{\partial^{2} g\left(\sigma_{1}^{2}, \sigma_{2}^{2}, \phi, \lambda\right)}{\partial\left(\sigma_{2}^{2}\right)^{2}}\right|_{\hat{\psi}}=\frac{1}{4 h^{2}}\left[g\left(\hat{\sigma}_{1}^{2}, \hat{\sigma}_{2}^{2}+2 h, \hat{\phi}, \hat{\lambda}\right)-2 g\left(\hat{\sigma}_{1}^{2}, \hat{\sigma}_{2}^{2}, \hat{\phi}, \hat{\lambda}\right)-g\left(\hat{\sigma}_{1}^{2}, \hat{\sigma}_{2}^{2}-2 h, \hat{\phi}, \hat{\lambda}\right)\right],
$$




$$
\begin{aligned}
& \left.\frac{\partial^{2} g\left(\sigma_{1}^{2}, \sigma_{2}^{2}, \phi, \lambda\right)}{\partial \sigma_{2}^{2} \partial \lambda}\right|_{\hat{\psi}}=\frac{1}{4 h^{2}}\left[g\left(\hat{\sigma}_{1}^{2}, \hat{\sigma}_{2}^{2}+h, \hat{\phi}, \hat{\lambda}+h\right)-g\left(\hat{\sigma}_{1}^{2}, \hat{\sigma}_{2}^{2}-h, \hat{\phi}, \hat{\lambda}+h\right)-\right. \\
& \left.g\left(\hat{\sigma}_{1}^{2}, \hat{\sigma}_{2}^{2}+h, \hat{\phi}, \hat{\lambda}-h\right)-g\left(\hat{\sigma}_{1}^{2}, \hat{\sigma}_{2}^{2}-h, \hat{\phi}, \hat{\lambda}-h\right)\right] \\
& \left.\frac{\partial^{2} g\left(\sigma_{1}^{2}, \sigma_{2}^{2}, \phi, \lambda\right)}{\partial \lambda^{2}}\right|_{\hat{\psi}}=\frac{1}{4 h^{2}}\left[g\left(\hat{\sigma}_{1}^{2}, \hat{\sigma}_{2}^{2}, \hat{\phi}, \hat{\lambda}+2 h\right)-2 g\left(\hat{\sigma}_{1}^{2}, \hat{\sigma}_{2}^{2}, \hat{\phi}, \hat{\lambda}\right)-g\left(\hat{\sigma}_{1}^{2}, \hat{\sigma}_{2}^{2}, \hat{\phi}, \hat{\lambda}-2 h\right)\right]
\end{aligned}
$$

Também obtemos as matrizes Hessianas nas aproximações de Laplace de modo semelhante. 


\section{Apêndice B}

\section{Obtenção de Regiões HPD}

Em inferência Bayesiana, em geral, existe o interesse em encontrar uma região $\mathrm{R}$, do espaço paramétrico, para a qual a probabilidade de conter a densidade a posteriori dos parâmetros $\left(\theta_{1}, \theta_{2}\right)$ é $(1-\alpha)$. Tal região $(R)$ é chamada de região a "Posteriori de Maior Probabilidade " (HPD) se,

$$
\begin{aligned}
& \int_{R} \pi\left(\theta_{1}, \theta_{2} / y\right)=1-\alpha, \quad \text { e se } \\
& \pi\left(\theta_{1}, \theta_{2} / y\right) \geq \pi\left(\theta_{1}^{\prime}, \theta_{2}^{\prime} / y\right) \text { para todo } \operatorname{par}\left(\theta_{1}, \theta_{2}\right) \in R \text { e }\left(\theta_{1}^{\prime}, \theta_{2}^{\prime}\right) \notin R .
\end{aligned}
$$

onde $\pi\left(\theta_{1}, \theta_{2} / y\right)$ é a densidade a posteriori conjunta para $\left(\theta_{1}, \theta_{2}\right)$ (ver por exemplo, Box e Tiao, 1973).

Nesse trabalho, em especial, avaliamos os contornos dessas regiões, com o objetivo de investigar a aproximação normal das densidade a posteriori dos parâmetros.

Em problemas práticos, a obtenção dessas regiões pode apresentar dificuldades. Porém, podemos usar o fato que, para grandes amostras a distribuição conjunta tende a normalidade e, portanto, temos que a quantidade,

$$
-2 \ln \left\{\frac{\pi\left(\theta_{1}, \theta_{2} / y\right)}{\pi\left(\tilde{\theta}_{1}, \tilde{\theta}_{2} / y\right)}\right\},
$$


é aproximadamente distribuída como uma Qui-Quadrado com 2 graus de liberdade, onde $\pi\left(\tilde{\theta}_{1}, \tilde{\theta}_{2} / y\right)$ é a densidades a posteriori conjunta calculada na moda $\left(\tilde{\theta}_{1}, \tilde{\theta}_{2}\right)$.

Portando, os contornos constituídos por todos os valores de $\left(\theta_{1}, \theta_{2}\right)$ que satisfazem a relação

$$
\ln \pi\left(\theta_{1}, \theta_{2} / y\right)-\log \pi\left(\tilde{\theta}_{1}, \tilde{\theta}_{2} / y\right)+\frac{\chi_{2(1-\alpha)}^{2}}{2}=0
$$

onde $\chi_{2(1-\alpha)}^{2}$ é o quantil da distribuição Qui-Quadrado dado por

$$
P\left(\chi_{2}^{2} \leq \chi_{2(1-\alpha)}^{2}\right)=1-\alpha,
$$

incluem uma região (HPD) com $(1-\alpha)$ de probabilidade.

Para esse trabalho em particular obtemos os contornos de regiões HPD para as densidades a posteriori marginais (3.10) e (3.11).

Considerando a densidade a posteriori marginal conjunta (3.10) e assumindo um nível de 0,95 de probabilidade, e portanto $\chi_{2,0,95}^{2}=5,9915$, com $\tilde{\sigma}_{1}^{2}=v_{1} m_{1} /\left(v_{1}+2\right)$ e $\tilde{\sigma}_{2}^{2}=\left\{v_{2} m_{2} /\left(v_{2}+2\right)-\tilde{\sigma}_{1}^{2}\right\} / K$, temos:

$$
\pi\left(\tilde{\sigma}_{1}^{2}, \tilde{\sigma}_{2}^{2} / y\right) \propto\left(\frac{v_{1} m_{1}}{v_{1}+2}\right)^{-\left(\frac{v_{1}}{2}+1\right)}\left(\frac{v_{2} m_{2}}{v_{2}+2}\right)^{-\left(\frac{v_{2}}{2}+1\right)} \exp \left\{-\frac{\left(v_{1}+v_{2}+4\right)}{2}\right\} .
$$

Os contornos que incluem uma região HPD com 95\% de probabilidade são obtidos pelos valores de $\left(\sigma_{1}^{2}, \sigma_{2}^{2}\right)$ que satisfazem a equação: 


$$
\ln \pi\left(\sigma_{1}^{2}, \sigma_{2}^{2} / y\right)+\left(\frac{v_{1}}{2}+1\right) \ln \left(\frac{v_{1} m_{1}}{v_{1}+2}\right)+\left(\frac{v_{2}}{2}+1\right) \ln \left(\frac{v_{2} m_{2}}{v_{2}+2}\right)-\frac{\left(v_{1}+v_{2}+4\right)}{2}-2,996=0 .
$$

Para obter tais pontos, fixamos o valor de um dos parâmetros, $\sigma_{1}^{2}$ por exemplo, e encontramos o valor do outro parâmetro através do método iterativo de NewtonRaphson. A conversão do algoritmo ocorrerá se o valor do parâmetro que foi fixado é um ponto pertencente ao contorno. 


\section{Apêndice C}

\section{Expressões Utilizadas no Cálculo do Gráfico T-plot}

Para avaliar a normalidade das densidades a posteriori marginais conjunta para $\left(\sigma_{1}^{2}, \sigma_{2}^{2}\right)$ (3.10) e (3.11) utilizando o T-plot de Hills e Smith podemos ter dificuldades uma vez que temos dois parâmetros no modelo. Para simplificar esse problema, consideramos, como alternativa, as densidades a posteriori "profile" para cada parâmetro. A densidade a posteriori "profile" para $\sigma_{1}^{2}$ é dada por $\pi_{\hat{\sigma}_{2}^{2}}\left(\sigma_{1}^{2} / y\right)=\pi\left(\sigma_{1}^{2}, \hat{\sigma}_{2}^{2} / y\right)$, onde $\hat{\sigma}_{2}^{2}$ maximiza a densidade a posteriori marginal conjunta $\pi\left(\sigma_{1}^{2}, \sigma_{2}^{2} / y\right)$ para cada valor de $\sigma_{1}^{2}$. Da mesma forma, a densidade a posteriori "profile" para $\sigma_{2}^{2}$ é dada por $\pi_{\hat{\sigma}_{1}^{2}}\left(\sigma_{2}^{2} / y\right)=\pi\left(\hat{\sigma}_{1}^{2}, \sigma_{2}^{2} / y\right)$, onde $\hat{\sigma}_{1}^{2}$ maximiza $\pi\left(\sigma_{1}^{2}, \sigma_{2}^{2} / y\right)$ para cada valor de $\sigma_{2}^{2}$.

Considerando a densidade a posteriori marginal conjunta (3.11) o logaritmo da densidade a posteriori "profile" para $\sigma_{1}^{2}$ é dada por

$\ln \pi_{\hat{\sigma}_{2}^{2}}\left(\sigma_{1}^{2} / y\right) \propto-\frac{\left(v_{1}+2\right)}{2} \ln \left(\sigma_{1}^{2}\right)-\frac{\left(v_{2}+2\right)}{2} \ln \left(\sigma_{1}^{2}+K \hat{\sigma}_{2}^{2}\right)-\frac{1}{2}\left[\frac{v_{1} m_{1}}{\sigma_{1}^{2}}+\frac{v_{2} m_{2}}{\sigma_{1}^{2}+K \hat{\sigma}_{2}^{2}}\right]$,

onde, para cada valor de $\sigma_{1}^{2}, \hat{\sigma}_{2}^{2}$ é obtido de,

$$
\hat{\sigma}_{2}^{2}=\left(\frac{v_{2} m_{2}}{v_{2}+2}-\sigma_{1}^{2}\right) / K
$$

O T-plot de Hills e Smith é obtido ponto a ponto a partir da relação, 


$$
T\left(\sigma_{1}^{2}\right)=\operatorname{sgn}\left(\sigma_{1}^{2}-\tilde{\sigma}_{1}^{2}\right)\left[-2 \ln \pi_{\hat{\sigma}_{2}^{2}}\left(\sigma_{1}^{2} / y\right)+2 \ln \pi\left(\tilde{\sigma}_{1}^{2}, \tilde{\sigma}_{2}^{2} / y\right)\right]^{1 / 2},
$$

onde,

$$
\tilde{\sigma}_{1}^{2}=\frac{v_{1} m_{1}}{v_{1}+2} \text { e } \quad \tilde{\sigma}_{2}^{2}=\left(\frac{v_{2} m_{2}}{v_{2}+2}-\frac{v_{1} m_{1}}{v_{1}+2}\right) / K
$$

Também, considerando a densidade a posteriori marginal conjunta (3.11), o logaritmo da densidade a posteriori "profile" para $\sigma_{2}^{2}$ é dado por

$$
\ln \pi_{\hat{\sigma}_{1}^{2}}\left(\sigma_{2}^{2} / y\right) \propto-\frac{\left(v_{1}+2\right)}{2} \ln \left(\hat{\sigma}_{1}^{2}\right)-\frac{\left(v_{2}+2\right)}{2} \ln \left(\hat{\sigma}_{1}^{2}+K \sigma_{2}^{2}\right)-\frac{1}{2}\left[\frac{v_{1} m_{1}}{\hat{\sigma}_{1}^{2}}+\frac{v_{2} m_{2}}{\hat{\sigma}_{1}^{2}+K \sigma_{2}^{2}}\right],
$$

onde, para cada valor de $\sigma_{2}^{2}, \hat{\sigma}_{1}^{2}$ é obtido da relação,

$$
-\frac{\left(v_{1}+2\right)}{2 \hat{\sigma}_{1}^{2}}-\frac{\left(v_{2}+2\right)}{2\left(\hat{\sigma}_{1}^{2}+K \sigma_{2}^{2}\right)}+\frac{1}{2}\left[\frac{v_{1} m_{1}}{\hat{\sigma}_{1}^{2}}+\frac{v_{2} m_{2}}{\hat{\sigma}_{1}^{2}+K \sigma_{2}^{2}}\right]=0
$$

O T-plot de Hills e Smith para $\sigma_{2}^{2}$ é obtido da relação,

$$
T\left(\sigma_{2}^{2}\right)=\operatorname{sgn}\left(\sigma_{2}^{2}-\tilde{\sigma}_{2}^{2}\right)\left[-2 \ln \pi_{\hat{\sigma}_{1}^{2}}\left(\sigma_{2}^{2} / y\right)+2 \ln \pi\left(\tilde{\sigma}_{1}^{2}, \tilde{\sigma}_{2}^{2} / y\right)\right]^{1 / 2},
$$

$\operatorname{com}\left(\tilde{\sigma}_{1}^{2}, \tilde{\sigma}_{2}^{2}\right)$ dados em (C.4). 


\section{Apêndice D}

\section{Alguns Programas Desenvolvidos}

A seguir apresentamos alguns programas desenvolvidos os quais foram utilizados para a análise dos dados.

\section{PROGRAMA 1}

Programa para calcular o contorno de da região HPD da densidade a posteriori conjunta para $\left(\sigma_{1}^{2}, \sigma_{2}^{2}\right)$. Como exemplo, estamos supondo que temos o conjunto de dados do exemplo 2, a densidade a posteriori (3.11) e um nível de credibilidade de $95 \%$. O programa foi editado em linguagem compatível ao software MINITAB. O programa calcula o valor de $\sigma_{1}^{2}$ que satisfaz a equação (B.4), através do algoritmo de Newton Raphson, para cada valor de $\sigma_{2}^{2}$. Os valores de $\sigma_{1}^{2}$ e $\sigma_{2}^{2}$ que pertencem ao contorno da região HPD serão armazenados na colunas $\mathrm{C} 4$ e $\mathrm{C} 5$ respectivamente.
Let $\mathrm{k} 30=1$
\{contador
Let $\mathrm{k} 40=1$
\{contador
Let $\mathrm{k} 10=13$
$\left\{\right.$ valor inicial de $\left.\sigma_{1}^{2}\right\}$
Let $\mathrm{k} 11=3.6$
$\left\{\right.$ valor inicial de $\left.\sigma_{2}^{2}\right\}$

Store 'Nr'

Noecho

\{início da rotina de Newton Raphson para obter $\sigma_{1}^{2}$ \}

Let $\mathrm{C} 1(\mathrm{k} 30)=26 * \log \mathrm{e}(\mathrm{k} 10)+7 * \log (\mathrm{k} 10+5 * \mathrm{k} 11)+358.7014 / \mathrm{k} 10+41.6816 /(\mathrm{k} 10+5 * \mathrm{k} 11)$

- $113.7233-5.9915$

Let $\mathrm{C} 2(\mathrm{k} 30)=26 / \mathrm{k} 10+7 /\left(\mathrm{k} 10+5^{*} \mathrm{k} 11\right)-358.7014 /\left(\mathrm{k} 10^{* *} 2\right)-41.6816 /((\mathrm{k} 10+5 * \mathrm{k} 11) * * 2)$

Let $\mathrm{C} 3(\mathrm{k} 30)=\mathrm{k} 10-\mathrm{C} 1(\mathrm{k} 30) / \mathrm{c} 2(\mathrm{k} 30)$

Let $\mathrm{k} 10=\mathrm{C} 3(\mathrm{k} 30)$

Let $\mathrm{k} 30=\mathrm{k} 30+1$

End

\{final da rotina $\}$ 
Store 'Cont'

Exec 'Nr' 15

Let $\mathrm{C} 4(\mathrm{k} 40)=\mathrm{k} 1$

Let $\mathrm{C} 5(\mathrm{k} 40)=\mathrm{k} 11$

Let $\mathrm{C} 6(\mathrm{k} 40)=\mathrm{C} 1(\mathrm{k} 30-1)$

Let $\mathrm{k} 11=\mathrm{k} 11-0.2$

Let $\mathrm{k} 40=\mathrm{k} 40+1$

Let $\mathrm{k} 30=1$

Let $\mathrm{k} 10=13$

End

Exec 'Cont'
$\{$ início da rotina que sucessivamente executa o algoritmo Newton Raphson, incrementa o valor de $\sigma_{2}^{2}$ e obtém novos valores de $\left.\sigma_{1}^{2}\right\}$

\{final da rotina

\{comando para executar o programa\} 


\section{PROGRAMA 2}

Programa para obter os estimadores de máxima verossimilhança do modelo com mistura de distribuições de normais. O programa é executável no módulo IML do SAS. O programa segue a seguinte rotina: i) para cada valor de $\phi(\phi=-1,0,1)$ são calculadas numericamente, as derivadas parciais numéricas do logaritmo da função de verossimilhança (6.1) em relação a $\sigma_{1}^{2}, \sigma_{2}^{2}$ e $\lambda$; ii) através do método iterativo de Newton-Raphson são obtido os valores de $\sigma_{1}^{2}, \sigma_{2}^{2}$ e $\lambda$ que maximizam o logaritmo da função de verossimilhança; iii) é obtida a matriz de derivadas de segunda ordem (numericamente) nos máximos; iv) para cada valor $\phi$, é imprimido: o número de iterações necessárias até a convergência do algoritmo (IT), os valores máximos obtidos (PAR'), o logaritmo de função de verossimilhança (LNL), o máximo (em valor absoluto) do erro cometido até a convergência (ERRO) e a matriz de segundas derivadas, a menos do sinal (MINF), (observar saída).

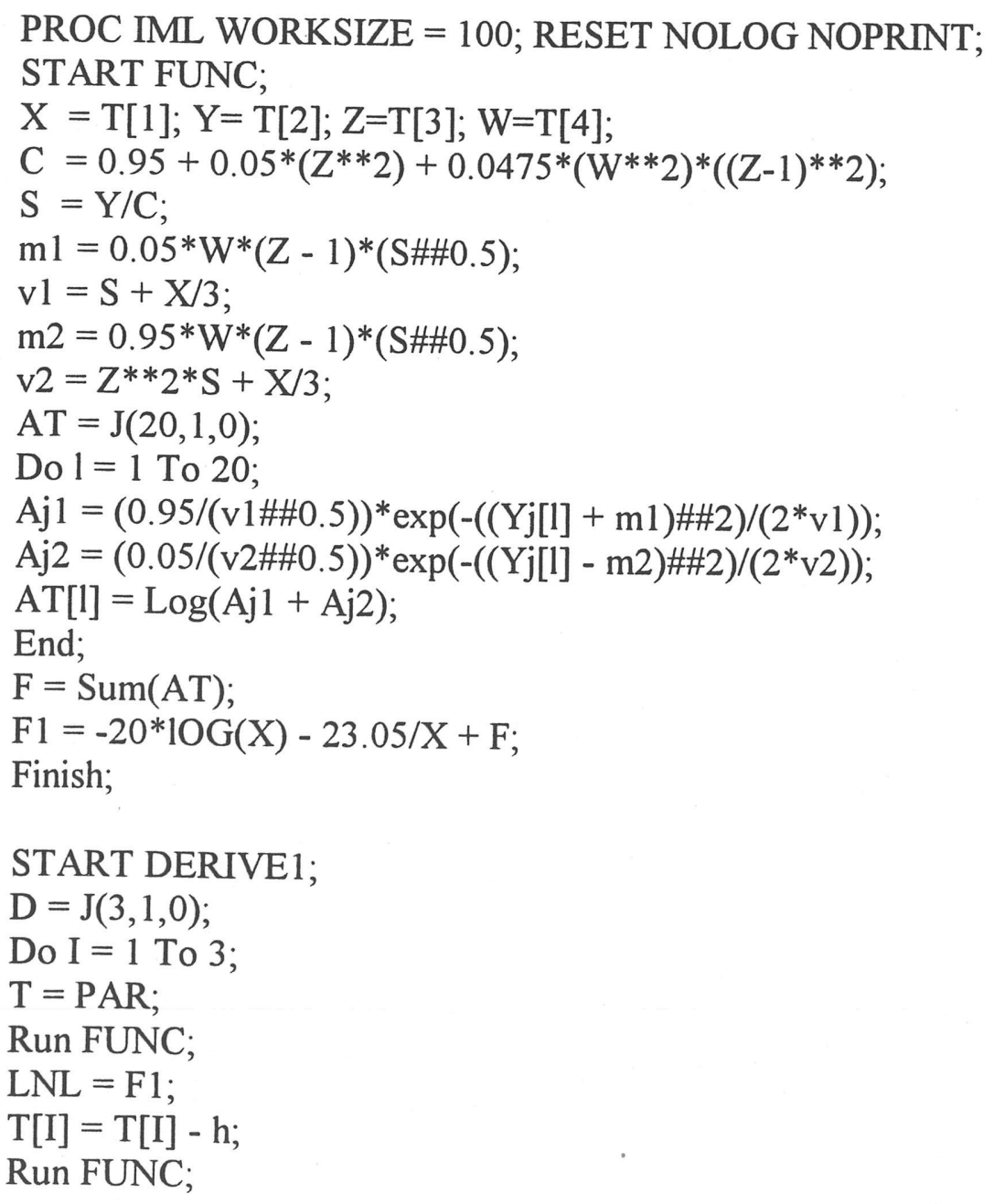


$\mathrm{D} 1=\mathrm{F}$;

$\mathrm{T}=\mathrm{PAR}$;

$\mathrm{T}[\mathrm{I}]=\mathrm{T}[\mathrm{I}]+\mathrm{h}$;

Run FUNC;

$\mathrm{D} 2=\mathrm{F}$;

$\mathrm{D}[\mathrm{I}]=(\mathrm{D} 2-\mathrm{D} 1) /(2 * \mathrm{~h})$;

IF I = 1 THEN DO; D[I] = D[I] - 20/PAR[1] + 23.05/(PAR[1]\#\#2);

End;

End;

Finish;

START DERIVE2;

$\mathrm{M}=\mathrm{J}(3,3,0)$;

Do $\mathrm{K}=1$ To 3 ;

Do $\mathrm{J}=1$ To 3;

$\mathrm{A} 1=\mathrm{PAR} ; \mathrm{A} 2$ = PAR; B1=PAR; B2=PAR;

$\mathrm{A} 1[\mathrm{~K}]=\mathrm{PAR}[\mathrm{K}]+\mathrm{h}$;

$\mathrm{A} 1[\mathrm{~J}]=\mathrm{A} 1[\mathrm{~J}]+\mathrm{h}$

$\mathrm{T}=\mathrm{A} 1$;

Run FUNC;

$\mathrm{AUX1}=\mathrm{F}$;

$\mathrm{A} 2[\mathrm{~K}]=\mathrm{PAR}[\mathrm{K}]-\mathrm{h}$;

$\mathrm{A} 2[\mathrm{~J}]=\mathrm{A} 2[\mathrm{~J}]-\mathrm{h}$;

$\mathrm{T}=\mathrm{A} 2$;

Run FUNC;

$\mathrm{AUX} 2=\mathrm{F}$;

$\mathrm{B} 1[\mathrm{~K}]=\mathrm{PAR}[\mathrm{K}]+\mathrm{h}$;

$\mathrm{C} 1=\mathrm{B} 1$;

$\mathrm{C} 1[\mathrm{~J}]=\mathrm{B} 1[\mathrm{~J}]-\mathrm{h}$

$\mathrm{T}=\mathrm{C} 1$;

Run FUNC;

Aux3 = F;

$\mathrm{B} 2[\mathrm{~K}]=\mathrm{PAR}[\mathrm{K}]-\mathrm{h}$;

$\mathrm{C} 2=\mathrm{B} 2$;

$\mathrm{C} 2[\mathrm{~J}]=\mathrm{B} 2[\mathrm{~J}]+\mathrm{h}$;

$\mathrm{T}=\mathrm{C} 2$;

Run FUNC;

$\mathrm{AUX} 4=\mathrm{F}$;

$\mathrm{M}[\mathrm{K}, \mathrm{J}]=(\mathrm{AUX} 1-\mathrm{AUX} 3-\mathrm{AUX} 4+\mathrm{AUX} 2) /\left(4^{*}\left(\mathrm{~h}^{* * 2}\right)\right)$;

IF $(\mathrm{K}=1)$ \& $(\mathrm{J}=1)$ THEN DO;

$\mathrm{M}[\mathrm{K}, \mathrm{J}]=\mathrm{M}[\mathrm{K}, \mathrm{J}]+20 /\left(\operatorname{PAR}[1]^{* *} 2\right)-46.1 /(\operatorname{PAR}[1] \# \# 3) ; \mathrm{END} ;$

End;

End;

Finish;

START NEWTON;

Run DERIVE1; 
Do IT $=1$ To 10

While (Max(Abs(D) > 1E-10));

Run DERIVE2;

DELTA = - SOLVE(M,D);

$\mathrm{AA}=\operatorname{PAR}\left[\left\{\begin{array}{lll}1 & 2 & 3\end{array}\right\}\right]$

$\mathrm{AA}=\mathrm{AA}+\mathrm{DELTA}$;

PAR = AA/PAR[4];

Run DERIVE1;

End;

Finish;

$\mathrm{Yj}=\{-3.682,-2.057,-1.780,-1.238,-0.797,-0.671,-0.646,-0.471,-0.436,-0.401$, $-0.378,0,0.112,0.791,0.923,1.571,1.712,4.223,6.415,7.072\}$;

$\mathrm{h}=1 \mathrm{E}-2$;

$\operatorname{PAR}=\{1,4,4,-1\}$

START TUDO;

Do $\mathrm{O}=1$ To 3 ;

Run NEWTON;

Run DERIVE2;

$\mathrm{MINF}=-1 * \mathrm{M}$;

$\mathrm{ERRO}=\operatorname{Max}(\operatorname{Abs}(\mathrm{D}))$;

Print IT (PAR');

Print LNL ERRO;

Print MINF;

$\operatorname{PAR}[1]=1 ; \operatorname{PAR}[2]=4 ; \operatorname{PAR}[3]=4 ;$

$\operatorname{PAR}[4]=\operatorname{PAR}[4]+1$

End;

Finish;

Run TUDO; 


\section{Saída do programa 2}

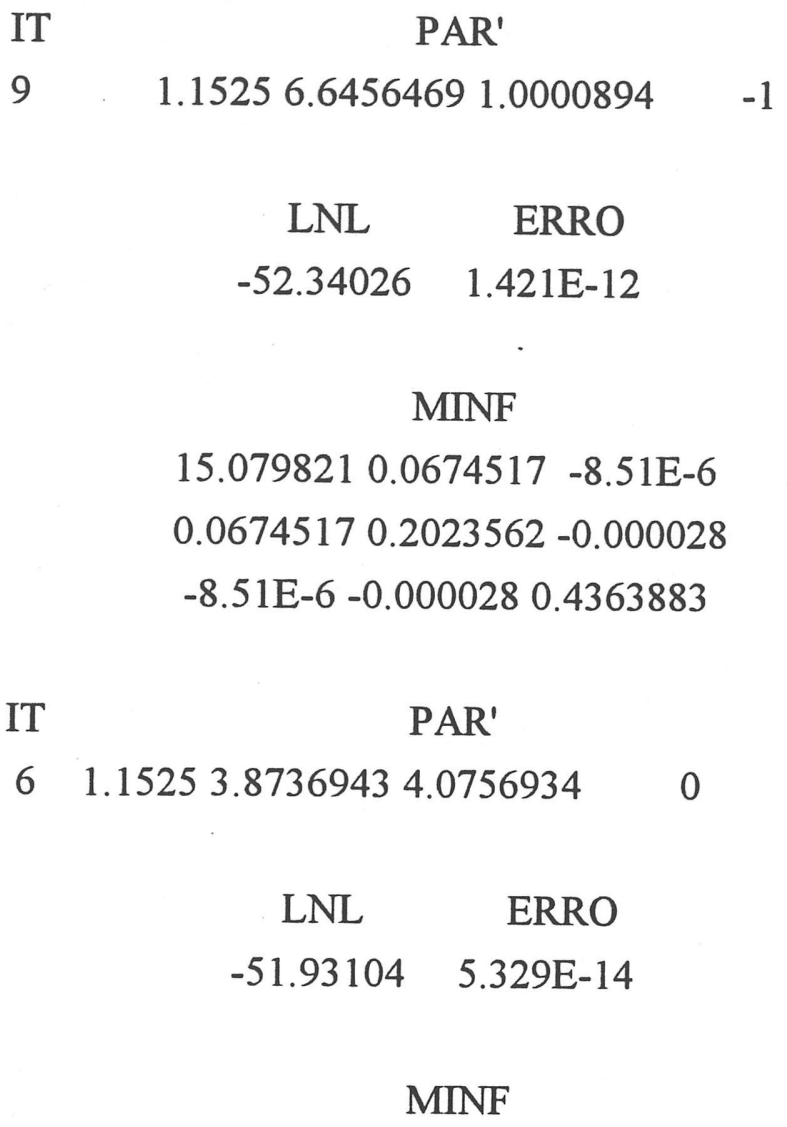

$15.1171820 .0982705-0.092311$

$0.09827050 .2320543-0.071286$

$-0.092311-0.0712860 .2336015$

IT PAR' $^{\prime}$

$71.15464243 .13549493 .7625441 \quad 1$

$$
\begin{aligned}
& \text { LNL ERRO } \\
& -50.41669 \text { 2.149E-11 } \\
& \text { MINF } \\
& 15.0560130 .1620568-0.156551 \\
& 0.16205680 .3836591-0.060894 \\
& -0.156551-0.0608940 .3757428
\end{aligned}
$$




\section{PROGRAMA 3}

Programa para calcular a densidade a posteriori marginal aproximada para $\lambda$ (5.10), dado $\phi=1$, para as diferentes densidades a priori $\pi_{i}\left(\sigma_{1}^{2}, \sigma_{2}^{2}, \lambda / \phi\right.$ ) (observar figura (6.17)). Programa elaborado em linguagem IML do software SAS. Como no programa 2, é utilizado derivação numérica para calcular os valores $\hat{\sigma}_{1}^{2}$ e $\hat{\sigma}_{2}^{2}$ que maximizam $-n h_{\lambda}\left(\sigma_{1}^{2}, \sigma_{2}^{2}\right)$ cada valor de $\lambda$, através do método de Newton-Raphson, e para obter a matriz Hessiana de $h_{\lambda}\left(\hat{\sigma}_{1}^{2}, \hat{\sigma}_{2}^{2}\right)$.

PROC IML WORKSIZE = 100; RESET NOLOG NONAME NOPRINT;

Start FUNC(T,N,F,F1);

$\mathrm{X}=\mathrm{T}[1] ; \mathrm{Y}=\mathrm{T}[2] ; \mathrm{Z}=\mathrm{T}[3] ; \mathrm{W}=\mathrm{T}[4]$;

$\mathrm{C}=0.95+0.05 *\left(\mathrm{Z}^{* * 2}\right)+0.0475^{*}\left(\mathrm{~W}^{* *} 2\right)^{*}\left((\mathrm{Z}-1)^{* *} 2\right)$;

$\mathrm{S}=\mathrm{Y} / \mathrm{C}$

$\mathrm{ml}=0.05 * \mathrm{~W} *(\mathrm{Z}-1) *(\mathrm{~S} \# 0.5)$;

$\mathrm{v} 1=\mathrm{S}+\mathrm{X} / 3$;

$\mathrm{m} 2=0.95 * \mathrm{~W}^{*}(\mathrm{Z}-1) *(\mathrm{~S} \# 0.5)$;

$\mathrm{v} 2=\mathrm{Z} * * 2 * \mathrm{~S}+\mathrm{X} / 3$;

$\mathrm{AT}=\mathrm{J}(20,1,0)$;

Do $1=1$ To 20 ;

$\mathrm{Aj} 1=(0.95 /(\mathrm{vl} \# \# 0.5)) * \exp (-((\mathrm{Yj}[1]+\mathrm{m} 1) \# \# 2) /(2 * \mathrm{v} 1))$;

$\mathrm{Aj} 2=(0.05 /(\mathrm{v} 2 \# \# 0.5)) * \exp (-((\mathrm{Yj}[1]-\mathrm{m} 2) \# \# 2) /(2 * \mathrm{v} 2)) ;$

$\operatorname{AT}[1]=\log (\operatorname{Aj} 1+\operatorname{Aj} 2)$;

End;

$\mathrm{F}=\operatorname{Sum}(\mathrm{AT})$;

$\mathrm{F} 1=-20 * \log (\mathrm{X})-23.05 / \mathrm{X}+\mathrm{F}$;

Finish;

Start DERIVE1;

$\mathrm{D}=\mathrm{J}(2,1,0)$;

DO I = 1 TO 2;

$\mathrm{AX1}=\mathrm{PAR}$

Run FUNC(AX1, Yj,F,F1);

$\mathrm{LNL}=\mathrm{F} 1$;

$\mathrm{AX} 1[\mathrm{I}]=\mathrm{AX} 1[\mathrm{I}]-\mathrm{h}$;

$\mathrm{AX} 2=\mathrm{PAR}$

$\mathrm{AX} 2[\mathrm{I}]=\mathrm{AX} 2[\mathrm{I}]+\mathrm{h}$;

Run FUNC(AX1,Yj,F,F1);

$\mathrm{D} 1=\mathrm{F}$;

Run FUNC(AX2,Yj,F,F1);

$\mathrm{D} 2=\mathrm{F}$;

$\mathrm{D}[\mathrm{I}]=(\mathrm{D} 2-\mathrm{D} 1) /(2 * \mathrm{~h})$;

IF I = 1 THEN DO; D[I] = D[I] - 20/PAR[1] + 23.05/(PAR[1]\#\#2);

End;

End;

Finish; 
Start DERIVE2;

$\mathrm{M}=\mathrm{J}(2,2,0)$;

DO K = 1 TO 2;

DO J = 1 TO 2;

$\mathrm{A} 1=\mathrm{PAR} ; \mathrm{A} 2=\mathrm{PAR} ; \mathrm{B} 1=\mathrm{PAR} ; \mathrm{B} 2=\mathrm{PAR} ;$

$\mathrm{A} 1[\mathrm{~K}]=\mathrm{PAR}[\mathrm{K}]+\mathrm{h}$;

$\mathrm{A} 1[\mathrm{~J}]=\mathrm{A} 1[\mathrm{~J}]+\mathrm{h}$;

$\mathrm{A} 2[\mathrm{~K}]=\mathrm{PAR}[\mathrm{K}]-\mathrm{h}$;

$\mathrm{A} 2[\mathrm{~J}]=\mathrm{A} 2[\mathrm{~J}]-\mathrm{h}$;

$\mathrm{B} 1[\mathrm{~K}]=\mathrm{PAR}[\mathrm{K}]+\mathrm{h}$;

$\mathrm{C} 1=\mathrm{B} 1$;

$\mathrm{C} 1[\mathrm{~J}]=\mathrm{B} 1[\mathrm{~J}]-\mathrm{h}$;

$\mathrm{B} 2[\mathrm{~K}]=\mathrm{PAR}[\mathrm{K}]-\mathrm{h}$;

$\mathrm{C} 2=\mathrm{B} 2$;

$\mathrm{C} 2[\mathrm{~J}]=\mathrm{B} 2[\mathrm{~J}]+\mathrm{h}$;

Run FUNC(A1, Yj,F,F1);

$\mathrm{AUX} 1=\mathrm{F}$;

Run FUNC(A2, Yj,F,F1);

$\mathrm{AUX} 2=\mathrm{F}$;

Run FUNC(C1, Yj,F,F1);

AUX3 = F;

Run FUNC(C2,Yj,F,F1);

$\mathrm{AUX} 4=\mathrm{F}$;

$\mathrm{M}[\mathrm{K}, \mathrm{J}]=(\mathrm{AUX} 1-\mathrm{AUX} 3-\mathrm{AUX} 4+\mathrm{AUX} 2) /\left(4^{*}\left(\mathrm{~h}^{* *} 2\right)\right)$;

IF $(K=1) \&(\mathrm{~J}=1)$ THEN DO;

$\mathrm{M}[\mathrm{K}, \mathrm{J}]=\mathrm{M}[\mathrm{K}, \mathrm{J}]+20 /(\operatorname{PAR}[1] \# \# 2)-46.1 /(\operatorname{PAR}[1] \# \# 3) ;$ End;

End;

End;

Finish;

Start NEWTON;

Run DERIVE1;

DO IT = 1 TO 20

While $(\operatorname{Max}(\operatorname{Abs}(\mathrm{D})>1 \mathrm{E}-10))$;

Run DERIVE2;

DELTA = - SOLVE(M,D);

$\mathrm{AA}=\operatorname{PAR}\left[\left\{\begin{array}{ll}1 & 2\end{array}\right\}\right]$;

$\mathrm{AA}=\mathrm{AA}+\mathrm{DELTA}$;

$\mathrm{PAR}=\mathrm{AA} / / \mathrm{PAR}[3] / / \mathrm{PAR}[4]$;

Run DERIVE1;

End;

Finish;

$\mathrm{Yj}=\{-3.682,-2.057,-1.780,-1.238,-0.797,-0.671,-0.646,-0.471,-0.436,-0.401$, $-0.378,0,0.112,0.791,0.923,1.571,1.712,4.223,6.415,7.072\}$;

$\mathrm{h}=1 \mathrm{E}-2$;

$\operatorname{PAR}=\{1,5,1,1\}$ 
Start POSTER;

DO N = 1 TO 3;

Run NEWTON;

Run DERIVE2;

$\mathrm{DT}=\operatorname{DET}(-\mathrm{M})$;

POS1 = Exp(LNL-Log(PAR[3]) - Log(PAR[1]) - Log( PAR[1] + 3*PAR[2] ) -

$0.5 * \mathrm{LOG}(\mathrm{DT})+53)$;

POS2 = Exp(LNL-((PAR[3]-3)**2)/8- Log(PAR [1]) - Log(PAR[1] + 3*PAR[2]) -

$0.5 *$ LOG(DT)+53);

POS3 $=\operatorname{Exp}(\operatorname{LNL}-((\operatorname{PAR}[3]-3) * * 2) / 2-\log (\operatorname{PAR}[1])-\log (\operatorname{PAR}[1]+3 *$ PAR [2] $)-$

$0.5 *$ LOG(DT)+53);

$\mathrm{ERRO}=\operatorname{Max}(\mathrm{Abs}(\mathrm{D}))$;

PINTER = PAR[1]//PAR[2]//PAR[3];

Print (PINTER') POS1 POS2 POS3 ERRO;

$\operatorname{PAR}[3]=\operatorname{PAR}[3]+0.1$;

End;

Finish;

Run POSTER; 